\title{
Insulin/insulin-like growth factor signaling in $C$. elegans
}

Coleen T. Murphy ${ }^{1 \S}$ and Patrick J. Hu ${ }^{2 \S}$

${ }^{1}$ Lewis-Sigler Institute for Integrative Genomics and Department of Molecular Biology, Princeton University, Princeton, NJ 08544 USA

${ }^{2}$ Institute of Gerontology and Departments of Internal Medicine and Cell and Developmental Biology, University of Michigan Medical School, Ann Arbor, MI 48109 USA

\section{Table of Contents}

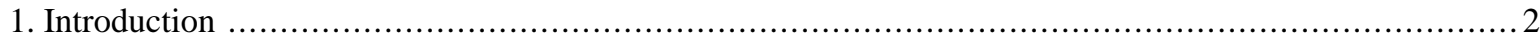

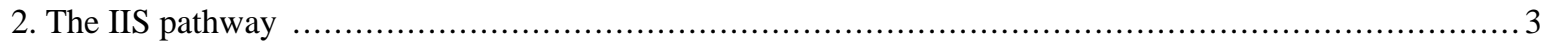

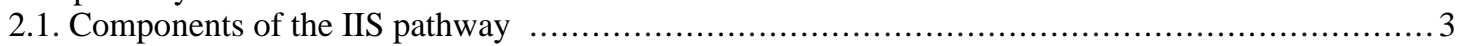

2.2. Discovery of the $C$. elegans IIS pathway ...................................................

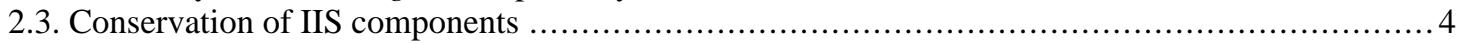

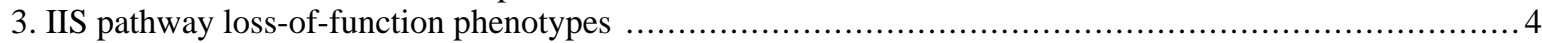

3.1. IIS regulation of development: dauer, L1 arrest, and germline proliferation .......................5

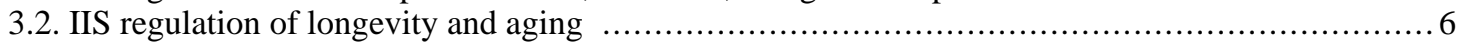

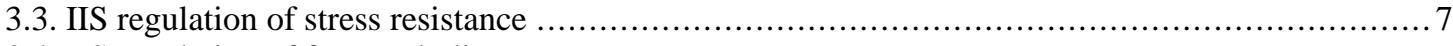

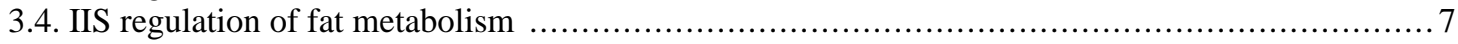

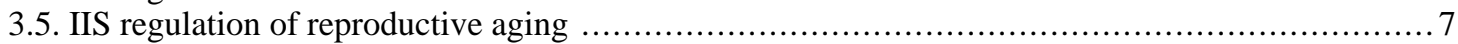

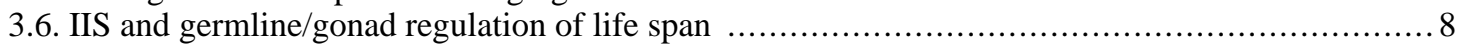

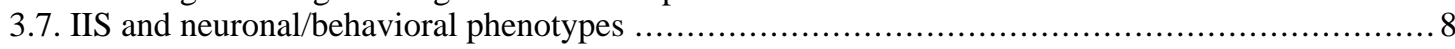

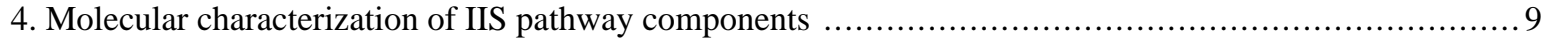

4.1. DAF-2, the $C$. elegans insulin/IGF-1 receptor ortholog ........................................

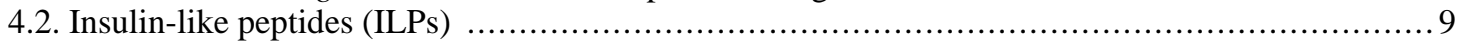

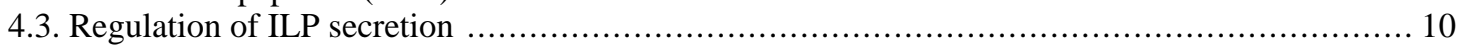

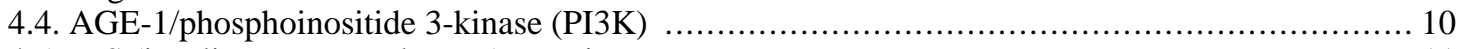

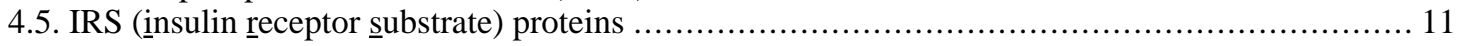

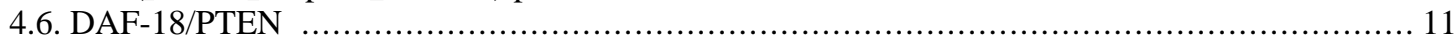

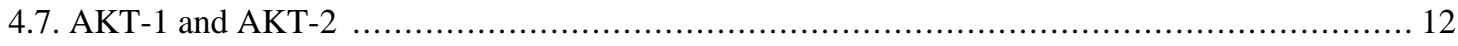

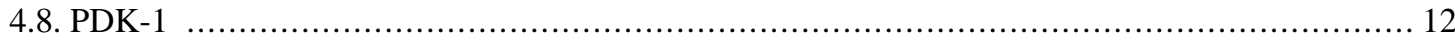

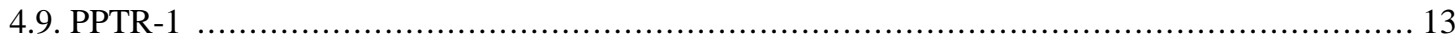

${ }^{*}$ Edited by Iva Greenwald Last revised July 11, 2013, Published December 26, 2013. This chapter should be cited as: Murphy C.T., Hu P.J. Insulin/insulin-like growth factor signaling in C. elegans (December 26, 2013), WormBook, ed. The C. elegans Research Community, WormBook, doi/10.1895/wormbook.1.164.1, http://www.wormbook.org.

Copyright: () 2013 Coleen T. Murphy and Patrick J. Hu. This is an open-access article distributed under the terms of the Creative Commons Attribution License, which permits unrestricted use, distribution, and reproduction in any medium, provided the original author and source are credited.

${ }^{\S}$ To whom correspondence should be addressed. E-mail: ctmurphy@princeton.edu; pathu@umich.edu 
4.10. SGK-1 …

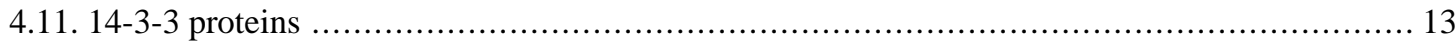

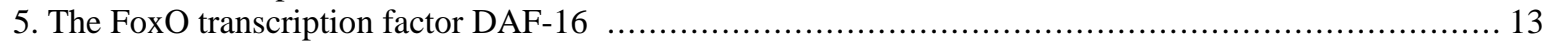

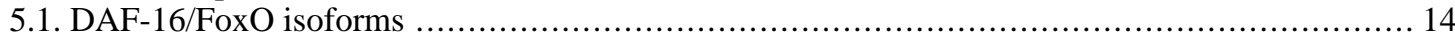

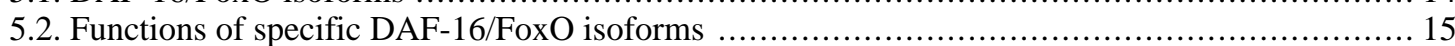

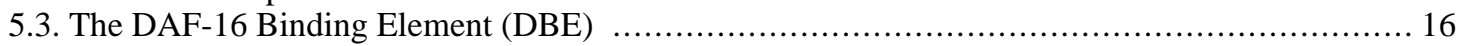

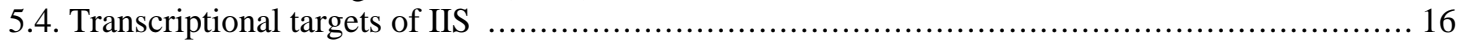

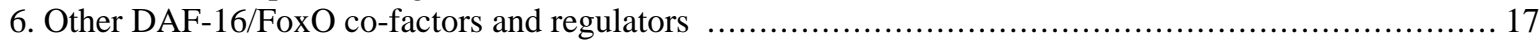

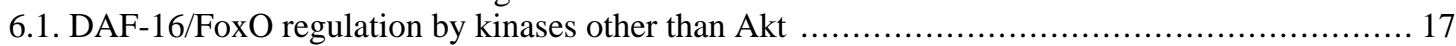

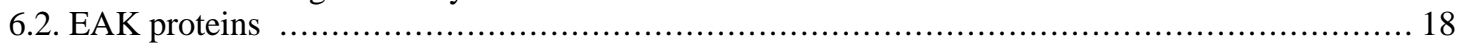

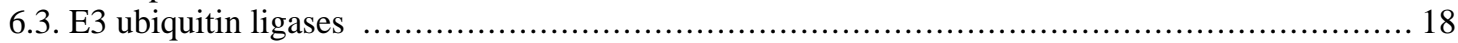

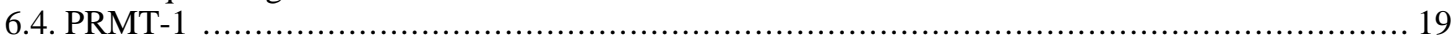

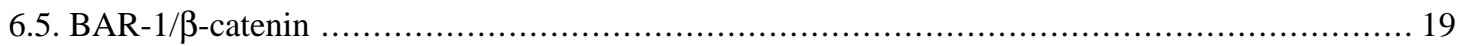

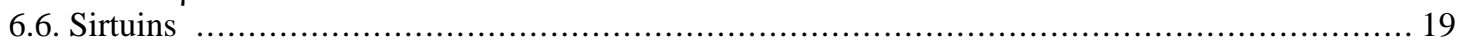

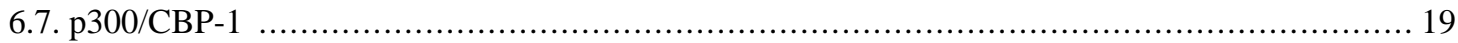

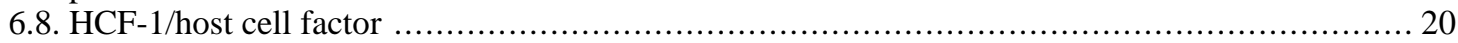

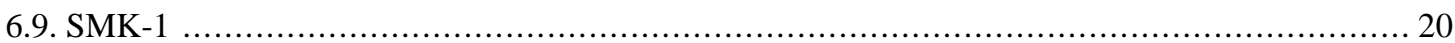

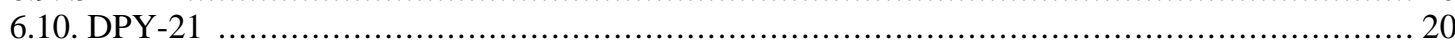

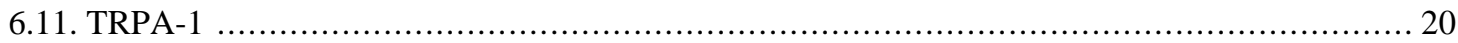

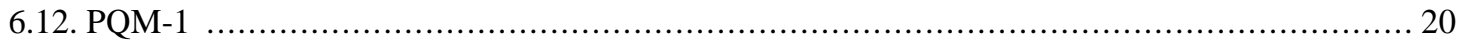

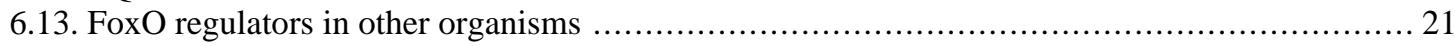

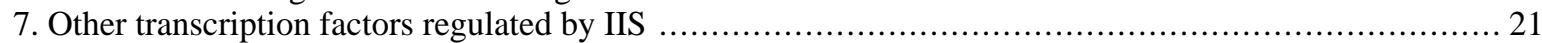

8. Components of IIS in other organisms that are conserved in C. elegans .................................. 22

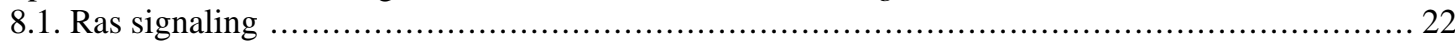

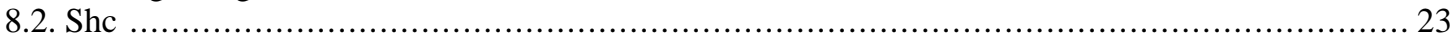

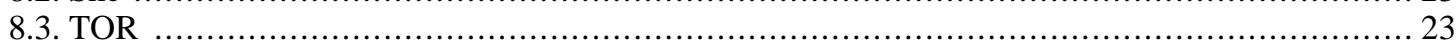

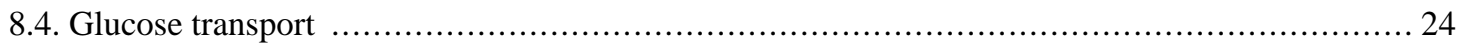

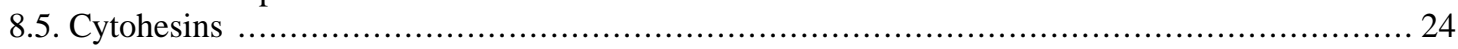

9. System-wide integration of IIS: non-autonomy and interaction with other pathways ...................... 24

9.1. IIS-mediated cell non-autonomous regulation of longevity, dauer formation, metabolism, and

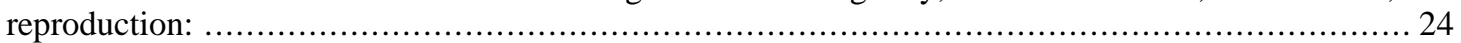

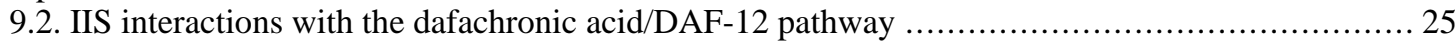

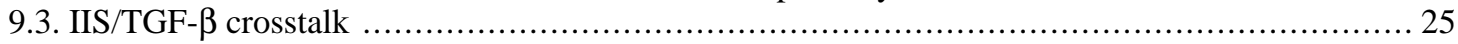

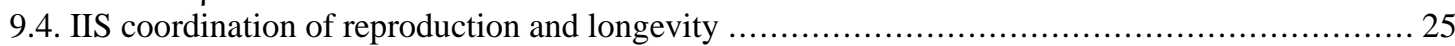

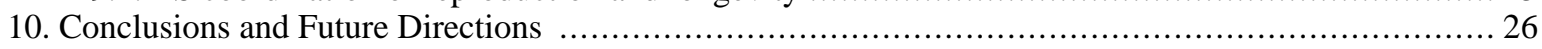

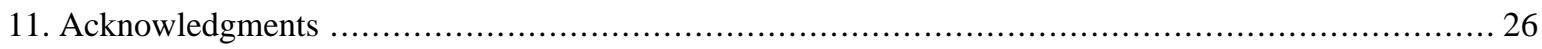

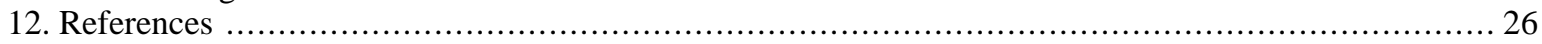

\begin{abstract}
The $C$. elegans insulin/IGF-1 signaling (IIS) pathway connects nutrient levels to metabolism, growth, development, longevity, and behavior. This fundamental pathway is regulated by insulin-like peptide ligands that bind to the insulin/IGF-1 transmembrane receptor (IGFR) ortholog DAF-2. DAF-2/IGFR controls the activity of a conserved phosphoinositide 3-kinase (PI3K)/Akt kinase cascade, culminating in the regulation of a FoxO transcription factor, DAF-16, that governs most of the functions of this pathway. In light of the evolutionary conservation of the IIS pathway, its study in C. elegans is likely to shed light on its functions and regulation in higher organisms, including humans. Originally identified based on its role in the regulation of larval development and aging, IIS also controls a host of other biological processes. Here we review what is currently known about the biological functions and the molecular components of $C$. elegans IIS.
\end{abstract}

\title{
1. Introduction
}

Insulin and insulin-like growth factor-1 (IGF-1) exert their biological effects by binding to and activating cell surface transmembrane receptors with intrinsic tyrosine kinase activity (Ullrich et al., 1985; Ullrich et al., 1986). Activated insulin and IGF-1 receptors phosphorylate a variety of substrates, among which are the IRS (Insulin Receptor Substrate) family of scaffold proteins (White, 1998). Tyrosine-phosphorylated IRS proteins promote the recruitment and activation of components of downstream cascades such as the phosphoinositide 3-kinase 
(PI3K)/Akt, Ras/MAPK (mitogen-activated protein kinase), and mTOR pathways (Taniguchi et al., 2006). These and other insulin and IGF-1 signaling (IIS) pathway components are widely conserved in metazoans (Pinero Gonzalez et al., 2009). Moreover, the role of the IIS in connecting system-wide functions, such as growth, reproduction, and aging, to nutritional status is both conserved and universally important. The use of the genetically tractable system, C. elegans, to study the function of IIS has been valuable, yielding many important insights into the functions, regulation, and outputs of IIS.

Here we review the discovery of the C. elegans IIS pathway and its components (Section 2), phenotypes of IIS mutants (Section 3), molecular characterization of IIS pathway components (Section 4), the FoxO transcription factor DAF-16 (Section 5), DAF-16/FoxO co-factors and regulators (Section 6), other transcription factors regulated by IIS (Section 7), components of IIS in other organisms that are conserved in C. elegans (Section 8), and non-autonomous regulation and integration of insulin signaling (Section 9). We conclude by exploring some prominent remaining questions in the field (Section 10).

\section{The IIS pathway}

\subsection{Components of the IIS pathway}

The main components of the $C$. elegans IIS pathway (Figure 1) include insulin-like peptides (ILPs), at least one of which can bind to and activate the human insulin receptor (Hua et al., 2003). DAF-2/IGFR activation results in recruitment and activation of the phosphoinositide 3-kinase AGE-1/PI3K. In turn, the serine/threonine kinases PDK-1, AKT-1, and AKT-2 are activated, resulting in phosphorylation of the DAF-16/FoxO transcription factor. Phosphorylation of DAF-16/FoxO regulates its interactions with the 14-3-3 proteins PAR-5 and FTT-2, which control DAF-16/FoxO subcellular localization. The DAF-18/PTEN lipid phosphatase and the serine/threonine phosphatase PPTR-1/PP2A counteract AGE-1/PI3K and AKT-1 signaling, respectively. These components will be described in detail below (Section 4). DAF-16/FoxO interacts with additional factors in the nucleus, including SIR-2.1 and HCF-1 (Section 6), as well as additional transcription factors, such as HSF-1 and SKN-1 (Section 7).

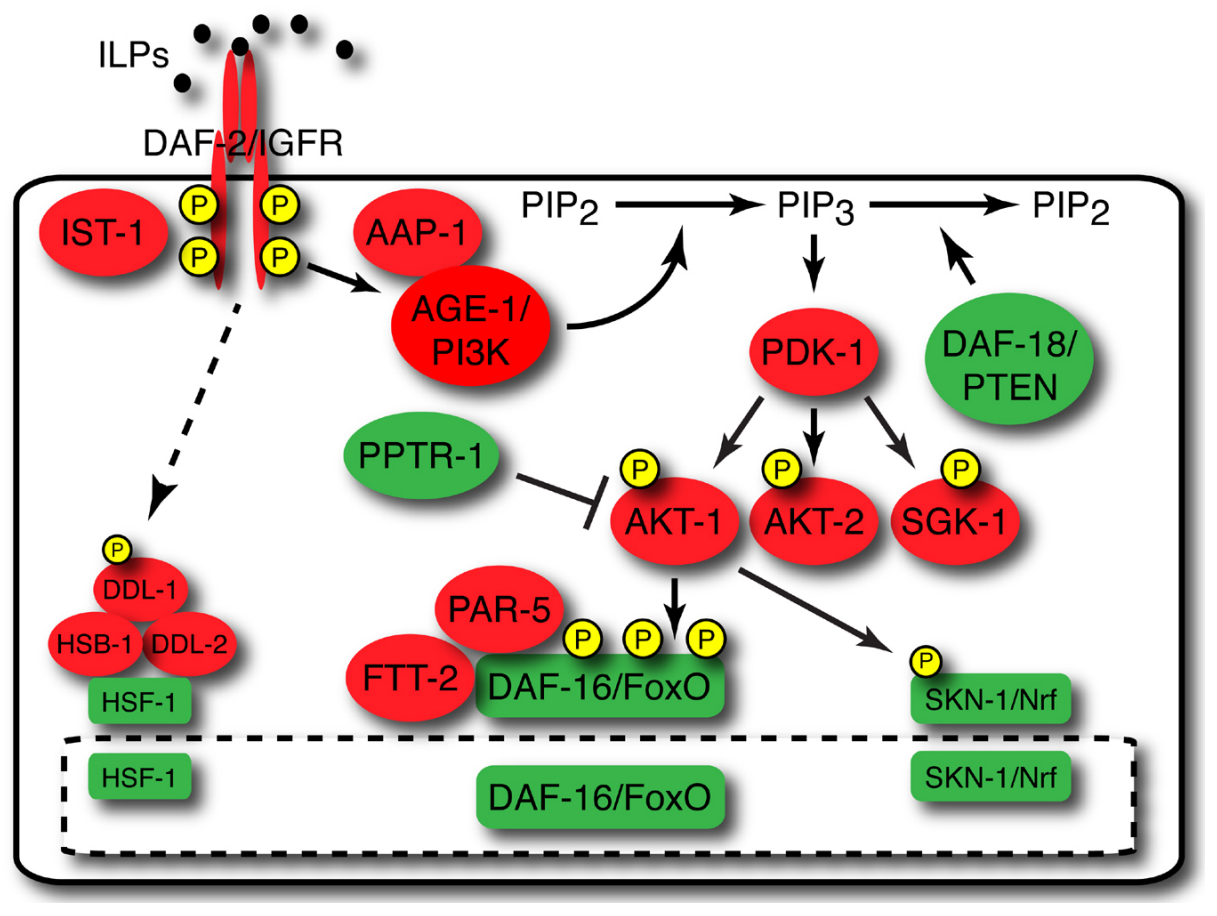

Figure 1. Schematic of $\boldsymbol{C}$. elegans IIS. Active IIS promotes the phosphorylation-dependent cytoplasmic sequestration of the transcription factors DAF-16/FoxO, HSF-1, and SKN-1/Nrf. The insulin/IGF-1 receptor ortholog DAF-2 and other pathway components that promote IIS are colored red, and molecules that either antagonize IIS or are antagonized by IIS are colored green. Insulin-like peptides, which may either promote or antagonize DAF-2 activity, are colored black. See text for details. Abbreviations: ILPs, insulin-like peptides; PI3K, phosphoinositide 3-kinase; PIP, phosphatidylinositol 4,5-bisphosphate; $\mathrm{PIP}_{3}$, phosphatidylinositol 3,4,5-trisphosphate. 


\subsection{Discovery of the $C$. elegans IIS pathway}

The discovery of the IIS pathway in $C$. elegans emerged from converging lines of investigation in several laboratories studying dauer regulation and the genetic control of longevity.

In genetic screens pioneered by the Riddle lab and subsequently performed in the Thomas and Ruvkun labs, dauer-constitutive (Daf-c) and dauer-defective (Daf-d) mutants were isolated (Riddle et al., 1981; Dauer). Among these were mutants that defined the Daf-c genes daf-2 and daf-23 and the Daf-d gene daf-16. The dauer-constitutive phenotype caused by mutations in $d a f-2$ and $d a f-23$ required the activity of the dauer-defective genes daf- 16 and daf-18 (Vowels and Thomas, 1992; Gottlieb and Ruvkun, 1994; Ogg and Ruvkun, 1998; Gil et al., 1999; Mihaylova et al., 1999; Rouault et al., 1999), indicating that DAF-2 and DAF-23 act to prevent dauer arrest in non-dauer-inducing conditions by antagonizing DAF-16 and DAF-18. Genetic analysis indicated that DAF-2 and DAF-23 likely acted in a single pathway to inhibit DAF-16 activity.

In parallel, the labs of Klass and Johnson isolated and characterized the first long-lived C. elegans mutant, age-1. age-1 was first identified by Klass in a genetic screen for long-lived animals (Klass, 1983), and subsequent analysis by the Johnson lab suggested that the longevity phenotype in this mutant strain was due to a recessive mutation in a single gene (Friedman and Johnson, 1988b; Friedman and Johnson, 1988a; Johnson, 1990). Later, Kenyon, et al. found that daf-2 mutants also live twice as long as wild-type animals, and that daf-16 is required for the extended longevity of daf-2 mutants (Kenyon et al., 1993). Molecular analysis demonstrated that age-1 and daf-23 are allelic and that $d a f-2$, age-1/daf-23, and daf-16 encode components of a conserved insulin-like signaling pathway (Morris et al., 1996; Kimura et al., 1997; Lin et al., 1997; Ogg et al., 1997). The extension of life span by mutations in age-1 also required wild-type daf-16 function (Murakami and Johnson, 1996), and the discovery that the life span extension caused by daf-23 mutations also requires daf-16 and daf-18 suggested that the DAF-2/AGE-1/DAF-23/DAF-16 pathway functioned at a crossroads between dauer regulation and longevity (Dorman et al., 1995; Larsen et al., 1995). Intriguingly, despite the natural link between dauer longevity (Klass and Hirsh, 1976) and adult longevity, the long lifespan of daf-2 mutants does not require passing through the dauer stage (Kenyon et al., 1993). Taken together, these results defined a genetic pathway in which DAF-2 and DAF-23/AGE-1 control dauer arrest and life span by acting in opposition to DAF-16 and DAF-18.

\subsection{Conservation of IIS components}

Prior to the availability of genome sequences for multiple organisms, the discovery that the longevity and dauer formation mutants $d a f-2$ and age-1/daf-23 encode C. elegans orthologs of mammalian insulin/IGF-1 receptors (IGFRs) and PI 3-kinase (PI3K), respectively (Morris et al., 1996; Kimura et al., 1997), established the evolutionary conservation of IIS in metazoans and provided seminal insights into the role of IIS in controlling dauer arrest and adult life span. Furthermore, this finding suggested that mobilizing the power of C. elegans as a experimental system to study IIS could lead to new discoveries that would inform investigations into mammalian IIS - a prediction borne out shortly thereafter by the revelation that the $C$. elegans FoxO transcription factor DAF-16 is a major target of IIS (Lin et al., 1997; Ogg et al., 1997). Subsequent studies have revealed new regulatory components and mechanisms involved in IIS regulation of a variety of biological processes. Moreover, many of the downstream transcriptional targets that control these processes have been identified.

\section{IIS pathway loss-of-function phenotypes}

IIS mutants exhibit a wide array of phenotypes, including developmental, reproductive, life history, and behavioral changes, with varying penetrance and expressivity. While the vast majority of early studies on IIS in $C$. elegans focused on its roles in the control of dauer arrest and aging, IIS has since been implicated in a wide variety of biological functions. daf-2/IGFR mutants, in addition to their dauer-constitutive and increased adult longevity (Age) phenotypes, are more resistant to stress and exhibit increased intrinsic thermotolerance (Itt) (Lithgow et al., 1994). Some alleles exhibit additional defects in development, behavior, fat storage, and reproduction (Gems et al., 1998), as described in greater detail below. 


\subsection{IIS regulation of development: dauer, L1 arrest, and germline proliferation}

\subsubsection{IIS regulation of dauer arrest}

Components of $C$. elegans IIS first emerged from forward genetic screens designed to identify mutants with abnormal regulation of dauer arrest (Riddle et al., 1981). A detailed description of the role of IIS in dauer regulation is available in the Dauer chapter of WormBook.

Reduced IIS results in a dauer-constitutive phenotype. Whereas wild-type animals cultured in replete conditions develop into reproductive adults at $25^{\circ} \mathrm{C}$ and exhibit low levels of dauer arrest at $27^{\circ} \mathrm{C}$, mutants with reduced IIS show enhancement of dauer arrest at both temperatures when exposed to the same conditions. Strong daf-2/IGFR loss-of-function, age-1/PI3K null, and akt-1; akt-2 double null mutants all arrest non-conditionally as dauers (Morris et al., 1996; Kimura et al., 1997; Gems et al., 1998; Oh et al., 2005; Alam et al., 2010), and these dauer-constitutive phenotypes are suppressed by daf-16/FoxO null mutations (Vowels and Thomas, 1992; Gottlieb and Ruvkun, 1994; Larsen et al., 1995). Therefore, the conserved PI3K/Akt pathway is the major DAF-2/IGFR output that regulates dauer arrest, and it does so by inhibiting DAF-16/FoxO.

In dauers, the vulval precursor cells (VPCs) that generate the hermaphrodite vulva are reprogrammed to pluripotency, allowing normal vulval development to proceed upon dauer exit. DAF-16/FoxO is specifically required in dauers for VPC reprogramming, acting cell autonomously to prevent VPC specification and maintain pluripotency by blocking inductive (EGFR) and lateral (LIN-12/Notch) signaling (Karp and Greenwald, 2013).

\subsubsection{IIS regulation of $L 1$ arrest}

Besides its critical function in dauer regulation, DAF-2/IGFR activity also controls development and starvation-induced non-dauer larval arrest, largely in a daf-16/FoxO-dependent fashion. daf-2/IGFR mutants develop at a slower rate than wild-type animals (Ruaud et al., 2011), and overexpression of DAF-16/FoxO slows developmental rates (J. Ashraf and C. T. Murphy, unpublished), suggesting that the larval developmental process is regulated by DAF-16/FoxO and its targets even under normal conditions.

IIS also plays a role in regulating the developmental response to limiting conditions in non-dauer larvae. When $C$. elegans eggs hatch, first-stage (L1) larvae undergo developmental arrest if food is absent. This phenomenon is known as "L1 arrest." daf-2/IGFR mutants have a partially penetrant L1 arrest phenotype even in the presence of food. In the absence of food, L1-arrested daf-2/IGFR mutant larvae survive starvation more readily than wild-type L1 larvae. daf-16/FoxO mutation suppresses both the L1 arrest phenotype of daf-2/IGFR mutants in the presence of food as well as the prolonged survival of $d a f-2 / I G F R$ mutant L1 larvae in the absence of food, and DAF-16/FoxO is also required for the survival of L1-arrested wild-type larvae. Therefore, the role of DAF-16/FoxO in promoting survival in starvation-induced L1 arrest is in accord with its role in promoting dauer arrest in response to environmental stressors.

\subsubsection{IIS regulation of cell proliferation in arrested states}

Developmental arrest in L1 or dauer larvae is accompanied by cessation of developmentally programmed cell divisions. These cell divisions are regulated by IIS through at least two mechanisms. In starvation-induced L1 arrest, cells in the $\mathrm{V}$ and $\mathrm{M}$ lineages that stop dividing in wild-type larvae continue to divide in daf-16/FoxO mutant larvae, indicating that DAF-16/FoxO promotes cell cycle arrest (Baugh and Sternberg, 2006). DAF-16/FoxO may inhibit cell division by upregulating the $\mathrm{p} 27^{\mathrm{kip} 1}$ cyclin-dependent kinase inhibitor $c k i-1$, as induction of $c k i-1$ transcription in response to starvation is abrogated by daf-16/FoxO mutation (Baugh and Sternberg, 2006). DAF-16/FoxO may also contribute to the starvation-induced transcriptional repression of lin-4, a microRNA induced during the L1 larval stage that orchestrates the temporal program of larval cell divisions in replete conditions by repressing LIN-14 and LIN-28 translation (Wightman et al., 1993; Moss et al., 1997; Baugh and Sternberg, 2006). It is likely that DAF-16/FoxO regulates the expression of other genes that in turn regulate cell division, development, and growth (Baugh and Sternberg, 2006).

By contrast, cell cycle arrest of the $\mathrm{Z} 2$ and $\mathrm{Z} 3$ primordial germ cells in starved L1 larvae is controlled by a distinct mechanism. Whereas most somatic cells that arrest in starved L1 larvae do so prior to replicating their DNA, Z2 and Z3 arrest in the G2 phase of the cell cycle (Fukuyama et al., 2006). Mutations that inactivate DAF-18/PTEN cause continued proliferation of Z2 and Z3 in arrested L1 larvae, and arrest was restored in age-1; daf-18 and daf-18; akt-1 double mutants. Therefore, PI3K/Akt signaling controls starvation-induced Z2/Z3 arrest. Surprisingly, 
DAF-16/FoxO was not required for Z2/Z3 arrest under these conditions, indicating that AGE-1/PI3K and AKT-1 control Z2/Z3 proliferation independently of DAF-16/FoxO (Fukuyama et al., 2006). Whether DAF-2/IGFR is the activating input to AGE-1/PI3K in this context is not known.

Analogously, DAF-18/PTEN also controls germline proliferation in dauers. Germline arrest in daf-2/AGFR mutant dauers requires DAF-18/PTEN, as daf-2(e1370); daf-18(e1375) double mutant dauers have three times as many germ cells as daf-2(e1370) dauers (Narbonne and Roy, 2006). This is likely due at least in part to increased activation of the PI3K/Akt pathway, as an akt-1 gain-of-function mutation also increased germline proliferation in daf-2(e1370) dauers. The role of DAF-16/FoxO in this context is difficult to assess, as daf-16/FoxO mutation suppresses daf-2(e1370) dauer arrest. However, analysis of daf-7(e1372) dauers, which are dauer-constitutive due to reduced TGF- $\beta$-like signaling (Ren et al., 1996; Schackwitz et al., 1996), revealed that, whereas daf-7; daf-18 double mutant dauers exhibit increased germline proliferation, daf-16/FoxO mutation does not influence germline arrest in daf-7 mutant dauers (Narbonne and Roy, 2006). Taken together, these findings suggest DAF-16/FoxO-independent outputs of PI3K/Akt signaling may play a general role in regulating germline proliferation in arrested larvae.

\subsubsection{IIS regulation of germline proliferation in adulthood}

In the adult, the only mitotically proliferating cells are in the germline, and IIS plays a critical role in regulating the development and maintenance of the proliferative process. Excessive proliferation can result in germline "tumors" in mutants in which meiotic germ cells re-enter the mitotic cell cycle and overproliferate (Francis et al., 1995). Such germline "tumors" are reduced in daf-2/IGFR mutants in a daf-16/FoxO-dependent manner, and downstream targets of DAF-16/FoxO influence germline cell proliferation and p53-dependent apoptosis (Pinkston-Gosse and Kenyon, 2007). This effect is likely due to the role of IIS in promoting Notch-independent germline cell proliferation and cell cycle activity during normal development in reproductive larval stages (Michaelson et al., 2010).

daf-2/IGFR-dependent mitotic proliferation in the germline is regulated by the ILPs INS-3 and INS-33, which are expressed primarily in the nervous system and hypodermis, respectively. These ILPs do not affect dauer formation or intestinal DAF-16/FoxO activity (Michaelson et al., 2010), suggesting that the activity of INS-3 and INS-33 is restricted to daf-2/IGFR-mediated regulation of germline proliferation. Notably, INS-33 is also a direct target of LIN-14 (Hristova et al., 2005), the L1 transcriptional repressor that is itself a target of the lin-4 microRNA (Arasu et al., 1991; Lee et al., 1993; Olsen and Ambros, 1999), perhaps suggesting the presence of a regulatory loop.

daf-2/IGFR mutants also maintain their germline cell proliferation and morphology better with age than wild-type animals (Garigan et al., 2002; Luo et al., 2010; Luo and Murphy, 2011).

\subsection{IIS regulation of longevity and aging}

The isolation and characterization of age-1(hx546) as a long-lived mutant (Klass, 1983; Friedman and Johnson, 1988b; Friedman and Johnson, 1988a; Johnson, 1990) and the subsequent demonstration that daf-2/IGFR mutants live twice as long as wild-type animals (Kenyon et al., 1993) established the importance of IIS in life span control. The $d a f-2 / I G F R$ mutant longevity phenotype does not require passing through the dauer state, as animals are long-lived when shifted to the restrictive temperature at the L4 larval stage, after both the dauer stage (L3 equivalent) and the dauer decision point late in the L1 larval stage (Kenyon et al., 1993). Cynthia Kenyon's lab also showed that RNAi inactivation of daf-2/IGFR in early to mid-adulthood could extend life span, while treatment in the L3 larval stage affected progeny number, clearly delineating the temporal regulation of dauer formation, reproduction, and longevity by DAF-2/IGFR (Dillin et al., 2002).

The AGE-1 PI3K downstream of DAF-2/IGFR also regulates longevity. Homozygous age-1/PI3K mutant progeny of heterozygous mothers live longer than wild-type animals (Morris et al., 1996). Furthermore, when non-maternally rescued homozygotes harboring the strong age-1/PI3K alleles $m g 44$ and $m 333$ (Larsen et al., 1995; Morris et al., 1996) are grown at low temperatures, they exhibit severe developmental delays and extreme longevity, living up to ten times longer than wild-type animals (Ayyadevara et al., 2008). As is the case for daf-2/IGFR mutants, the longevity phenotypes of age-1/PI3K mutants are largely dependent on daf-16/FoxO (Kenyon et al., 1993; Larsen et al., 1995; Morris et al., 1996; Murakami and Johnson, 1996; Ayyadevara et al., 2008). 
IIS regulation of longevity is not restricted to nematodes; similar effects are seen in flies (Clancy et al., 2001; Tatar et al., 2001) and mice (Bluher et al., 2003; Holzenberger et al., 2003). The role of IIS in life span control may also extend to humans. Studies in cohorts of Ashkenazi Jewish centenarians have identified non-synonymous polymorphisms in the gene encoding the IGF-I receptor that are associated with longevity (Suh et al., 2008). Importantly, cells expressing IGF-1 receptors harboring these polymorphisms exhibit reductions in ligand-dependent Akt phosphorylation, gene regulation, and cell cycle progression compared to cells expressing wild-type IGF-1 receptors, indicating that a reduction in IGF-1 signaling is correlated with longevity in humans (Tazearslan et al., 2011). Thus, important downstream functions of IIS may be conserved. Moreover, longevity and phenotypes associated with healthy aging in humans, including cognitive function and metabolic profiles, have been reported to be associated with non-coding genetic variation in FOXO3A (Willcox et al., 2008). The impact of these non-coding polymorphisms on FoxO3A function is not known.

\subsection{IIS regulation of stress resistance}

In addition to their dauer formation and longevity phenotypes, daf-2/IGFR mutants retain their mobility and healthy appearance longer than wild-type animals (Kenyon et al., 1993; Garigan et al., 2002; Herndon et al., 2002). They are resistant to a diverse array of stresses, including heat (Lithgow et al., 1994; Lithgow et al., 1995; Walker et al., 1998; Babar et al., 1999; Walker et al., 2001; Hsu et al., 2003), oxidative stress (Honda and Honda, 1999, 2002), hypoxia (Scott et al., 2002), osmotic stress (Lamitina and Strange, 2005), heavy metal toxicity (Barsyte et al., 2001), ultraviolet radiation (Murakami and Johnson, 1996), and proteotoxicity (Hsu et al., 2003; Morley and Morimoto, 2004; Cohen et al., 2006; Keowkase et al., 2010; Ching et al., 2011; Teixeira-Castro et al., 2011; Zhang et al., 2012). Most of this stress resistance is mediated by direct DAF-16/FoxO targets (see Section 5.4).

daf-2/IGFR mutants are also more immune to pathogenic bacterial infections than are wild-type animals (Garsin et al., 2003; Kerry et al., 2006), again due to the regulation of DAF-16/FoxO target genes (Section 5.4). Intriguingly, daf-2/IGFR mutants also exhibit enhanced RNAi (Wang and Ruvkun, 2004); as the C. elegans RNAi machinery is required for antiviral defense (Felix et al., 2011), daf-2/IGFR mutants might also be more resistant to viral infection (although this has not yet been shown).

\subsection{IIS regulation of fat metabolism}

daf-2/IGFR mutants have increased fat content compared to wild-type animals (Kimura et al., 1997; Ogg et al., 1997; Ashrafi et al., 2003; Perez and Van Gilst, 2008; O'Rourke et al., 2009). Imaging of live animals using coherent anti-Stokes Raman scattering (CARS) indicates that this increase is due at least in part to greater fat accumulation in the hypodermis (Hellerer et al., 2007). In vivo labeling of fat stores with ${ }^{13} \mathrm{C}$-enriched $E$. coli revealed that increased de novo lipogenesis largely accounts for the increased fat phenotype of daf-2/IGFR mutants (Perez and Van Gilst, 2008). The increased fat storage phenotype of daf-2/IGFR mutant animals is dependent on daf-16/FoxO (Ogg et al., 1997; Ashrafi et al., 2003; Perez and Van Gilst, 2008). Several downstream targets of DAF-16/FoxO likely contribute to changes in fat metabolism, including fat- genes that act in fatty acid desaturation and lipolysis, acyl-CoA and alcohol dehydrogenases, glyoxylate cycle regulators, and autophagy regulators (Melendez et al., 2003; Murphy et al., 2003; Lapierre et al., 2011; Lapierre et al., 2012) (see Section 5.4 for details). An analysis of several independent long-lived daf-2/IGFR mutants suggests that alterations in lipogenesis may be somewhat uncoupled from longevity (Perez and Van Gilst, 2008), suggesting that factors other than fat levels may ultimately determine life span.

\subsection{IIS regulation of reproductive aging}

The extended germline maintenance of $d a f-2 / I G F R$ mutants, coupled with their extension of oocyte quality maintenance (Luo et al., 2010), results in the greatly extended reproductive span of daf-2/IGFR mutants (Hughes et al., 2007; Luo et al., 2009; Luo et al., 2010), again highlighting the role of IIS in regulating mitotic cell maintenance as well as post-mitotic survival and stress responses. The cell autonomy of IIS activity in reproductive maintenance differs from that of germline proliferation during development: while DAF-2/IGFR-mediated proliferation requires DAF-16/FoxO activity in the germline (Michaelson et al., 2010), maintenance of reproduction with age requires DAF-16/FoxO activity in muscle and intestine (Luo et al., 2010), the same tissue that provides the bulk of the longevity-promoting activity of DAF-16/FoxO (Libina et al., 2003). The contribution of muscle DAF-16/FoxO activity to reproductive longevity was a surprise, as this tissue plays no role in somatic longevity (Libina et al., 2003). Interestingly, Michaelson et al. also report a minor contribution from DAF-16/FoxO activity in muscle in regulation of germline proliferation during development (Michaelson et al., 2010). 


\subsection{IIS and germline/gonad regulation of life span}

The state of germline proliferation is, in turn, communicated to the soma to influence organismal longevity. Hsin and Kenyon discovered that ablation of germline precursor cells increases life span, while ablation of the surrounding somatic gonad precursors, which also causes sterility, suppresses this longevity phenotype (Hsin and Kenyon, 1999). These results suggest that two opposing longevity signals are communicated from the germline and somatic gonad (Hsin and Kenyon, 1999; Yamawaki et al., 2008; Yamawaki et al., 2010). The long life of germline-ablated animals requires daf-16/FoxO (Hsin and Kenyon, 1999) and pha-4/FoxA (Lapierre et al., 2011), as well as daf-12 (Hsin and Kenyon, 1999) and $n h r-80$ (Goudeau et al., 2011), which encode conserved nuclear receptors. Germline ablation activates intestinal DAF-16/FoxO by promoting its nuclear translocation, while the somatic gonad signal is dependent on daf-2/IGFR (Hsin and Kenyon, 1999; Berman and Kenyon, 2006). Further communication with DAF-16/FoxO in somatic tissues is mediated by such factors as KRI-1, an ankyrin and FERM-domain protein (Berman and Kenyon, 2006), and TCER-1, a transcription elongation factor (Ghazi et al., 2009), both of which are required for germline-mediated longevity. Nuclear localization and activation of DAF-16/FoxO in animals lacking a germline requires the nuclear receptor DAF-12 as well as dafachronic acids (DAs), which are steroid hormone ligands for DAF-12 (Berman and Kenyon, 2006; Gerisch et al., 2007) (see Section 9.2).

Intriguingly, although both IIS and the germline control life span by regulating DAF-16/FoxO activity, reducing IIS in animals lacking a germline extends life span substantially (Arantes-Oliveira et al., 2003). Thus, the mechanisms by which IIS and the germline regulate DAF-16/FoxO activity might be distinct to some degree. This is underscored by the observation that KRI-1 and TCER-1 are required for life span extension caused by germline ablation, but are dispensable for longevity in the context of reduced IIS (Berman and Kenyon, 2006; Ghazi et al., 2009). Germline proliferation longevity signals regulate the expression of genes both dependent on and independent or upstream of DAF-16/FoxO activity (McCormick et al., 2011). The dafachronic acid pathway makes up one arm of this regulation: in animals lacking a germline, daf-36 expression is induced, generating DA, in turn activating DAF-12. The let-7 family miRNAs mir-84 and mir-241 are induced by DAF-12, and in turn down regulate AKT-1 and LIN-14, resulting in the activation of DAF-16/FoxO (Shen et al., 2012). The miRNA mir-71, which is upregulated with age and promotes longevity (de Lencastre et al., 2010), is required for the long lifespan of animals lacking a germline; mir-71 acts in the nervous system to promote longevity, upstream of DAF-16/FoxO activity and independently of daf-12 (Boulias and Horvitz, 2012). The mir-71 targets that are important for life span control are not known.

Germline stem cell loss in Drosophila also increases life span through IIS (Flatt et al., 2008), suggesting that the germline/gonad communication via IIS to regulate somatic longevity is evolutionarily conserved. Such communication from the soma to the germline, and from the germline to the soma (Hsin and Kenyon, 1999; Luo et al., 2010), might enable an organism to tune its reproductive span and life span properly to adapt to changing nutrient availability (Luo and Murphy, 2011). This system-wide signaling integration is discussed in more detail in Section 9.

\subsection{IIS and neuronal/behavioral phenotypes}

IIS mutants also exhibit reduced rates of neural decline compared to wild-type animals, as indicated by a number of behavioral phenotypes (see Stein and Murphy 2012 for greater detail). They exhibit extended isothermal tracking (Murakami et al., 2005), motor activity (Duhon and Johnson, 1995; Huang et al., 2004; Hsu et al., 2009), and positive butanone associative learning with age (Kauffman et al., 2010). Touch receptor neurons and VNC cholinergic axons of $d a f-2 / I G F R$ mutants also show delayed progression of age-dependent morphological defects, while such defects are accelerated in daf-16/FoxO mutants (Pan et al., 2011). daf-2/IGFR mutant animals also exhibit delayed ectopic neurite branching compared to age-matched wild-type animals, again in a daf-16/FoxO-dependent manner (Tank et al., 2011). Additionally, daf-2/IGFR mutants exhibit improved short- and long-term associative memory early in adulthood compared to wild-type animals (Kauffman et al., 2010). While the latter function is due to elevated CREB levels in daf-2/IGFR mutants (Kauffman et al., 2010), the molecular basis for increased short-term associative memory and extended learning ability in daf-2/IGFR mutant animals is not yet known.

By contrast, other learning behaviors are impaired in IIS mutants. Specifically, salt chemotaxis learning appears to require functional IIS, as calcium signaling in the ASER neuron, which senses salt, is altered in IIS mutants (Tomioka et al., 2006; Vellai et al., 2006; Lin et al., 2010; Oda et al., 2011). daf-2/IGFR mutant chemotaxis 
to salt prior to training (naïve) is higher than that of wild-type as well (Tomioka et al., 2006; Oda et al., 2011). Cell-specific rescue experiments suggest that DAF-2/IGFR and AGE-1/PI3K act in the ASER, while the ILP INS-1 acts in the AIA interneurons, which have synaptic outputs to ASER (Tomioka et al., 2006; Oda et al., 2011). IIS mutants are also defective in a paradigm that pairs the positive chemoattractant benzaldehyde with starvation to create a negative association (Lin et al., 2010). ins-1 and daf-2/IGFR mutants, which display normal positive "butanone enhancement" learning (Torayama et al., 2007; Kauffman et al., 2010), are defective in benzaldehyde-starvation avoidance learning (Lin et al., 2010), an effect that is dependent upon daf-18/PTEN but only partially dependent on $d a f-16 / F o x O$. It is possible that insulin signals are required in these negative associative paradigms to properly signal the starved state; if the responses of IIS mutants to starvation and salt stress are muted, then their ability to form associations with these negative states might be hindered (Stein and Murphy, 2012).

\section{Molecular characterization of IIS pathway components}

\subsection{DAF-2, the $C$. elegans insulin/IGF-1 receptor ortholog}

Cloning of $d a f-2$ revealed that it encodes a receptor tyrosine kinase that shares greater than $30 \%$ amino acid identity with the human insulin, IGF-1, and insulin receptor-related receptors (Kimura et al., 1997). Mammalian insulin receptors form $\alpha 2 / \beta 2$ heterotetramers that contain extracellular ligand-binding domains in the? $\alpha$-subunit and transmembrane and intracellular tyrosine kinase domains in the $\beta$-subunit. Cysteines in both subunits form disulfide bonds that link each $\alpha$-subunit to the extracellular domain of a $\beta$-subunit as well as to the other $\alpha$-subunit of the heterotetramer (Lawrence et al., 2007). Insulin binding causes a conformational change that induces autophosphorylation, activating intrinsic receptor tyrosine kinase activity (Luo et al., 1999; Kido et al., 2001).

daf-2 encodes the sole $C$. elegans insulin receptor family member (Kimura et al., 1997). The daf-2 locus encodes two alternatively spliced transcripts; nothing is known about the specific functions of these DAF-2/IGFR isoforms. DAF-2/IGFR is highly expressed in the nervous system and the XXX neuroendocrine cells, with weaker expression in hypodermis, intestine, and other tissues (Hunt-Newbury et al., 2007; Kimura et al., 2011), and its abundance is modulated by nutritional status (Kimura et al., 2011). Although a family of putative receptors with weak homology to the DAF-2/IGFR extracellular domain has been identified bioinformatically (Dlakic, 2002), their biological significance is not yet known.

\subsection{Insulin-like peptides (ILPs)}

Although the C. elegans genome encodes a single insulin/IGF-1-like receptor, it contains a plethora of genes predicted to encode insulin-like peptides (ILPs). Forty members of this gene family have been identified through genetic and bioinformatic studies (Duret et al., 1998; Kawano et al., 2000; Pierce et al., 2001; Li et al., 2003). Several ILPs have been shown to regulate dauer formation, longevity, and development. A few, such as daf-28 (Li et al., 2003), ins-1 (Pierce et al., 2001), ins-6 (Cornils et al., 2011; Chen et al., 2013b), and ins-7 (Murphy et al., 2007; Chen et al., 2013b), have been studied in some depth, but the biological functions of the vast majority of the ILPs remain unknown at this time. Most ILPs are expressed in neurons (Pierce et al., 2001) or subsets of neurons (Li et al., 2003; Murphy et al., 2007; Cornils et al., 2011; Chen et al., 2013b), but regulation of ILPs outside of neurons has also been observed (Pierce et al., 2001; Murphy et al., 2007), particularly in the intestine (Murphy et al., 2007) and hypodermis (Michaelson et al., 2010). Overexpression of INS-7 in the intestine shortens lifespan and suppresses expression of the DAF-16/FoxO reporter sod-3p::GFP expression in the head, suggesting that it decreases DAF-16/FoxO activity in other tissues (Murphy et al., 2007).

Although most ILPs lack many of the sequence characteristics of human insulin (Pierce et al., 2001), they may still function as receptor ligands. For example, the divergent ILP INS-6, which is predicted to lack a C-peptide, can bind to and activate the human insulin receptor (Hua et al., 2003). Another surprising feature of C. elegans ILPs is their apparent ability to act either as antagonists (INS-1, INS-18, INS-7) or agonists (INS-7) of DAF-2/IGFR (Pierce et al., 2001; Hristova et al., 2005; Murphy et al., 2007; Cornils et al., 2011). The mechanistic basis for these differences in function is not yet understood. One hypothetical model is that alternate DAF-2/IGFR-like co-receptors (Dlakic, 2002) might integrate responses to ILPs in a combinatorial fashion, but such a role for these putative co-receptors has not been uncovered. Additionally, whether ILPs that antagonize DAF-2/IGFR signaling do so through direct as opposed to indirect effects on DAF-2/IGFR activity (e.g., by influencing the processing and maturation of other ILPs) is not yet known. Genes encoding agonist/antagonist pairs (e.g., ins-7 and ins-18), can also be regulated in opposite manners at the expression level (Murphy et al., 2003; Liu et al., 2004; Murphy et al., 2007; Shaw et al., 2007), perhaps to coordinate the downstream IIS response between tissues and pathways (Murphy et al., 2007; Shaw et al., 2007). For example, the feed-forward mechanism of ins-7 repression and ins- 18 induction in the 
intestine activates DAF-16/FoxO in other tissues, thus aligning IIS activity in all tissues to coordinate somatic aging, called "FoxO-to-FoxO" signaling (Murphy et al., 2007). The ins-7/ins-18 insulin pair is also regulated by the DAF-7/TGF- $\beta$ dauer regulatory pathway, linking the two pathways (Liu et al., 2004; Shaw et al., 2007; Narasimhan et al., 2011); this is discussed in greater detail in Section 9.3. In another form of communication called "ILP-to-ILP" signaling, INS-6 in the ASI neuron represses INS-7 expression in the URX neuron; INS-7 antagonizes DAF-2/IGFR in the RIA neuron, disrupting aversive olfactory learning (Chen et al., 2013b).

While most experiments involving ILPs suggest that they signal through DAF-2/IGFR, there is at least one report of a daf-2/IGFR-independent insulin effect, which is ins-1 regulation of AWC sensory neuron activity (Chalasani et al., 2010). The receptor that mediates INS-1 action in this context is not yet known. The observation that mammalian G-protein-coupled receptors can act as receptors for the relaxin family of insulin-related peptide hormones (Hsu et al., 2002) suggests that non- daf-2/IGFR receptors could mediate some of the biological functions of ILPs.

\subsection{Regulation of ILP secretion}

Recent studies have identified both positive and negative regulators of ILP secretion. ASNA-1 is a conserved ATPase that positively regulates DAF-2/IGFR signaling by promoting the secretion of the ILP DAF-28 (Section 4.2) and possibly other ILPs (Kao et al., 2007). Human ASNA1 is expressed specifically in the insulin-producing $\beta$-cells of pancreatic islets and enhances insulin secretion in $\beta$-cell-derived cell lines, suggesting that the role of ASNA-1 in regulating ILP secretion is evolutionarily conserved (Kao et al., 2007). DAF-28 secretion also requires intact mitochondrial function, as knockdown of components of the TOMM mitochondrial protein import complex reduces DAF-28 secretion (Billing et al., 2011). Whether mitochondria communicate with ASNA-1 to promote DAF-28 secretion is not known.

An analysis of C. elegans orthologs of Bardet-Biedl syndrome (BBS) disease genes has revealed a role for BBS proteins in attenuating ILP secretion. BBS is an autosomal recessive genetic disorder of pleiotropic phenotype caused by mutations in any of fourteen genes encoding proteins that are required for the normal function of cilia (Zaghloul and Katsanis, 2009). Strikingly, C. elegans orthologs of BBS disease genes are expressed specifically in ciliated neurons (Ansley et al., 2003). In contrast to mutations in many cilia genes, which reduce DAF-28 secretion, bbs mutations enhance the secretion of DAF-28 and other neuropeptides (Lee et al., 2011). RNAi of murine BBS genes in the pancreatic $\beta$-cell-derived MIN6 cell line induced insulin secretion 2-fold, suggesting that the role of BBS proteins in limiting ILP secretion is evolutionarily conserved.

\subsection{AGE-1/phosphoinositide 3-kinase (PI3K)}

age-1 was first identified in a forward genetic screen for long-lived mutants (Klass, 1983; Friedman and Johnson, 1988b; Friedman and Johnson, 1988a; Johnson, 1990). As is the case for daf-2/IGFR mutants, the extended longevity of age-1 mutants requires both daf-16 and daf-18, suggesting that daf-2/IGFR and age-1 act in the same pathway to regulate longevity (Dorman et al., 1995). The age-1(hx546) mutant that emerged from the screen for long-lived mutants also forms dauers at high temperatures $\left(27^{\circ} \mathrm{C}\right)$ (Malone et al., 1996; Morris et al., 1996). Both the extended life span and dauer-constitutive phenotypes of age-l(hx546) were mapped to the same genomic region as the dauer-constitutive phenotype of daf-23 mutants, and age-1(hx546) does not complement daf-23 mutants (Malone et al., 1996; Morris et al., 1996), suggesting that age-1 and daf-23 are allelic. Cloning of age-1 confirmed this notion, as age-1 and daf-23 mutants contain molecular lesions in an open reading frame that encodes the C. elegans ortholog of the p110 catalytic subunit of Class IA phosphoinositide 3-kinases (PI3K) (Morris et al., 1996; Ayyadevara et al., 2008). As null alleles of both daf-2/IGFR and age-1/PI3K undergo non-conditional dauer arrest (Morris et al., 1996; Patel et al., 2008b), AGE-1/PI3K is likely to be the major signaling output of activated DAF-2/IGFR in dauer regulation.

The PI3K superfamily of enzymes phosphorylates phosphoinositides at the 3' position of the inositol ring. Activation of Class I PI3Ks by insulin and IGFs increases 3-phosphoinositide (PIP ${ }_{3}$ ) concentrations at the plasma membrane, thus promoting the recruitment and activation of PIP-dependent signaling complexes (Cantrell, 2001). Mammalian Class IA PI3Ks are heterodimers consisting of a p1310 catalytic subunit and an adaptor subunit that contains two SH2 domains. Upon binding of ligand to insulin and IGF-1 receptors, IRS (insulin receptor substrate) scaffold proteins are phosphorylated on multiple tyrosine residues, creating binding sites for the $\mathrm{SH} 2$ domains of the PI3K adaptor subunit that promote the recruitment of PI3K to the plasma membrane (Taniguchi et al., 2006). 
The $C$. elegans genome encodes a single PI3K adaptor subunit (AAP-1; $\underline{A}$ GE-1 adaptor protein) and a putative IRS homolog (IST-1; insulin receptor substrate homolog) (Wolkow et al., 2002). An aap-1 mutant, aap-1(m889), was isolated in a genetic screen for thermotolerant mutants (Munoz and Riddle, 2003). Similar to age-1/PI3K mutants, aap-1(m889) mutants live long in a daf-16/FoxO-dependent manner (Munoz and Riddle, 2003). Furthermore, AAP-1 protein synthesized in E. coli can bind to an N-terminal fragment of AGE-1/PI3K (Wolkow et al., 2002). However, in contrast to the non-conditional dauer arrest phenotype of age-1/PI3K null mutants, aap-1 $(\mathrm{m} 889)$ animals develop reproductively when cultured at $25^{\circ} \mathrm{C}$ and only undergo dauer arrest at $27^{\circ} \mathrm{C}$ (Munoz and Riddle, 2003). This may be a consequence of the fact that the $m 889$ allele is a nonsense mutation that is predicted to create a truncated AAP-1 protein that still contains the N-terminal SH2 domain as well as the inter-SH2 domain that mediates the association of mammalian p85 with p110 (Munoz and Riddle, 2003). Therefore, the mutant AAP-1 protein synthesized in aap-1 $(\mathrm{m} 889)$ may still be able to interact with AGE-1/PI3K. The aap-1 null phenotype has not been described. Notably, no aap-1 mutant alleles have emerged from genetic screens for dauer-constitutive mutants in which multiple alleles of daf-2/IGFR and age-1/PI3K were isolated. Thus, the possibility exists that DAF-2/IGFR may be able to activate AGE-1/PI3K in the absence of AAP-1.

\subsection{IRS (insulin receptor substrate) proteins}

IST-1 was identified based on comparative sequence analysis with mammalian IRS proteins. IST-1 has limited amino acid similarity to mammalian IRS proteins; the greatest sequence identity lies in the pleckstrin homology and phosphotyrosine binding motifs (Wolkow et al., 2002). Unlike mammalian IRS proteins, which contain several YxxM motifs that can bind to Class IA PI3K adaptor subunits when phosphorylated (Sun et al., 1991; Sun et al., 1995), IST-1 contains a single YxxM motif. ist-1 RNAi does not induce dauer arrest in wild-type animals at any temperature but enhances dauer arrest to a comparable extent in animals harboring either partial loss-of-function or null age-1/PI3K mutants (Wolkow et al., 2002). Although the phenotype of an ist-1 null mutant has not been reported, these results suggest that, in contrast to Drosophila chico (Bohni et al., 1999) and mammalian IRS proteins (Shaw, 2011), IST-1 may function primarily to couple DAF-2/IGFR to signaling outputs that act in parallel to AGE-1/PI3K. ist-1 mutants have also not emerged from dauer-constitutive screens, suggesting that IST-1 may be dispensable for DAF-2/IGFR-dependent activation of AGE-1/PI3K.

\subsection{DAF-18/PTEN}

The PTEN (phosphatase and tensin homolog) phosphatase has been well studied in mammalian systems because of its important role as a tumor suppressor. The dauer-defective mutant daf- 18 was identified as the $C$. elegans PTEN ortholog (Ogg and Ruvkun, 1998; Gil et al., 1999; Mihaylova et al., 1999; Rouault et al., 1999). PTEN is a lipid phosphatase that antagonizes PI3Ks by dephosphorylating PIP at the 3' position of the inositol ring (Hollander et al., 2011). DAF-18/PTEN acts in opposition to AGE-1/PI3K and DAF-2/IGFR, as loss-of-function daf-18/PTEN mutations fully suppress the dauer-constitutive and life span extension phenotypes of age-1/PI3K and daf-2/IGFR loss-of-function mutations (Gottlieb and Ruvkun, 1994; Dorman et al., 1995; Larsen et al., 1995; Ogg and Ruvkun, 1998; Gil et al., 1999; Mihaylova et al., 1999). Although a daf-18/PTEN null mutation suppresses age-1/PI3K and daf-2/IGFR mutant phenotypes to comparable extents (Gil et al., 1999; Mihaylova et al., 1999), the daf-18(e1375) mutation, which is a 30-base-pair insertion in the fourth exon that introduces a premature termination codon downstream of the phosphatase domain (Ogg and Ruvkun, 1998; Gil et al., 1999; Mihaylova et al., 1999; Rouault et al., 1999), fully suppresses age-1/PI3K null phenotypes but does not suppress the dauer-constitutive phenotype of daf-2(e1370) mutants. This is consistent with the differential suppression of age-1/PI3K and daf-2/IGFR mutant phenotypes by akt-1 and $p d k-1$ gain-of-function mutations (see Sections 3.7 and 3.8) (Paradis and Ruvkun, 1998; Paradis et al., 1999) and supports the existence of DAF-2/IGFR outputs that transduce signals in parallel to AGE-1/PI3K.

Most PTEN missense mutations found in human tumors reduce its lipid phosphatase activity (Rodriguez-Escudero et al., 2011); however, PTEN can also dephosphorylate proteins (Downes et al., 2007). Although it is not known whether DAF-18/PTEN has protein phosphatase activity, a DAF-18/PTEN missense mutant corresponding to the mammalian PTEN G129E mutant that lacks lipid phosphatase activity but retains protein phosphatase activity (Furnari et al., 1998; Myers et al., 1998) does not rescue the dauer-defective and life span shortening phenotypes of a daf-18 null mutant, suggesting that DAF-18/PTEN controls dauer arrest and life span by dephosphorylating PIP $_{3}$ (Solari et al., 2005). 


\subsection{AKT-1 and AKT-2}

In mammals, Akt/Protein Kinase B (PKB) family serine/threonine kinases mediate many of the cellular responses to insulin and IGF-1. Ligand-dependent activation of Class I PI3Ks increases PIP levels, resulting in the recruitment of Akt/PKB to the plasma membrane via binding of its $\mathrm{N}$-terminal pleckstrin homology $(\mathrm{PH})$ domain to PIP. Akt/PKB is subsequently activated by phosphorylation at two conserved residues (T308 and S473 in human AKT1) (Taniguchi et al., 2006). Mammalian Akt/PKB directly phosphorylates several substrates (Manning and Cantley, 2007).

The C. elegans genome encodes two Akt/PKB family members, AKT-1 and AKT-2. A gain-of-function allele of akt-1, mg144, emerged from a screen for suppressors of the non-conditional dauer-constitutive phenotype of an age-1/PI3K null mutant (Paradis and Ruvkun, 1998), and loss-of-function akt-1 alleles have been isolated in genetic screens for dauer-constitutive mutants (Ailion and Thomas, 2003). As is the case for age-1/PI3K mutants (Gottlieb and Ruvkun, 1994), the dauer-constitutive phenotype of akt-1 loss-of-function mutants requires daf-16/FoxO activity (Ailion and Thomas, 2003; Hu et al., 2006). Unlike age-1/PI3K and daf-2/IGFR null mutants, which undergo dauer arrest nonconditionally, akt-1 null mutants have a weaker dauer-constitutive phenotype, developing reproductively when cultured at $25^{\circ} \mathrm{C}$ and only undergoing dauer arrest at high penetrance when grown at $27^{\circ} \mathrm{C}$ (Ailion and Thomas, 2003; Hu et al., 2006). Accordingly, akt-1 null mutants also live longer than wild-type animals, though not to the same degree as age-1/PI3K and daf-2/IGFR mutants (Alam et al., 2010). Although akt-2 mutants have not yet emerged from forward genetic screens, akt-2 null mutants do have extended life span compared to wild-type animals (Saul et al., 2009; Alam et al., 2010; Kwon et al., 2010), and inactivation of both akt-1 and akt-2 results in nonconditional dauer arrest and a marked extension of life span that is reminiscent of the age-1/PI3K null phenotype (Oh et al., 2005; Ayyadevara et al., 2008; Alam et al., 2010). Therefore, AKT-1 and AKT-2 are likely the major outputs of AGE-1/PI3K.

Mammalian Akt/PKB isoforms have several substrates, including FoxO family transcription factors (Manning and Cantley, 2007). By contrast, the observation that daf-16/FoxO null mutations fully suppress the dauer-constitutive and life span extension phenotypes of age-1/PI3K null mutants (Gottlieb and Ruvkun, 1994; Dorman et al., 1995; Larsen et al., 1995) indicates that DAF-16/FoxO is the primary target of AGE-1/PI3K, and by extension, AKT-1/2. Akt/PKB family members phosphorylate substrates at serines or threonines within motifs containing arginine residues five and three amino acids $\mathrm{N}$-terminal to the phosphoacceptor residue (RxRxxS/T) (Manning and Cantley, 2007). Akt/PKB inhibits FoxO by inducing FoxO phosphorylation at three sites that lie within conserved RxRxxS/T motifs, thus promoting the sequestration of FoxO in the cytoplasm via binding to 14-3-3 proteins (Brunet et al., 1999). The FoxO RxRxxS/T motifs are conserved in DAF-16, and mutation of these phosphoacceptor sites to alanine in both mammalian FoxO and DAF-16 promotes nuclear translocation in the presence of intact IIS (Brunet et al., 1999; Lin et al., 2001). Accordingly, a functional DAF-16::GFP fusion protein translocates to the nucleus in animals with reduced DAF-2/IGFR or AKT-1 activity (Henderson and Johnson, 2001; Lee et al., 2001; Lin et al., 2001; Zhang et al., 2008; Padmanabhan et al., 2009). Thus, the molecular basis for FoxO transcription factor regulation by Akt/PKB is evolutionarily conserved.

\subsection{PDK-1}

Full activation of Akt/PKB requires phosphorylation of both T308 in the activation segment and S473 in the C-terminal hydrophobic motif (Datta et al., 1999). Both of these sites are conserved in AKT-1 and AKT-2 (T350/S517 in AKT-1A and T337/S505 in AKT-2A, respectively) (Paradis and Ruvkun, 1998), suggesting that the underlying mechanisms governing Akt/PKB activation are conserved. In both mammalian cells and C. elegans, T308 is phosphorylated by the phosphoinositide-dependent kinase Pdk, a serine/threonine kinase that, similar to $\mathrm{Akt} / \mathrm{PKB}$, is recruited to the plasma membrane through binding of its PH domain to PIP (Toker and Newton, 2000). A gain-of-function mutation in the C. elegans Pdk ortholog $p d k-1, m g 142$, was isolated in the same screen in which the akt-1 (mgl44) gain-of-function mutant was isolated (Paradis et al., 1999). pdk-1(mgl42) strongly suppresses the dauer-constitutive phenotype of age-1/PI3K null mutants but does not suppress dauer arrest in animals with reduced akt-1 and akt-2 activity, suggesting that PDK-1 acts downstream of AGE-1/PI3K and upstream of AKT-1 and AKT-2 (Paradis et al., 1999). The suppression of age-1(null) dauer arrest by pdk-1(mgl42) is abrogated by RNAi of either akt- 1 or (to a lesser extent) akt-2, indicating that AKT-1 and AKT-2 are the major targets of PDK-1 in dauer regulation (Paradis et al., 1999). The pdk-1(sa680) loss-of-function mutant has a dauer-constitutive phenotype at $25^{\circ} \mathrm{C}$ and extended longevity; both of these phenotypes require DAF-16/FoxO activity (Paradis et al., 1999). The pdk-1(null) phenotype has not been reported; therefore, it is not known whether AGE-1/PI3K has other outputs in addition to PDK-1 that contribute to the activation of AKT-1 and AKT-2. 
Multiple mammalian kinases, including Rictor/mTORC2 and DNA-dependent protein kinase, have recently been shown to activate Akt/PKB through phosphorylation of its C-terminal hydrophobic motif site (Bozulic and Hemmings, 2009). The C. elegans kinase(s) that phosphorylates this site is not known. To date, no candidates have emerged from genetic screens. Loss-of-function mutations in the C. elegans Rictor ortholog rict-1, identified in screens for mutants with reduced fat content, shorten life span and do not promote dauer arrest under standard conditions (Jones et al., 2009; Soukas et al., 2009), suggesting that, in contrast to mammalian Rictor/mTORC2 (Sarbassov et al., 2005), RICT-1 does not contribute to Akt/PKB activation in dauer regulation or life span control.

Notably, whereas both akt-1 and $p d k-1$ gain-of-function mutations fully suppress the dauer-constitutive phenotype of age-1/PI3K null mutants, they only partially suppress the dauer-constitutive phenotype of daf-2/IGFR mutants (Paradis and Ruvkun, 1998; Paradis et al., 1999). Since DAF-2/IGFR activates AGE-1/PI3K, these results suggest that DAF-2/IGFR activates additional pathways that act in parallel to AGE-1/PI3K to regulate dauer arrest.

\subsection{PPTR-1}

PPTR-1 is the $C$. elegans protein phosphatase 2A (PP2A) subunit that regulates AKT-1 dephosphorylation (Padmanabhan et al., 2009). PPTR-1 binds to AKT-1 and reduces AKT-1 phosphorylation at the site corresponding to T308 in human Akt1 in vivo. pptr-1 knockdown suppresses dauer arrest, reduces thermotolerance, and shortens the life span of $d a f-2 / I G F R$ mutants, suggesting that PPTR-1 antagonizes IIS. pptr-1 knockdown also promotes the translocation of nuclear DAF-16/FoxO to the cytoplasm, presumably by increasing AKT-1 phosphorylation and activation (Padmanabhan et al., 2009). PPTR-1 homologs also modulate Akt activity in mammalian cells and Drosophila (Vereshchagina et al., 2008; Padmanabhan et al., 2009), indicating that PP2A regulation of Akt activity is evolutionarily conserved.

\subsection{SGK-1}

The serum- and glucocorticoid-inducible kinase Sgk is also activated by growth factors in a PI3K-dependent manner and is similar to Akt/PKB in amino acid sequence as well as in substrate specificity (Bruhn et al., 2010; Pearce et al., 2010). In mammalian cells, both Sgk and Akt/PKB can phosphorylate FoxO3A, leading to its inhibition through cytoplasmic sequestration (Brunet et al., 2001). Accordingly, the C. elegans Sgk ortholog SGK-1 can phosphorylate DAF-16/FoxO in vitro (Hertweck et al., 2004). In C. elegans, knockdown of SGK-1 by RNAi induces nuclear translocation of DAF-16/FoxO and extends life span in a DAF-16/FoxO-dependent manner, suggesting that SGK-1 may function similarly to AKT-1 and AKT-2 in life span control (Hertweck et al., 2004).

However, recent studies have revealed that $s g k-1$ null mutants have a shorter life span than wild-type animals (Soukas et al., 2009; Alam et al., 2010; Kwon et al., 2010). Furthermore, an $s g k$ - 1 gain-of-function mutation extends life span in a DAF-16/FoxO-dependent manner when animals are incubated at $20^{\circ} \mathrm{C}$, suggesting that SGK-1 promotes longevity (Chen et al., 2013a). Notably, in contrast to akt-1 null mutation, which increases the expression of DAF-16/FoxO target genes by promoting DAF-16/FoxO nuclear translocation (Zhang et al., 2008; Alam et al., 2010; Dumas et al., 2010), sgk-1 null and gain-of-function mutations did not influence DAF-16/FoxO subcellular localization and had different effects on the expression of distinct DAF-16/FoxO target genes (Chen et al., 2013a). These data underscore fundamental differences in DAF-16/FoxO regulation by AKT-1 and SGK-1. Whereas AKT-1 shortens life span by inhibiting DAF-16/FoxO through direct phosphorylation, SGK-1 extends life span, possibly through indirect regulation of a subset of DAF-16/FoxO targets. Further studies will be necessary to resolve the discrepancy between the effects of $s g k-1$ mutation and $s g k-1$ RNAi on life span (Hertweck et al., 2004; Chen et al., 2013a).

\subsection{4-3-3 proteins}

In mammalian cells, phosphorylation of FoxO3a by Akt promotes its retention in the cytoplasm and binding to 14-3-3 proteins (Brunet et al., 1999). During larval development, the $C$. elegans 14-3-3 proteins PAR-5 and FTT-2 sequester DAF-16/FoxO in the cytoplasm, as knockdown of either par-5 or $\mathrm{ftt}-2$ promotes translocation of a functional DAF-16::GFP fusion protein to the nucleus (Berdichevsky et al., 2006; Li et al., 2007a). ftt-2 RNAi also enhances dauer arrest in animals with reduced IIS (Li et al., 2007a), indicating that FTT-2 inhibits DAF-16/FoxO action. The role of 14-3-3 proteins in the regulation of DAF-16/FoxO activity in adulthood is more complex. FTT-2 inhibits DAF-16/FoxO target gene expression in young adults ( $\mathrm{Li}$ et al., 2007a), in accord with its effect on dauer arrest and DAF-16::GFP subcellular localization in larvae (Berdichevsky et al., 2006; Li et al., 2007a). Paradoxically, FTT-2 promotes life span extension in a SIR-2.1- and DAF-16/FoxO-dependent manner (Berdichevsky et al., 2006; Wang et al., 2006). 


\section{The FoxO transcription factor DAF-16}

daf-16/FoxO null mutations fully suppress most phenotypes associated with reduced IIS, suggesting that these phenotypes are a consequence of increased DAF-16/FoxO activity. Accordingly, DAF-16/FoxO is likely the primary mediator of the dauer-constitutive, increased longevity, and stress resistance phenotypes observed in mutants with reduced IIS. DAF-16 is a member of the FoxO family of Forkhead transcription factors, which are key regulators of growth, metabolism, stress responses, cell cycle control, and longevity in many organisms (Accili and Arden, 2004). The Forkhead DNA binding domain, DNA binding specificity, RxRxxS/T phosphorylation motifs, and 14-3-3 binding sites are conserved between $C$. elegans DAF-16 and mammalian FoxOs (Obsil and Obsilova, 2008). DAF-16/FoxO is inhibited by the IIS pathway through phosphorylation at its RxRxxS/T motifs, which results in its cytoplasmic retention (Lee et al., 2001; Lin et al., 2001). Mutation of daf-2/IGFR, age-1/PI3K, pdk-1 or akt-1/2 reduces DAF-16/FoxO phosphorylation, resulting in its nuclear translocation and subsequent transcriptional regulation of DAF-16/FoxO target genes (see Section 5.4) that results in IIS loss-of-function phenotypes (see Section 3).

\subsection{DAF-16/FoxO isoforms}

The daf-16/FoxO genomic locus encodes eight distinct transcripts. Two of these, daf-16e and daf-16g, are predicted to encode proteins that lack a DNA binding domain; the functions of these isoforms are not known. The remaining six mRNAs comprise three groups of transcripts known as daf-16a, daf-16b, and daf-16d/f/h that are transcribed from three distinct promoters (Figure 2A) and that encode proteins with different N-termini (Lin et al., 1997; Ogg et al., 1997; Kwon et al., 2010) (Figure 2B).

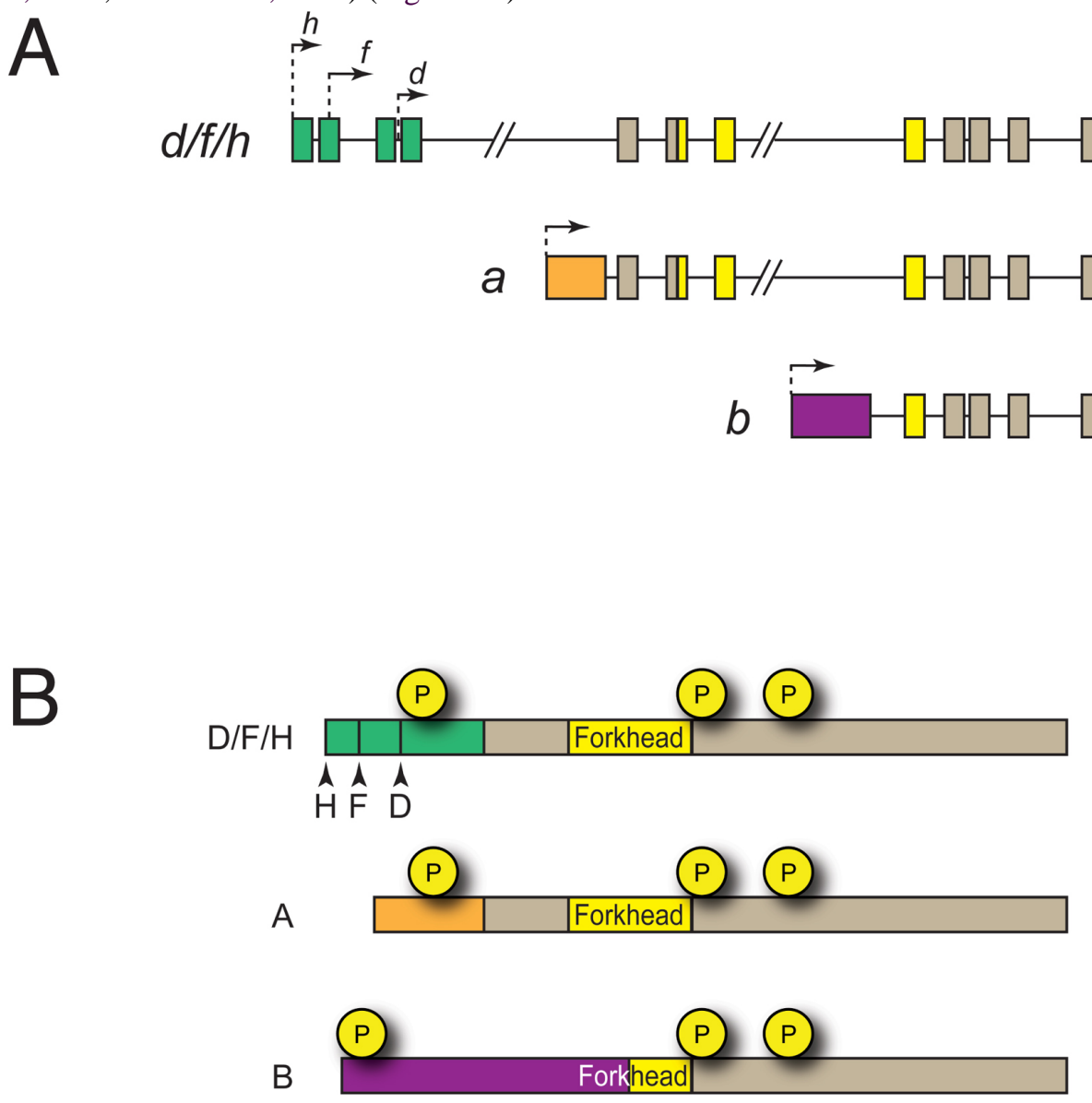

Figure 2. daf-16/FoxO isoforms. A. Genomic structure of the $d a f-16 / F o x O$ locus (not drawn to scale). $a, b$, and $d / f / h$ isoforms and their promoters are depicted. Isoform-specific exons are colored green, orange, and purple. B. DAF-16/FoxO polypeptides. Amino acid sequences encoded by isoform-specific exons are colored green, orange, and purple. All isoforms share two conserved Akt-dependent phosphorylation sites that lie carboxyterminal to the Forkhead DNA binding domain but have unique sites in their aminotermini. See text for details. 
Transcriptional and translational reporters indicate that daf-16a, daf-16b, and $d a f-16 d / f / h$ have distinct tissue distributions. daf-16a and $d a f-16 d / f / h$ promoters are active in most tissues, driving reporter gene expression in hypodermis, muscle, neurons, and intestine (Ogg et al., 1997; Henderson and Johnson, 2001; Lee et al., 2001; Lin et al., 2001; Kwon et al., 2010). By contrast, the daf-16b promoter exhibits greater tissue specificity, driving expression primarily in the pharynx, nervous system, and somatic gonad (Lee et al., 2001; Kwon et al., 2010; Christensen et al., 2011). Promoter-swap experiments with isoform-specific daf-16/FoxO transgenes indicate that promoter specificity contributes to the distinct biological functions of specific daf-16/FoxO isoforms (Lee et al., 2001; Kwon et al., 2010).

The three groups of DAF-16/FoxO isoforms share carboxy-terminal sequences but diverge at their amino-termini (Figure 2B). Human FoxO1, FoxO3, and FoxO4 proteins are most similar in sequence to DAF-16A and DAF-16D/F/H, which share their 428 carboxy-terminal amino acids (including the entire Forkhead DNA binding domain) (Kwon et al., 2010). Importantly, whereas all three RxRxxS/T motifs in mammalian FoxO transcription factors that are phosphorylated in response to Akt/PKB activation are conserved in DAF-16A, the divergent amino-terminus of DAF-16D/F/H lacks the amino-terminal RxRxxT motif (Kwon et al., 2010). Instead, DAF-16D/F/H isoforms are phosphorylated in vivo at an amino-terminal site within a QxRxxS motif (Bodenmiller et al., 2008; Kwon et al., 2010). Since the -5 arginine in the canonical RxRxxS/T motif of the Akt/PKB substrate GSK3 makes important molecular contacts with conserved acidic residues in the kinase domain of Akt/PKB and other highly related kinases (Yang et al., 2002), the QxRxxS motif in DAF-16D/F/H and the amino-terminal RxRxxT motif in DAF-16A may be phosphorylated and regulated by distinct kinases.

The 319 C-terminal amino acids of DAF-16B, which include part of but not the entire Forkhead domain, are identical to those of DAF-16A and DAF-16D/F/H. The amino-terminal 211 amino acids of DAF-16B, including the amino-terminal portion of its Forkhead domain, are encoded by a unique exon (Ogg et al., 1997). The fact that the DAF-16B DNA binding domain differs in amino acid sequence from that of DAF-16A and DAF-16D/F/H suggests that it may bind to DNA sequences distinct from those recognized by DAF-16A and DAF-16D/F/H. However, a $d a f-16 b$ cDNA expressed under the control of the daf-16a promoter extends the life span of daf-16/FoxO null mutant animals to nearly the same degree as daf-16a expressed from the daf-16a promoter, and expression of either cDNA under the control of the $d a f-16 b$ promoter suffices to induce pharyngeal remodeling in dauers (Lee et al., 2001). Thus, DAF-16A and DAF-16B may have similar functions in spite of some amino acid sequence divergence in their Forkhead domains. Although the amino-terminus of DAF-16B is unique, it does contain an RxRxxT motif, suggesting that DAF-16A and DAF-16B may be regulated by the same or similar protein kinases.

\subsection{Functions of specific DAF-16/FoxO isoforms}

daf-16/Fox $O$ null mutations and mutations predicted to only affect $d a f-16 a$ and $d a f-16 d / f / h$ isoforms suppress the dauer-constitutive and extended life span phenotypes of daf-2/IGFR mutants to similar degrees (Lee et al., 2001), and RNAi knockdown of $d a f-16 a$ and $d a f-16 d / f / h$ together suppresses most of the life span extension caused by $d a f-2 / I G F R$ mutation (Kwon et al., 2010). Furthermore, expression of $d a f-16 a$-specific and $d a f-16 d / f / h$-specific transgenes suffices to rescue dauer-constitutive and longevity phenotypes in daf-16(null); daf-2(e1370) double mutants (Lin et al., 2001; Kwon et al., 2010). By contrast, daf-16b-specific transgenes have a small effect on longevity and dauer arrest (Lee et al., 2001; Kwon et al., 2010). Taken together, these results suggest that DAF-16A and DAF-16D/F/H are the major isoforms that control dauer arrest and life span. Studies with isoform-specific transgenes suggest that AKT-1 influences life span primarily by controlling DAF-16D/F/H activity, whereas AKT-2 controls life span mainly by regulating DAF-16A (Kwon et al., 2010). A recent study examining the contributions of specific mTOR complexes to life span control revealed that $C$. elegans TORC1 controls life span by regulating the subcellular localization of DAF-16D/F/H without influencing DAF-16A activity (Robida-Stubbs et al., 2012). Thus, a picture is emerging whereby specific DAF-16/FoxO isoforms influence life span by responding to distinct regulatory inputs. The unique transcriptional outputs that may distinguish the biological activities of the different isoforms are not yet known.

No $d a f-16 b$-specific mutations have been reported, and the observation that $d a f-16 b$ is mainly expressed in tissues that may be refractory to RNAi, such as the pharynx and the nervous system, complicates the interpretation of negative $d a f-16 b$ RNAi results. Expression of daf-16a transgenes under the control of the daf-16a promoter in daf-16/FoxO null mutants is sufficient to rescue the dauer-defective phenotype; however, dauer formation in these animals is incomplete, as these dauers do not have fully remodeled pharynxes that are typically seen in natural dauers (Henderson and Johnson, 2001; Lee et al., 2001; Lin et al., 2001; Kwon et al., 2010). Expression of a daf-16b transgene under the control of its native promoter is sufficient for partial rescue of the dauer-defective phenotype of 
daf-16/FoxO null mutants, and dauers formed in this context have fully remodeled pharynxes (Lee et al., 2001; Kwon et al., 2010). Therefore, DAF-16B plays an important role in pharyngeal remodeling during dauer formation. A recent study also implicates DAF-16B in promoting neurite outgrowth cell autonomously in the AIY interneuron (Christensen et al., 2011).

\subsection{The DAF-16 Binding Element (DBE)}

The DNA binding domain of DAF-16/FoxO, like that of other members of the FoxO transcription factor family, binds in vitro to a core consensus DNA sequence, TTGTTTAC, known as the DBE (DAF-16 Binding Element), as shown through in vitro SELEX studies (Furuyama et al., 2000). Variations of the DBE are overrepresented in the promoters of genes that are upregulated in daf-2/IGFR mutants in a DAF-16/FoxO-dependent manner (Class 1 DAF-16/FoxO targets) (Murphy et al., 2003), and recent DamID (chromatin profiling by DNA adenine methyltransferase identification) experiments suggest that DAF-16/FoxO binds directly to these target genes (Schuster et al., 2010).

By contrast, DAF-16/FoxO appears not to bind the promoters of genes that are downregulated in daf-2/IGFR mutants in a DAF-16/FoxO-dependent manner (Class 2 DAF-16/FoxO targets). These promoters contain a different overrepresented sequence, CTTATCA (Murphy et al., 2003), a reverse GATA site that was later dubbed the "DAE" for "DAF-16 Associated Element" (McElwee et al., 2004). Given its lack of similarity with the DBE, it seems unlikely that FoxO transcription factors would bind to the DAE; thus a transcription factor distinct from DAF-16/FoxO may be involved in the regulation of Class 2 (downregulated) DAF-16/FoxO targets. In fact, ChIP-Seq data implicates the zinc finger transcription factor PQM-1 as the DAE-binding factor, acting antagonistically with DAF-16/FoxO (Tepper et al., 2013) (Section 6.12).

\subsection{Transcriptional targets of IIS}

DAF-16/FoxO is the main output of IIS. While the regulation of many DAF-16/FoxO target genes in daf-2/IGFR mutants was already known from individual studies of specific stresses, the power of whole-genome techniques, including bioinformatic predictions (Lee et al., 2003), expression microarrays (McElwee et al., 2003; Murphy et al., 2003; McElwee et al., 2004; Shaw et al., 2007), serial analysis of gene expression (SAGE) (Halaschek-Wiener et al., 2005), protein mass spectrometry (Dong et al., 2007), DamID (Schuster et al., 2010), and ChIP-Seq (Niu et al., 2011; Tepper et al., 2013), have revealed a large complement of DAF-16/FoxO targets (discussed in greater detail in Murphy, 2006). While the functions of many DAF-16/FoxO targets remain obscure, the diverse array of characteristics of known DAF-16/FoxO-regulated genes helps explain why daf-2/IGFR mutant animals are resistant to virtually every known stress they might encounter.

DAF-16/FoxO upregulates the expression of many genes whose protein products fight various forms of stress, such as oxidative stress through superoxide dismutase (sod-3) (Honda and Honda, 1999, 2002; Oh et al., 2006) and catalase (ctl-1, 2) (Yanase et al., 2002); resistance to heavy metals through the $m t l-1$ metallothionein (Barsyte et al., 2001); thermotolerance and proteotoxicity via small heat shock proteins (hsp-16, hsp-12.6) (Hsu et al., 2003; Morley and Morimoto, 2004; Cohen et al., 2006; David et al., 2010; Keowkase et al., 2010; Ching et al., 2011; Teixeira-Castro et al., 2011; Zhang et al., 2012); hypertonic stress through trehalose biosynthetic enzymes (Murphy et al., 2003; Lamitina and Strange, 2005); mitochondrial oxidative stress (Arkblad et al., 2005) via the nnt-1 nicotinamide nucleotide transhydrogenase (Shaw et al., 2007; McCormick et al., 2011); and antimicrobial defense through thaumatins (PR5 plant defense proteins) and a variety of bacterial pathogen defense genes (spp-1, lys-7, DUF-23, C-lectins, scl- genes, ShK toxins, etc.) (Murphy et al., 2003; Ookuma et al., 2003; Evans et al., 2008).

In addition to stress response genes, reduction of $d a f-2 / I G F R$ activity results in increased expression of metabolic regulators, such as gei-7 (isocitrate lyase/malate synthase, which is also upregulated in dauers) (Wang and Kim, 2003), the dod-9 acyl CoA synthetase, gpd-2 glyceraldehyde 3-phosphate dehydrogenase, and ges-1 carboxylesterase; digestive proteins, such as the GCP-1 cysteine protease; fat metabolism genes, including fatty acid desaturase (fat-7), alcohol dehydrogenases (sodh-1/dod-11, sodh-2/dod-14) and short-chain acyl-CoA dehydrogenases (acdh-1/dod-12) (Murphy et al., 2003); and many cytochrome P450s (cyp-13A4/dod-1, cyp-35B1/dod-13, cyp-34A9/dod-16), some of which may function in Phase II detoxification (McElwee et al., 2004; Oliveira et al., 2009), and others that may function in the synthesis of sterol signaling ligands, similar to DAF-9 (Gerisch et al., 2001; Jia et al., 2002; Murphy et al., 2003). Autophagy is also controlled by daf-2/IGFR through its regulation of bec- 1 expression (Melendez et al., 2003). Still other genes upregulated in daf-2/IGFR mutants, such as the hil-1 histone H1-like gene (Shaw et al., 2007), are required for their long life span, but their functions are not yet well understood. 
Conversely, "Class 2" genes are downregulated in daf-2/IGFR mutants in a DAF-16/FoxO-dependent manner (Murphy et al., 2003). The functions of most of these genes and their roles in normal development and life span control are not yet understood, but recent GO analysis suggests that many of these genes regulate growth and development (Tepper et al., 2013), and reducing expression of some of these genes by RNAi extends life span (Murphy et al., 2003). Many Class 2 genes are upregulated in response to pathogen exposure (Troemel et al., 2006), suggesting a specific role in pathogen response that may normally "cost" longevity, but this has not yet been shown. Most of the Class 2 genes, and some of the Class 1 genes, are regulated by the zinc finger transcription factor PQM-1, which acts in opposition to DAF-16/FoxO (Tepper et al., 2013) (Section 6.12).

The regulation of Class 1 and Class 2 gene expression by DAF-16/FoxO may function in part to coordinate the state of IIS at the organismal level. When IIS is reduced, DAF-16/FoxO represses the Class 2 ILP gene ins-7 (which encodes an agonist of DAF-2/IGFR) and promotes the expression of the Class 1 ILP gene ins-18 (which encodes a DAF-2/IGFR antagonist), resulting in feed-forward inhibition of IIS throughout the animal (Murphy et al., 2003; Murphy et al., 2007).

\section{Other DAF-16/FoxO co-factors and regulators}

DAF-16/FoxO is regulated by a host of additional proteins that directly or indirectly modulate its activity (Figure 3) (Landis and Murphy, 2010). Like the IIS pathway, DAF-16/FoxO co-factors and regulators and the nature of their interactions with DAF-16/FoxO are remarkably conserved throughout metazoan phylogeny.

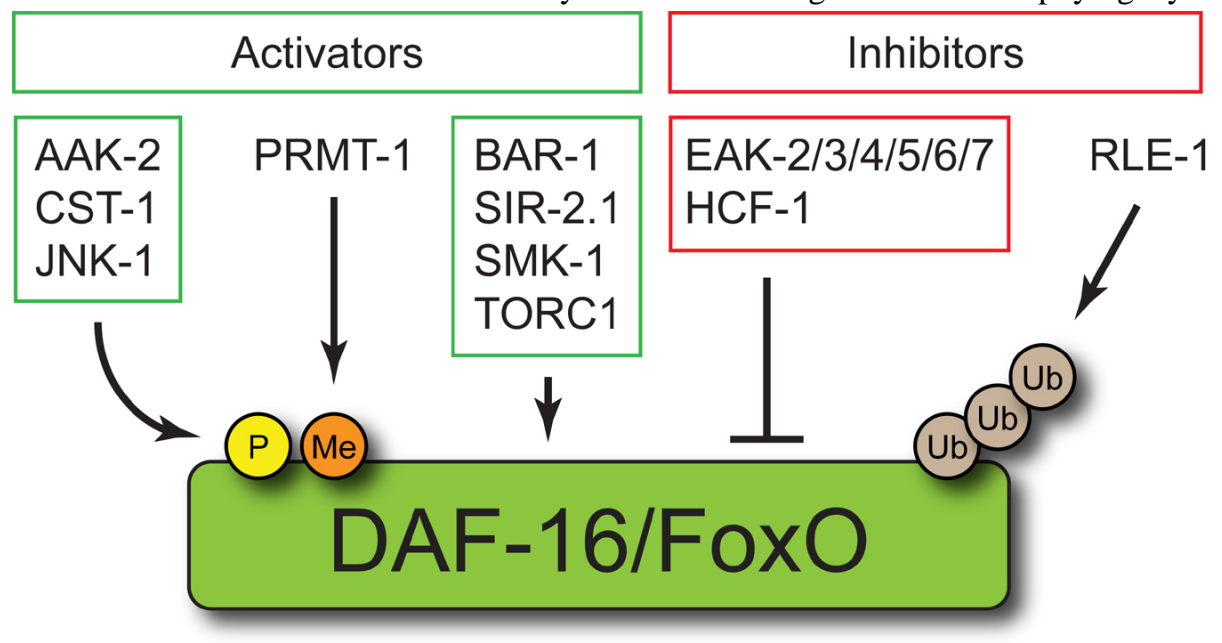

Figure 3. DAF-16/FoxO regulators and cofactors. DAF-16/FoxO is directly regulated by the serine/threonine kinases AAK-2, CST-1, and JNK-1, the arginine methyltransferase PRMT-1, and the E3 ubiquitin ligase RLE-1. The mechanistic basis for DAF-16/FoxO regulation by BAR-1, SIR-2.1, SMK-1, TORC1, the EAK proteins, and HCF-1 has not been established. See text for details.

\subsection{DAF-16/FoxO regulation by kinases other than Akt}

While IIS-regulated DAF-16/FoxO phosphorylation by AKT-1 and AKT-2 at conserved RxRxxS/T motifs promotes the cytoplasmic sequestration of DAF-16/FoxO, phosphorylation of DAF-16/FoxO by other kinases may also regulate DAF-16/FoxO activity. For example, the mammalian MST1 Ste20-like kinase activates FoxO3 by phosphorylating it at a conserved residue within its DNA binding domain (S207), thus promoting its nuclear translocation by disrupting interactions with 14-3-3 proteins. Mammalian MST1 can also phosphorylate C. elegans DAF-16/FoxO at the site corresponding to S207 in FoxO3 (S196). Overexpression of the C. elegans MST-1 homolog CST-1 extends life span in a DAF-16/FoxO-dependent manner, suggesting that MST1 activates FoxO transcription factors via a conserved mechanism (Lehtinen, 2006). However, phosphorylation of the FoxO1 DNA binding domain by MST1 inhibits its binding to cognate DNA sites in vitro (Brent et al., 2008). Therefore, our understanding of how MST1 regulates FoxO activity appears to be incomplete.

In mammalian cells, oxidative stress activates FoxO4 through c-Jun N-terminal kinase (Jnk)-dependent phosphorylation of two threonine residues near its C-terminus (T447 and T451) (Essers et al., 2004). Jnk also activates FoxO in invertebrates, as Jnk orthologs promote life span extension in a FoxO-dependent manner in $C$. elegans and Drosophila (Oh et al., 2005; Wang et al., 2005). However, whether the underlying mechanisms of FoxO 
activation by Jnk are conserved is unclear; whereas Jnk phosphorylation sites lie near the C-terminus of mammalian FoxO4 (Essers et al., 2004), C. elegans JNK-1 appears to phosphorylate residues in an N-terminal fragment of DAF-16/FoxO in vitro (Oh et al., 2005).

The AMP-dependent protein kinase (AMPK) controls energy balance by sensing changes in intracellular AMP/ATP ratios (Hardie et al., 2012). In C. elegans the AMPK catalytic subunit homolog AAK-2 promotes longevity and stress resistance (Apfeld et al., 2004; Greer et al., 2007a; Lee et al., 2008; Narbonne and Roy, 2009; LaRue and Padilla, 2011; Mair et al., 2011). This may be a consequence of DAF-16/FoxO activation, as an activating mutation in aakg-2, which encodes a C. elegans homolog of the AMPK gamma subunit, enhances resistance to paraquat and extends life span in a DAF-16/FoxO-dependent manner (Greer et al., 2007a). AAKG-2 activation increases DAF-16/FoxO target gene expression without influencing DAF-16/FoxO subcellular localization, suggesting that it regulates DAF-16/FoxO activity independent of the PI3K/Akt pathway (Greer et al., 2007a). Activated AMPK can phosphorylate both DAF-16/FoxO and human FoxO3 at several sites in vitro, resulting in the enhancement of FoxO-dependent transcription in mammalian cells (Greer et al., 2007a; Greer et al., 2007b). The role of AMPK in controlling FoxO activity in vivo is likely to be context-dependent, as AMPK can inhibit FoxO transcription factor activity indirectly in mouse hepatocytes through direct effects on Class IIa histone deacetylases (Mihaylova et al., 2011).

\subsection{EAK proteins}

Although Akt inhibition of FoxO transcription factors by direct phosphorylation is well established as a conserved mechanism of FoxO regulation by IIS, data from mammalian cell culture as well as C. elegans experiments suggest that nuclear translocation of FoxO is necessary but not sufficient for full activation of FoxO. For example, in mammalian cells, insulin inhibits transcriptional activation by a FoxO1 mutant that is not exported from the nucleus (Tsai et al., 2003). Similarly, a DAF-16/FoxO mutant with all RxRxxS/T phosphoacceptor sites mutated to alanine localizes to nuclei constitutively but does not promote dauer arrest or extend life span unless IIS is reduced (Lin et al., 2001). These data are consistent with the existence of a conserved pathway that acts in parallel to Akt to inhibit FoxO activity after its translocation to the nucleus.

A genetic screen for enhancers of the weak dauer-constitutive phenotype of $\underline{a k t}-1$ null mutants (eak mutants) identified six genes that may encode components of such a pathway (Hu et al., 2006; Zhang et al., 2008; Alam et al., 2010; Dumas et al., 2010). Two eak genes, eak-2 and eak-5, are respectively allelic to $h s d-1$ and $s d f-9$, genes identified in unrelated synthetic dauer screens (Ohkura et al., 2003; Patel et al., 2008a). In contrast to AKT-1, which inhibits DAF-16/FoxO by promoting its cytoplasmic sequestration, EAK proteins inhibit DAF-16/FoxO activity without influencing its subcellular localization (Zhang et al., 2008; Alam et al., 2010; Dumas et al., 2010). hsd-1 and eak-7 null mutations both enhance the dauer arrest phenotype of animals expressing a constitutively nuclear DAF-16/FoxO mutant protein, suggesting that HSD-1 and EAK-7 inhibit nuclear DAF-16/FoxO (Alam et al., 2010).

The EAK proteins may control nuclear DAF-16/FoxO activity in part by influencing the activity of DAF-12, a conserved nuclear receptor that is regulated by steroid ligands known as dafachronic acids (Antebi et al., 2000; Motola et al., 2006; WormBook chapter Dauer). Five EAK proteins, including the conserved 3- $\beta$-hydroxysteroid dehydrogenase family member HSD-1, are specifically expressed in the hypodermal XXX cells (Hu et al., 2006; Patel et al., 2008a; Zhang et al., 2008; Dumas et al., 2010), where the DA biosynthetic enzyme DAF-9 is also expressed (Gerisch et al., 2001; Jia et al., 2002; Ohkura et al., 2003). daf-9 and daf-36 mutations, which abrogate $\Delta^{7}$-DA biosynthesis (Motola et al., 2006; Wollam et al., 2011), also enhance dauer arrest in animals with reduced akt-1 activity (Zhang et al., 2008; Dumas et al., 2010). Furthermore, DAF-12 is required for the expression of DAF-16/FoxO target genes in eak; akt-1 double mutants as well as in daf-36 mutants with reduced akt-1 activity (Zhang et al., 2008; Dumas et al., 2010). Taken together, these data are consistent with a model whereby EAK proteins act in the XXX cells to promote DA biosynthesis and/or release. DAs then reduce DAF-16/FoxO activity in target tissues by binding to and regulating the activity of DAF- 12 .

In contrast to the other five eak genes, which are specifically expressed in the XXX cells, the conserved gene eak-7 is expressed in the same tissues as daf-16/FoxO. EAK-7 and its human ortholog KIAA1609 both contain $\mathrm{N}$-myristoylation motifs and a TLDc domain of unknown function (Alam et al., 2010). How EAK-7 controls nuclear DAF-16/FoxO activity is not known. Since EAK-7 localizes to the plasma membrane (Alam et al., 2010), it likely acts through intermediary proteins to regulate DAF-16/FoxO in the nucleus. 


\subsection{E3 ubiquitin ligases}

In C. elegans, the RLE-1 ubiquitin ligase controls dauer arrest and life span by promoting the polyubiquitination and proteasomal degradation of DAF-16/FoxO ( $\mathrm{Li}$ et al., 2007b). Interestingly, although a number of E3 ubiquitin ligases have been shown to promote FoxO polyubiquitination and degradation in mammalian cell culture, the RLE-1 ortholog Roquin is not one of them (Matsuzaki et al., 2003; Huang et al., 2005; Kato et al., 2008; Fu et al., 2009). This suggests that distinct E3 ligases may be capable of regulating FoxO turnover in different biological contexts. E3 ligases can also regulate DAF-16/FoxO indirectly: components of an SCF E3 ligase complex containing the cullin CUL-1 are required for life span extension in daf-2/IGFR mutants, and CUL-1 promotes DAF-16/FoxO target gene expression (Ghazi et al., 2007).

\subsection{PRMT-1}

Mammalian FoxO1 is regulated by arginine methylation. The arginine methyltransferase PRMT1 methylates FoxO1 at arginines 248 and 250, which comprise part of the RxRxxS motif that is phosphorylated by Akt/PKB. Methylation of R248 and R250 activates FoxO1 by preventing its phosphorylation by Akt/PKB (Yamagata et al., 2008). This mechanism of FoxO regulation is conserved in C. elegans, as the PRMT1 ortholog PRMT- 1 binds to and methylates DAF-16/FoxO, thus reducing T242 phosphorylation (analogous to S253 in mammalian FoxO1) and promoting DAF-16/FoxO nuclear translocation, longevity, and stress resistance (Takahashi et al., 2011). Surprisingly, whereas prmt-1 mutation shortens life span and reduces stress resistance of daf-2(e1368) mutant animals, it does not suppress the dauer-constitutive phenotype of daf-2(e1368) (Takahashi et al., 2011).

\subsection{BAR-1/ $\beta$-catenin}

$\beta$-catenin has a conserved function as a FoxO regulator. A C. elegans $\beta$-catenin, BAR-1, promotes DAF-16/FoxO activity in response to oxidative stress. bar-1 mutation reduced expression of a DAF-16/FoxO-dependent reporter gene in the presence of paraquat, but not through the canonical $\beta$-catenin/TCF pathway. bar-1 is also required for dauer arrest in daf-2(m41) mutants. BAR-1 physically interacts with DAF-16/FoxO when both proteins are expressed in mammalian cell culture, and this interaction is enhanced in the presence of oxidative stress. Accordingly, mammalian $\beta$-catenin binds to FoxO transcription factors directly and promotes transcription from a FoxO-dependent promoter, supporting a conserved role for $\beta$-catenin in FOXO activation (Essers et al., 2005).

\subsection{Sirtuins}

A role for the sirtuin family of $\mathrm{NAD}^{+}$-dependent deacetylases in FoxO regulation was first suggested by studies in $C$. elegans showing that increased dosage of the sir-2.1 gene extends life span in a DAF-16/FoxO-dependent manner (Tissenbaum and Guarente, 2001). The longevity increase caused by sir-2.1 overexpression in invertebrates may be milder than originally reported-15\% (Tissenbaum and Guarente, 2001; Burnett et al., 2011; Viswanathan and Guarente, 2011), rather than the 50\% originally reported (Tissenbaum and Guarente, 2001; Burnett et al., 2011; Viswanathan and Guarente, 2011). A low-copy sir-2.1 overexpression strain is long-lived in a daf-16/FoxO-dependent manner, and its longevity phenotype is suppressed by sir-2.1 RNAi (Rizki et al., 2011). Additionally, sirtuins interact physically with FoxO in vivo in C. elegans (Berdichevsky et al., 2006) and in intact mammalian cells (Brunet et al., 2004; Daitoku et al., 2004; Motta et al., 2004; van der Horst et al., 2004). In response to heat shock, the physical association of SIR-2.1 and DAF-16/FoxO requires 14-3-3 proteins (Berdichevsky et al., 2006). These results suggest that SIR-2.1 may control the acetylation state of DAF-16/FoxO in a manner analogous to the regulation of mammalian FoxO transcription factors by SIRT1 (Brunet et al., 2004; Motta et al., 2004; van der Horst et al., 2004).

Recently, Chiang et al. characterized the C. elegans homolog of mammalian SIRT6 and SIRT7 proteins, SIR-2.4, and found that it is required for nuclear localization of DAF-16/FoxO in response to stress but dispensable for DAF-16/FoxO nuclear localization in the context of reduced IIS. Although DAF-16/FoxO is hyperacetylated in sir-2.4 mutants, a catalytically inactive mutant SIR-2.4 protein rescued the DAF-16/FoxO localization defect caused by sir-2.4 mutation, suggesting that SIR-2.4 controls the acetylation state of DAF-16/FoxO indirectly (Chiang et al., 2012b). 


\section{7. p300/CBP-1}

CBP-1, the $C$. elegans ortholog of the mammalian CBP/p300 acetyltransferase, is ubiquitously expressed, and regulates DAF-16/FoxO acetylation. CBP/p300 acetylates mammalian FoxO1 (Daitoku et al., 2004), and C. elegans CBP-1 interacts with DAF-16/FoxO in yeast two-hybrid assays and in vitro (Nasrin et al., 2000). More recently, it was shown that RNAi knockdown of $c b p-1$ results in DAF-16/FoxO hypoacetylation and nuclear translocation (Chiang et al., 2012b). CBP-1 acetylates DAF-16/FoxO at four sites in vitro (K248, K253, K375, and K379), and these acetylation events are blocked by SIR-2.4 in a manner that is independent of SIR-2.4 catalytic activity (Chiang et al., 2012b).

\subsection{HCF-1/host cell factor}

HCF-1 is the $C$. elegans ortholog of mammalian host cell factor, which is a nuclear protein that associates with the herpes simplex virus transcriptional activator VP16 to promote early viral gene expression during lytic infection (Wilson et al., 1993). $h c f-1$ mutation extends life span and enhances stress resistance in a DAF-16/FoxO-dependent manner. HCF-1 is a nuclear protein that binds to DAF-16/FoxO and inhibits its activity by preventing its association with target gene promoters (Li et al., 2008). Interestingly, SIR-2.1 may control DAF-16/FoxO activity via HCF-1, as SIR-2.1 overexpression does not enhance stress resistance or extend life span in animals lacking HCF-1. Moreover, expression profiling experiments demonstrate significant overlap in the sets of genes regulated by SIR-2.1 overexpression and loss of HCF-1, and HCF-1 physically associates with SIR-2.1 (Rizki et al., 2011). Mammalian host cell factors also regulate FoxO target gene expression and form a complex with FoxO3 and SIRT1, suggesting that the role of host cell factors in mediating sirtuin control of FoxO transcription factor activity may be evolutionarily conserved (Li et al., 2008; Rizki et al., 2011).

\subsection{SMK-1}

SMK-1, which is orthologous to the mammalian protein phosphatase 4 regulatory subunit 3A, was originally identified in a screen in Dictyostelium for suppressors of the MEK1 null phenotype (Mendoza et al., 2005). $C$. elegans SMK-1 is a nuclear protein that is expressed in the intestine, hypodermis, and nervous system. It is required for longevity in IIS loss-of-function mutants but does not influence dauer arrest (Wolff et al., 2006). Interestingly, SMK-1 promotes resistance to oxidative stress, ultraviolet radiation, and pathogens but does not appear to influence thermotolerance or reproduction in IIS mutants (Wolff et al., 2006). These data suggest that SMK-1 regulates a subset of DAF-16/FoxO functions. Although SMK-1 co-localizes and genetically interacts with DAF-16/FoxO, how it controls DAF-16/FoxO activity [specifically, whether it promotes the dephosphorylation of DAF-16/FoxO, and if so, at which site(s)] remains unclear. Furthermore, the full complement of DAF-16/FoxO targets that are regulated by SMK-1 is not known.

\subsection{DPY-21}

A genetic screen designed to identify new DAF-16/FoxO regulators that promote dauer arrest has revealed an unexpected role for the conserved dosage compensation protein DPY-21 in the regulation of IIS and DAF-16/FoxO activity. Three independent $d p y-21$ alleles emerged from a screen for suppressors of the eak-7; akt-1 dauer-constitutive phenotype. DPY-21 is a component of the C. elegans dosage compensation complex (DCC), a ten-protein complex that equalizes X-linked gene expression between XO males and XX hermaphrodites by binding to both hermaphrodite $\mathrm{X}$ chromosomes and reducing gene expression from each by approximately two-fold (see X-Chromosome dosage compensation; Meyer, 2010). DPY-21 activates DAF-16/FoxO by repressing X-linked genes encoding IIS components that promote the cytoplasmic sequestration of DAF-16/FoxO; accordingly, $d p y-21$ knockdown reduces DAF-16/FoxO target gene expression and enhances DAF-16/FoxO translocation to the cytoplasm (Dumas et al., 2013a).

\subsection{TRPA-1}

The cold-sensitive TRP channel TRPA-1 was recently shown to promote longevity at low temperatures $\left(15-20^{\circ} \mathrm{C}\right)$ in a DAF-16/FoxO-dependent manner by activating a calcium-dependent kinase cascade involving the calcium-sensitive protein kinase $\mathrm{C}$ family member PKC-2 and SGK-1. Although life span extension induced by TRPA-1 activation required DAF-16/FoxO, TRPA-1 activation did not promote DAF-16/FoxO nuclear localization, suggesting that TRPA-1 extends life span by activating nuclear DAF-16/FoxO (Xiao et al., 2013). 


\subsection{2. $P Q M-1$}

The identification of the DAF-16 Associated Element as an overrepresented motif in the promoters of Class 2 (daf-2-downregulated) genes suggested that another factor might collaborate with DAF-16/FoxO in IIS-dependent gene regulation. By combining a ranking of genes by DAF-16/FoxO responsiveness with ChIP-Seq analysis, Tepper, et al. (2013) identified the zinc finger transcription factor PQM-1 as a candidate DAE-binding factor. PQM-1 binds to a Class 2 gene promoter in a DAE-dependent manner, and expression is reduced in the context of reduced IIS, as predicted for a Class 2 gene. More interestingly, PQM-1 and DAF-16/FoxO occupy the nucleus in a mutually exclusive and IIS-dependent manner. When IIS is intact, PQM-1 resides in the nucleus, activating growth and development genes, while DAF-16/FoxO is in the cytoplasm. Under stress or conditions of reduced IIS, DAF-16/FoxO enters the nucleus, inducing the expression of stress-response genes, and PQM-1 is primarily cytoplasmic. While PQM-1 is regulated by IIS, DAF-16 and PQM-1 also have an influence on the nuclear localization of one another, as the loss of each increases the nuclear occupancy of the other (Tepper et al., 2013).

\subsection{FoxO regulators in other organisms}

The conserved gene Melted was identified in a gain-of-function screen for genes that affect cell growth in Drosophila (Teleman et al., 2005). Melted mutants are smaller than wild-type flies; however, in contrast to Drosophila mutants with reduced IIS (Bohni et al., 1999), they have reduced triglyceride stores. The Melted protein contains a C-terminal PH domain that mediates plasma membrane localization. Melted binds to dFoxO and promotes insulin-dependent localization of $\mathrm{dFoxO}$ to the plasma membrane. Accordingly, transcript levels of the $\mathrm{dFoxO}$ target gene 4E-BP are elevated in Melted mutants. Both the increase in 4E-BP expression as well as the reduction in total body triglycerides observed in Melted mutants are suppressed by mutations in $\mathrm{dFoxO}$. These observations suggest that Melted controls fat metabolism by regulating the expression of dFoxO target genes (Teleman et al., 2005). Although the C. elegans Melted ortholog K10B4.3 has not yet been implicated in IIS, RNAi of K10B4.3 extends C. elegans life span (Hamilton et al., 2005). Whether or not this phenotype requires daf-16/FoxO is not known.

A screen for modulators of Drosophila FoxO (dFoxO) activity has revealed the role of two conserved pathways in dFoxO regulation. RNAi of Drosophila ksr (kinase suppressor of Ras) enhances the dFoxO gain-of-function phenotype by reducing sensitivity to insulin, thus promoting dFoxO nuclear translocation (Zhang et al., 2011). $k s r$ was first identified as a protein that is required for Ras pathway action in C. elegans and Drosophila (Kornfeld et al., 1995; Sundaram and Han, 1995; Therrien et al., 1995). Accordingly, RNAi of Drosophila MEK also reduced sensitivity to insulin. Thus, the Drosophila Ras/Raf/MEK pathway antagonizes FoxO action (Zhang et al., 2011). Whether the C. elegans Ras pathway (Canonical RTK-Ras-ERK signaling and related alternative pathways) regulates DAF-16/FoxO activity is not known.

This same screen revealed a role for endoplasmic reticulum (ER) stress in dFoxO regulation. ER stress activates $\mathrm{dFoxO}$ by inducing $\mathrm{dFoxO}$ phosphorylation and subsequent nuclear translocation. $\mathrm{dFoxO}$ activation by ER stress requires phosphorylation of $\mathrm{dFoxO}$ at serine 243 by the ER-stress-dependent kinase PERK. PERK also phosphorylates human FoxO1 in vitro and potentiates FoxO1 activity in human cells, suggesting that FoxO regulation by ER stress is evolutionarily conserved (Zhang et al., 2013). A role for the C. elegans PERK ortholog PEK-1 in DAF-16/FoxO regulation and life span control has not yet been established, and the PERK phosphorylation site in dFoxO does not appear to be conserved in DAF-16/FoxO. However, ER stress likely influences DAF-16/FoxO activity, as the ER-stress-activated transcription factor XBP-1 is required for life span extension in $d a f-2 / I G F R$ mutants and collaborates with DAF-16/FoxO to regulate gene expression (Henis-Korenblit et al., 2010).

\section{Other transcription factors regulated by IIS}

Although genetic analysis indicates that DAF-16/FoxO is the major transcriptional output of IIS, other transcription factors that participate in the regulation of DAF-16/FoxO target gene expression are also regulated by IIS. For example, HSF-1, the heat shock transcription factor that controls stress-induced gene expression (Morley and Morimoto, 2004), extends life span in a DAF-16/FoxO-dependent manner when overexpressed (Hsu et al., 2003) and delays the onset of protein aggregation in animals expressing a fluorescent protein containing polyglutamine repeats (Morley et al., 2002; Hsu et al., 2003). HSF-1 and DAF-16/FoxO promote longevity by binding to HSEs (heat shock elements) and DBEs (DAF-16/FoxO binding elements) in the promoters of genes encoding small heat shock proteins (Hsu et al., 2003). Notably, HSF-1 is required for longevity induced by reduced 
IIS. Recent work has shown that IIS regulates HSF-1 activity by promoting the formation of a multimolecular complex containing the conserved proteins DDL-1 and DDL-2 that sequesters HSF-1 in the cytoplasm (Chiang et al., 2012a) (Figure 1).

The C. elegans Nrf family transcription factor SKN-1 is an important component of the organismal response to oxidative stress and is required for life span extension by dietary restriction or inhibition of the TORC1 or TORC2 pathways (An and Blackwell, 2003; Bishop and Guarente, 2007; Robida-Stubbs et al., 2012). SKN-1 regulates genes that defend against oxidative and xenobiotic stress, along with many genes involved in protein homeostasis and metabolism (An et al., 2005; Kahn et al., 2008; Oliveira et al., 2009; Li et al., 2011; Paek et al., 2012). Some of its downstream targets appear to be shared with DAF-16/FoxO (An et al., 2005; Oliveira et al., 2009). As is the case for HSF-1, SKN-1 also contributes to life span extension in animals with reduced IIS (Tullet et al., 2008). IIS inhibits SKN-1 activity by promoting its cytoplasmic sequestration through an RxRxxS/T motif that can be directly phosphorylated by Akt/PKB and related kinases (Tullet et al., 2008) (Figure 1).

The zinc finger transcription factor PQM-1 (Section 5.12) and DAF-16/FoxO are regulated reciprocally by IIS, as their nuclear localization appears to be mutually exclusive, and loss of daf-2/IGFR drives PQM-1 into the cytoplasm (Tepper et al., 2013).

\section{Components of IIS in other organisms that are conserved in C. elegans}

Other than C. elegans, insulin signaling has been most intensively studied in mammals and Drosophila. This section highlights evolutionarily conserved proteins and pathways that have been implicated in IIS in mammals and/or flies but do not have clear functions in C. elegans IIS.

\subsection{Ras signaling}

In mammalian cells, insulin and IGF-1 activate Ras signaling through the recruitment and phosphorylation of IRS and Shc adaptor proteins. Tyrosine phosphorylation of IRS proteins and Shc by ligand-activated insulin and IGF-1 receptors generates binding sites for the Grb2/Son-of-Sevenless (Sos) complex, thus promoting the translocation of the Sos guanine nucleotide exchange factor into proximity with membrane-associated Ras. Sos activates Ras by catalyzing the exchange of GDP bound to Ras for GTP. GTP-bound Ras activates the Raf/MEK/MAPK kinase cascade, leading to transcriptional regulatory events that promote mitogenesis (Siddle, 2011).

In C. elegans, the role of Ras in insulin signaling is less clear. C. elegans LET-60/Ras has a well-established role in vulval cell fate specification during larval development, where it is regulated by the epidermal growth factor receptor (EGFR) ortholog LET-23 (Canonical RTK-Ras-ERK signaling and alternative pathways). Based on the mammalian paradigm, DAF-2/IGFR activation would be predicted to activate LET-60/Ras. If activated LET-60/Ras transduces signals from DAF-2/IGFR, then reduction of LET-60/Ras signaling should promote dauer arrest and/or extend life span, whereas increases in LET-60/Ras activity should suppress the dauer-constitutive and life span extension phenotypes caused by reduced IIS. However, unlike age-1/PI3K, pdk-1, and akt-1 loss-of-function mutants, which have a dauer-constitutive phenotype, LET-60/Ras pathway loss-of-function mutations do not cause dauer arrest, suggesting that the PI3K/Akt pathway is the major output of DAF-2/IGFR. However, Ras pathway mutations do influence dauer arrest modestly in some (but not all) mutants with reduced IIS. For example, the let-60(n1046) gain-of-function (gf) mutation can suppress dauer arrest in daf-2(m577) mutants, and loss-of-function mutations in let-60/Ras and sem-5/Grb2 enhance daf-2(m577) mutant phenotypes (Nanji et al., 2005). These data suggest that Ras signaling has a weak modulatory effect on IIS in the control of dauer arrest. let-60( $g f)$ also partially suppresses the dauer-constitutive phenotype of the age-1(mg44) null mutant, indicating that Ras pathway action in dauer regulation is independent of PI3K (Nanji et al., 2005).

Ras pathway mutations have complex effects on life span extension in the context of reduced IIS. In contrast to the enhancement of the dauer-constitutive phenotype of daf-2/IGFR mutants by LET-60/Ras signaling loss of function, these same mutations partially suppress life span extension of daf-2(m577). Thus, Ras signaling has opposite effects on dauer-constitutive and life span extension phenotypes in the context of reduced IIS. Although let-60(gf) extends maximum life span in daf-2/IGFR mutants, it has no effect on mean life span due to increases in early adult mortality in daf-2; let-60(gf) double mutants (Nanji et al., 2005). The interpretation of these life span experiments is further complicated by the pleiotropies of Ras pathway mutants (Canonical RTK-Ras-ERK signaling and related alternative pathways). 
Whether Ras acts downstream of or parallel to DAF-2/IGFR to influence dauer arrest and life span remains uncertain: there is no biochemical data directly implicating the LET-60/Ras pathway as a target of $C$. elegans IIS, and there are differences in the functions of mammalian and C. elegans Shc (Neumann-Haefelin et al., 2008) and IRS proteins (Wolkow et al., 2002). It is also not known whether LET-60/Ras influences the activity of DAF-16/FoxO.

Although the Ras/MEK/MAPK pathway activated by insulin and IGF-1 in mammals appears to play a minor role in dauer regulation and life span control by IIS in C. elegans, DAF-2/IGFR does regulate this pathway in other contexts. For example, DAF-2/IGFR signaling potentiates Ras/MEK/MAPK signaling in vulval precursor cells during vulval development by promoting phosphorylation of the MAP kinase MPK-1 in an Akt- and DAF-16/FoxO-independent manner (Nakdimon et al., 2012), and DAF-2/IGFR signaling to LET-60/Ras may promote starvation-enhanced ribosome biogenesis in males (Tan et al., 2011). Conversely, in muscle, DAF-2/IGFR signals through AGE-1/PI3K and PDK-1 to inhibit Ras/MEK/MAPK-dependent protein degradation (Szewczyk et al., 2007).

\subsection{Shc}

The C. elegans genome encodes two Shc homologs, one of which (SHC-1) has been studied in some detail (Mizuno et al., 2008; Neumann-Haefelin et al., 2008). In contrast to mammalian Shc, which is a critical mediator of Ras pathway activation by insulin (Ravichandran, 2001), C. elegans SHC-1 does not appear to regulate Ras activity, as shc-1 mutants do not have phenotypes reminiscent of Ras pathway loss-of-function mutants (Mizuno et al., 2008). This may be a consequence of the fact that the major tyrosine phosphorylation sites on mammalian Shc that engage the GRB2/Sos complex are not conserved in SHC-1 (Luzi et al., 2000). This observation may also explain why Ras activation plays a more significant role in mammalian IIS than it appears to in C. elegans IIS. Although SHC-1 coprecipitates with DAF-2/IGFR when both are overexpressed in mammalian cell culture (Neumann-Haefelin et al., 2008), the biological significance of this interaction is not known.

SHC-1 has an established role in promoting stress resistance through a physical interaction with the MAP kinase kinase MEK-1 (Mizuno et al., 2008; Neumann-Haefelin et al., 2008). This interaction is required for resistance to heavy metals (Mizuno et al., 2008) and may also promote the nuclear translocation of DAF-16/FoxO in the context of environmental stress (Mizuno et al., 2008; Neumann-Haefelin et al., 2008).

\subsection{TOR}

The mammalian Target of Rapamycin (mTOR) is a conserved serine/threonine kinase that acts in distinct multiprotein complexes to control cell growth in response to nutrient availability and energy balance. mTOR

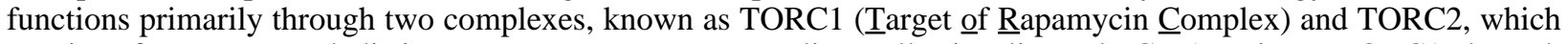
consist of common and distinct components. In mammalian cells, insulin and IGF-1 activate TORC1 through phosphorylation and inactivation of the TSC2 tumor suppressor by Akt (Avruch et al., 2006). By contrast, whether and how IIS couples to TOR in C. elegans is unclear. The long life span of let-363/TOR RNAi-treated animals is independent of daf-16/FoxO (Vellai et al., 2003). Instead, let-363/TOR appears to be part of the dietary restriction-mediated (DR) life span extension pathway (Hansen et al., 2007; Ching et al., 2010), which is genetically separable from IIS in C. elegans and requires the FoxA ortholog PHA-4 rather than DAF-16/FoxO (Panowski et al., 2007). The presence of nutrients promotes LET-363/TOR activity, which in turn represses PHA-4/FoxA activity, promoting development and normal life span (Sheaffer et al., 2008). Mutants of both let-363 and daf-15, the C. elegans ortholog of RAPTOR (regulatory associated protein of mTOR), form dauer-like larvae constitutively, and daf-15/RAPTOR heterozygotes are long-lived, like let-363/TOR knockdown animals (Jia et al., 2004). While there is no data to suggest that LET-363/TOR is regulated by IIS, daf-15/RAPTOR expression is negatively regulated by DAF-16/FoxO (Jia et al., 2004), providing a mechanism by which the IIS and DR pathways could be coordinated in growth vs. longevity decisions.

Although evidence is lacking for the regulation of TOR activity by IIS in C. elegans, TORC1 may regulate DAF-16/FoxO activity independently of IIS. The effect of TORC1 activation on DAF-16/FoxO appears to be context-dependent. The $C$. elegans ortholog of the TORC1 activator Rheb, RHEB-1, is required for life span extension in response to intermittent fasting (IF) (Honjoh et al., 2009). IF induces DAF-16/FoxO nuclear translocation and DAF-16/FoxO target gene expression, both of which require RHEB-1 and LET-363/TOR activity (Honjoh et al., 2009). By contrast, under ad lib feeding conditions, TORC1 inhibition extends life span in a DAF-16/FoxO-dependent manner and promotes DAF-16/FoxO target gene expression and nuclear translocation of 
the D/F/H isoform of DAF-16/FoxO (Robida-Stubbs et al., 2012). Thus, TORC1 promotes DAF-16/FoxO activity in the context of IF but inhibits DAF-16/FoxO activity in well-fed animals. The molecular basis for DAF-16/FoxO regulation by TORC1 has not been established.

\subsection{Glucose transport}

In mammals, insulin regulates glucose uptake in muscle and adipose tissue by promoting the translocation of the facilitative glucose transporter GLUT4 to the plasma membrane. This process requires the activation of both canonical IRS/PI3K/Akt signaling as well as a PI3K-independent pathway involving the tyrosine phosphorylation of the adaptor proteins APS and $\mathrm{Cbl}$ and the subsequent nucleation of a protein complex that activates the small GTPase TC10 in plasma membrane lipid rafts (Chang et al., 2004). Although the C. elegans genome encodes members of the glucose transporter superfamily, there is no evidence to suggest that glucose transport is regulated by ILPs or DAF-2/IGFR. This may be due in part to the fact that, although the PI3K/Akt pathway and Cbl are conserved in C. elegans, the $C$. elegans genome appears to lack an ortholog of APS, the tyrosine phosphorylation of which facilitates insulin-dependent recruitment of $\mathrm{Cbl}$ to the insulin receptor and insulin-dependent translocation of GLUT4 to the plasma membrane (Liu et al., 2002). However, glucose does shorten $C$. elegans life span in an IIS-dependent manner, and ins-7 expression increases upon glucose exposure, suggesting that $C$. elegans IIS does respond to changes in glucose levels (Lee et al., 2009).

\subsection{Cytohesins}

The Drosophila cytohesin ortholog Steppke was identified as a gene required for larval growth (Fuss et al., 2006). It acts in the PI3K/Akt pathway to control dFoxO target gene expression. The triazole derivative SecinH3, which inhibits the guanine nucleotide exchange activity of cytohesins, reduces insulin-induced Akt phosphorylation and blocks insulin-dependent regulation of 4E-BP and insulin receptor transcript abundance in Drosophila S2 cells; accordingly, SecinH3 inhibits insulin signaling in human HepG2 cells and causes hepatic insulin resistance in mice (Fuss et al., 2006; Hafner et al., 2006). Thus, cytohesins have a conserved role in promoting insulin/PI3K/Akt signaling. Although the C. elegans genome contains a cytohesin ortholog (K06H7.4), a potential role in IIS has not been reported.

\section{System-wide integration of IIS: non-autonomy and interaction with other pathways}

Dauer entry, longevity, and reproduction in any individual are determined by the integration of information about nutrient availability, number of neighbors (or competitors), and temperature; these variables may be interpreted as predictors of future survival and reproductive conditions. Much of the C. elegans systemic response to these environmental factors is mediated through IIS and its interactions with other signaling pathways.

\subsection{IIS-mediated cell non-autonomous regulation of longevity, dauer formation, metabolism, and reproduction:}

Larval and adult expression of a daf-2p::GFP promoter fusion has been reported in the intestine and nervous system (Hunt-Newbury et al., 2007), and recent immunofluorescence studies with affinity-purified anti-DAF-2 antibodies suggest that DAF-2/IGFR is most highly expressed in neurons and in the XXX secretory cells (which produce dafachronic acid and regulate dauer formation (Schaedel et al., 2012)), with weaker fluorescence in non-neuronal tissues, such as hypodermis (Kimura et al., 2011). Using mosaic analysis (Nomarski images for learning the anatomy, with tips for mosaic analysis), Apfeld and Kenyon found that DAF-2/IGFR acts cell non-autonomously to regulate dauer formation, longevity, and intestinal pigmentation associated with fat accumulation (Apfeld and Kenyon, 1998). To identify the site of IIS regulation of longevity and metabolism, Wolkow et al. (2000) used tissue-specific promoters to rescue daf-2/IGFR in specific tissues in daf-2(-) mutants. They found that daf-2/IGFR expression in neurons and intestine, but not in muscle, rescued longevity and dauer phenotypes, while restoration in muscle rescued metabolic defects (Wolkow et al., 2000). These results suggest that DAF-2/IGFR acts primarily in the neurons to control such system-wide phenotypes as longevity and dauer formation, but may have cell autonomous metabolic functions, at least when expressed at high levels in the intestine. The reason for the apparent discrepancy between the Apfeld and Kenyon and Wolkow et al. results might be due to technical differences (such as variation and non-endogenous levels of gene expression), but together the results do suggest that IIS in neurons is critical for non-autonomous regulation of longevity and dauer formation (Apfeld and Kenyon, 1998; Wolkow et al., 2000). Furthermore, the results imply that a downstream, secondary signal likely communicates with other tissues to coordinate the dauer decision and longevity response. 
The tissue-specific and cell autonomous roles of DAF-16/FoxO have also been investigated. The daf-16a and $d a f-16 d / f / h$ isoforms are expressed in most tissues, whereas the $d a f-16 b$ isoform has a more limited tissue distribution (Section 5.1) (Lin et al., 1997; Ogg et al., 1997; Lee et al., 2001; Kwon et al., 2010). Similar to the daf-2/IGFR mosaic and neuronal rescue results (Apfeld and Kenyon, 1998; Wolkow et al., 2000), expression of DAF-16A in the nervous system restored dauer formation in a significant fraction of daf-16; daf-2 double mutant animals (Libina et al., 2003), suggesting the existence of an organism-wide response to DAF-16/FoxO activation in neurons. Intriguingly, these animals arrest as dauers in the absence of DAF-16/FoxO activity in other important tissues such as the intestine and hypodermis. When DAF-16A is expressed solely in the intestine in a daf-16; daf-2 double mutant background, life span is increased up to $60 \%$, while its expression in other tissues, including neurons, had little to no effect on longevity (Libina et al., 2003). These results imply that DAF-16/FoxO may have tissue-specific longevity targets, or that the intestine is particularly sensitive to age-related damage (Libina et al., 2003). Currently, there is no data on the role of DAF-16/FoxO in the hypodermis, a tissue that is increasingly recognized for its role in endocrine function.

In addition to its longevity phenotype, daf-16/FoxO restoration in the intestine also rescued reproductive aging, indicating a novel role for DAF-16/FoxO in this tissue (Luo et al., 2010). Surprisingly, DAF-16/FoxO expression in the muscle also rescued reproductive aging, suggesting that the muscle plays a previously unappreciated role in IIS/DAF-16 regulation of reproduction (Luo et al., 2010).

Signaling between tissues, employing both insulin-like peptides (Murphy et al., 2007) and lipophilic hormones (Gerisch et al., 2001; Jia et al., 2002; Mak and Ruvkun, 2004), likely coordinates IIS activity and longevity state throughout the organism. For example, DAF-16/FoxO activity in one tissue can regulate its activity in other tissues, suggesting that DAF-16/FoxO regulates IIS activity in neighboring cells through downstream signaling (Libina et al., 2003). This feed-forward activation of DAF-16/FoxO (FoxO-to-FoxO signaling) in the regulation of longevity is mediated largely by the activity of the INS-7 ILP (Murphy et al., 2007). DAF-16/FoxO activity in the intestine and muscle likely activate signaling to the germline, although the nature of this signal (or signals) and its receptor is unknown. Reproductive aging is also regulated by IIS in the intestine and muscles, but the signal for this non-autonomous activity is unknown (Luo et al., 2010).

\subsection{IIS interactions with the dafachronic acid/DAF-12 pathway}

Genetic analysis underscores the complexity of interactions between IIS and the dafachronic acid/DAF-12 pathway (for more details on this pathway see WormBook chapter Dauer). Mutants that lack dafachronic acid (in which the DAF-12 nuclear receptor is unliganded) undergo dauer arrest independently of DAF-16/FoxO (Gerisch et al., 2001; Jia et al., 2002), suggesting that unliganded DAF-12 acts downstream of IIS. However, studies with eak mutants have shown that the expression of some DAF-16/FoxO target genes requires DAF-12 activity (see Section 6.2), indicating that DAF-16/FoxO and DAF-12 may act in parallel in some contexts (Zhang et al., 2008; Alam et al., 2010; Dumas et al., 2010). Furthermore, whereas daf-12 null mutations fully suppress dauer arrest in most dauer-constitutive mutant backgrounds, including weaker daf-2/IGFR loss-of-function alleles such as e1368, daf-2(e1370); daf-12(null) double mutants have a synthetic phenotype, undergoing non-dauer larval arrest at $25^{\circ} \mathrm{C}$ (Dumas et al., 2013b). Recent work on the interactions of IIS and DA/DAF-12 pathways in life span control suggest that, whereas liganded DAF-12 promotes longevity in the context of reduced IIS, unliganded DAF-12 can either shorten or prolong life span in animals with reduced IIS (Dumas et al., 2013b).

\subsection{IIS/TGF- $\beta$ crosstalk}

The TGF- $\beta$ pathway that controls dauer arrest (see WormBook chapter Dauer) also regulates the expression of ins-7 and ins-18 (Liu et al., 2004; Shaw et al., 2007), linking TGF- $\beta$ dauer regulatory activity to IIS, possibly in order to coordinate dauer and longevity decisions through multiple signaling inputs (Shaw et al., 2007). A recent study of the pyruvate dehydrogenase phosphatase homolog PDP-1, which acts in the TGF- $\beta$ dauer pathway to affect insulin signaling upstream of IIS (Narasimhan et al., 2011), supports such a model of IIS/TGF- $\beta$ signaling coordination through ILP regulation (Shaw et al., 2007).

\subsection{IIS coordination of reproduction and longevity}

Communication from the soma to the germline, and from the germline back to the soma (Hsin and Kenyon, 1999; Luo et al., 2010), might enable an organism to tune its reproductive span and life span properly to adapt to changing nutrient availability (Luo and Murphy, 2011). While the signals between the tissues are unknown, IIS plays a key role in each arm of this coordinating program, highlighting its importance in the regulation of important life history traits. 


\section{Conclusions and Future Directions}

Here we have discussed the major components of C. elegans IIS and its impact on many phenotypes and behaviors, including development, longevity, stress resistance, and reproduction. Despite the vast amount of information that study of this pathway in C. elegans has revealed, important questions remain. For example, IIS has both cell autonomous and non-autonomous functions, and the activity of IIS in each tissue is not yet known. Tissue specificity has only been addressed in a few studies, and there is a disconnect between the $d a f-2 / I G F R$ and daf-16/FoxO results regarding their roles in particular tissues. Furthermore, most genome-wide experiments (e.g., microarrays, SAGE, ChIP-Seq) performed on whole animals have not revealed insights into tissue-specific targets of IIS and DAF-16/FoxO. In fact, many of the organismal phenotypes studied in C. elegans IIS mutants (longevity, dauer formation, pathogen survival, stress resistance) are the result of system-wide signal integration. For most of these phenotypes, the communicating signals between tissues are still unknown.

IIS influences important life history phenotypes and integrates signaling information at least in part through interactions with other signaling pathways (including TGF- $\beta$, dafachronic acid/DAF-12, and Ras/Raf/MAP kinase pathways) and important transcription factors. Nonetheless, it is likely that the extent of these interactions is not yet fully appreciated.

ILPs may play a role in tissue-specific responses and coordination, but the sheer number of ILPs in C. elegans raises another set of questions. Where do they all act? What do they all do? Why are there so many? Do they all act through DAF-2/IGFR, or are there alternative receptors for ILPs?

What is the cost of the increased robustness of IIS pathway mutants (said another way, why are all worms not IIS mutants?)? First and foremost, IIS mutants are slow to develop, they produce slightly fewer progeny (particularly early progeny), and they produce them over a longer period of time; together, these delays result in lower fitness when compared to wild-type worms, despite the fact that IIS mutants are more stress resistant (Walker et al., 2000; Jenkins et al., 2004). However, IIS mutants are better able to cope with stressful conditions in almost all stages of life. For example, under stressful conditions in early development, IIS controls the trigger for dauer formation, and starvation-induced L1 arrest is also IIS-dependent. With milder stresses, the same pathway can respond with protective transcriptional responses that extend somatic longevity, germline proliferation, and oocyte quality maintenance. Together, these data suggest that the responsiveness of the insulin/IGF-1-like signaling pathway may allow $C$. elegans to quickly respond to stressful conditions, allowing them to live to fight, or at least to reproduce and survive, another day.

\section{Acknowledgments}

The authors thank members of the Hu and Murphy labs for critical evaluation of the manuscript. CTM is the Richard B. Fisher Preceptor in Integrative Genomics at Princeton University.

\section{References}

Accili, D., and Arden, K.C. (2004). FoxOs at the crossroads of cellular metabolism, differentiation, and transformation. Cell 117, 421-426. Abstract Article

Ailion, M., and Thomas, J.H. (2003). Isolation and characterization of high-temperature-induced Dauer formation mutants in Caenorhabditis elegans. Genetics 165, 127-144. Abstract

Alam, H., Williams, T.W., Dumas, K.J., Guo, C., Yoshina, S., Mitani, S., and Hu, P.J. (2010). EAK-7 controls development and life span by regulating nuclear DAF-16/FoxO activity. Cell Metab. 12, 30-41. Abstract

An, J.H., and Blackwell, T.K. (2003). SKN-1 links C. elegans mesendodermal specification to a conserved oxidative stress response. Genes Dev. 17, 1882-1893. Abstract Article

An, J.H., Vranas, K., Lucke, M., Inoue, H., Hisamoto, N., Matsumoto, K., and Blackwell, T.K. (2005). Regulation of the Caenorhabditis elegans oxidative stress defense protein SKN-1 by glycogen synthase kinase-3. Proc. Natl. Acad. Sci. U. S. A. 102, 16275-16280. Abstract Article 
Ansley, S.J., Badano, J.L., Blacque, O.E., Hill, J., Hoskins, B.E., Leitch, C.C., Kim, J.C., Ross, A.J., Eichers, E.R., Teslovich, T.M., et al. (2003). Basal body dysfunction is a likely cause of pleiotropic Bardet-Biedl syndrome. Nature 425, 628-633. Abstract Article

Antebi, A., Yeh, W.H., Tait, D., Hedgecock, E.M., and Riddle, D.L. (2000). daf-12 encodes a nuclear receptor that regulates the dauer diapause and developmental age in C. elegans. Genes Dev. 14, 1512-1527. Abstract Article

Apfeld, J., and Kenyon, C. (1998). Cell nonautonomy of C. elegans daf-2 function in the regulation of diapause and life span. Cell 95, 199-210. Abstract Article

Apfeld, J., O'Connor, G., McDonagh, T., DiStefano, P.S., and Curtis, R. (2004). The AMP-activated protein kinase AAK-2 links energy levels and insulin-like signals to lifespan in C. elegans. Genes Dev. 18, 3004-3009. Abstract Article

Arantes-Oliveira, N., Berman, J.R., and Kenyon, C. (2003). Healthy animals with extreme longevity. Science 302, 611. Abstract Article

Arasu, P., Wightman, B., and Ruvkun, G. (1991). Temporal regulation of lin-14 by the antagonistic action of two other heterochronic genes, lin-4 and lin-28. Genes Dev. 5, 1825-1833. Abstract

Arkblad, E.L., Tuck, S., Pestov, N.B., Dmitriev, R.I., Kostina, M.B., Stenvall, J., Tranberg, M., and Rydstrom, J. (2005). A Caenorhabditis elegans mutant lacking functional nicotinamide nucleotide transhydrogenase displays increased sensitivity to oxidative stress. Free Radic. Biol. Med. 38, 1518-1525. Abstract Article

Ashrafi, K., Chang, F.Y., Watts, J.L., Fraser, A.G., Kamath, R.S., Ahringer, J., and Ruvkun, G. (2003). Genome-wide RNAi analysis of Caenorhabditis elegans fat regulatory genes. Nature 421, 268-272. Abstract Article

Avruch, J., Hara, K., Lin, Y., Liu, M., Long, X., Ortiz-Vega, S., and Yonezawa, K. (2006). Insulin and amino-acid regulation of mTOR signaling and kinase activity through the Rheb GTPase. Oncogene 25, 6361-6372. Abstract Article

Ayyadevara, S., Alla, R., Thaden, J.J., and Shmookler Reis, R.J. (2008). Remarkable longevity and stress resistance of nematode PI3K-null mutants. Aging Cell 7, 13-22. Abstract Article

Babar, P., Adamson, C., Walker, G.A., Walker, D.W., and Lithgow, G.J. (1999). P13-kinase inhibition induces dauer formation, thermotolerance and longevity in C. elegans. Neurobiol. Aging 20,513-519. Abstract

Barsyte, D., Lovejoy, D.A., and Lithgow, G.J. (2001). Longevity and heavy metal resistance in daf-2 and age-1 long-lived mutants of Caenorhabditis elegans. FASEB J. 15, 627-634. Abstract Article

Baugh, L.R., and Sternberg, P.W. (2006). DAF-16/FOXO regulates transcription of $c k i-1 /$ Cip/Kip and repression of lin-4 during C. elegans L1 arrest. Curr. Biol. 16, 780-785. Abstract Article

Berdichevsky, A., Viswanathan, M., Horvitz, H.R., and Guarente, L. (2006). C. elegans SIR-2.1 interacts with 14-3-3 proteins to activate DAF-16 and extend life span. Cell 125, 1165-1177. Abstract Article

Berman, J.R., and Kenyon, C. (2006). Germ-cell loss extends C. elegans life span through regulation of DAF-16 by kri-l and lipophilic-hormone signaling. Cell 124, 1055-1068. Abstract Article

Billing, O., Kao, G., and Naredi, P. (2011). Mitochondrial function is required for secretion of DAF-28/insulin in $C$. elegans. PLoS One 6, e14507. Abstract Article

Bishop, N.A., Guarente, L. (2007). Two neurons mediate diet-restriction-induced longevity in C. elegans. Nature 447, 545-549. Abstract Article

Bluher, M., Kahn, B.B., and Kahn, C.R. (2003). Extended longevity in mice lacking the insulin receptor in adipose tissue. Science 299, 572-574. Abstract Article 
Bodenmiller, B., Campbell, D., Gerrits, B., Lam, H., Jovanovic, M., Picotti, P., Schlapbach, R., and Aebersold, R. (2008). PhosphoPep - a database of protein phosphorylation sites in model organisms. Nat. Biotech. 26, 1339-1340. Abstract Article

Bohni, R., Riesgo-Escovar, J., Oldham, S., Brogiolo, W., Stocker, H., Andruss, B.F., Beckingham, K., and Hafen, E. (1999). Autonomous control of cell and organ size by CHICO, a Drosophila homolog of vertebrate IRS1-4. Cell 97, 865-875. Abstract Article

Boulias, K., and Horvitz, H.R. (2012). The C. elegans microRNA mir-71 acts in neurons to promote germline-mediated longevity through regulation of DAF-16/FOXO. Cell Metab. 15, 439-450. Abstract Article

Bozulic, L., and Hemmings, B.A. (2009). PIKKing on PKB: regulation of PKB activity by phosphorylation. Curr. Opin. Cell Biol. 21, 256-261. Abstract Article

Brent, M.M., Anand, R., and Marmorstein, R. (2008). Structural basis for DNA recognition by FoxO1 and its regulation by posttranslational modification. Structure 16, 1407-1416. Abstract Article

Bruhn, M.A., Pearson, R.B., Hannan, R.D., and Sheppard, K.E. (2010). Second AKT: The rise of SGK in cancer signalling. Growth Factors 28, 394-408. Abstract Article

Brunet, A., Bonni, A., Zigmond, M.J., Lin, M.Z., Juo, P., Hu, L.S., Anderson, M.J., Arden, K.C., Blenis, J., and Greenberg, M.E. (1999). Akt promotes cell survival by phosphorylating and inhibiting a Forkhead transcription factor. Cell 96, 857-868. Abstract Article

Brunet, A., Park, J., Tran, H., Hu, L.S., Hemmings, B.A., and Greenberg, M.E. (2001). Protein kinase SGK mediates survival signals by phosphorylating the forkhead transcription factor FKHRL1 (FOXO3a). Mol. Cell. Biol. 21, 952-965. Abstract Article

Brunet, A., Sweeney, L.B., Sturgill, J.F., Chua, K.F., Greer, P.L., Lin, Y., Tran, H., Ross, S.E., Mostoslavsky, R., Cohen, H.Y., et al. (2004). Stress-dependent regulation of FOXO transcription factors by the SIRT1 deacetylase. Science 303, 2011-2015. Abstract Article

Burnett, C., Valentini, S., Cabreiro, F., Goss, M., Somogyvari, M., Piper, M.D., Hoddinott, M., Sutphin, G.L., Leko, V., McElwee, J.J., et al. (2011). Absence of effects of Sir2 overexpression on lifespan in C. elegans and Drosophila. Nature 477, 482-485. Abstract Article

Cantrell, D.A. (2001). Phosphoinositide 3-kinase signalling pathways. J. Cell Sci. 114, 1439-1445. Abstract

Chalasani, S.H., Kato, S., Albrecht, D.R., Nakagawa, T., Abbott, L.F., and Bargmann, C.I. (2010). Neuropeptide feedback modifies odor-evoked dynamics in Caenorhabditis elegans olfactory neurons. Nat. Neurosci. 13, 615-621. Abstract Article

Chang, L., Chiang, S.H., and Saltiel, A.R. (2004). Insulin signaling and the regulation of glucose transport. Mol. Med. 10, 65-71. Abstract Article

Chen, A.T.-Y., Guo, C., Dumas, K.J., Ashrafi, K., and Hu, P.J. (2013a). Effects of C. elegans sgk-1 mutations on life span, stress resistance, and DAF-16/FoxO regulation. Aging Cell 12, 932-940. Abstract Article

Chen, Z., Hendricks, M., Cornils, A., Maier, W., Alcedo, J., and Zhang, Y. (2013b). Two insulin-like peptides antagonistically regulate aversive olfactory learning in C. elegans. Neuron 77, 572-585. Abstract Article

Chiang, W.C., Ching, T.T., Lee, H.C., Mousigian, C., and Hsu, A.L. (2012a). HSF-1 regulators DDL-1/2 link insulin-like signaling to heat-shock responses and modulation of longevity. Cell 148, 322-334. Abstract Article

Chiang, W.C., Tishkoff, D.X., Yang, B., Wilson-Grady, J., Yu, X., Mazer, T., Eckersdorff, M., Gygi, S.P., Lombard, D.B., and Hsu, A.L. (2012b). C. elegans SIRT6/7 homolog SIR-2.4 promotes DAF-16 relocalization and function during stress. PLoS Genet. 8, e1002948. Abstract Article 
Ching, T.T., Chiang, W.C., Chen, C.S., and Hsu, A.L. (2011). Celecoxib extends C. elegans lifespan via inhibition of insulin-like signaling but not cyclooxygenase-2 activity. Aging Cell 10, 506-519. Abstract Article

Ching, T.T., Paal, A.B., Mehta, A., Zhong, L., and Hsu, A.L. (2010). $d r r-2$ encodes an eIF4H that acts downstream of TOR in diet-restriction-induced longevity of $C$. elegans. Aging Cell 9, 545-557. Abstract Article

Christensen, R., de la Torre-Ubieta, L., Bonni, A., and Colon-Ramos, D.A. (2011). A conserved PTEN/FOXO pathway regulates neuronal morphology during C. elegans development. Development 138, 5257-5267. Abstract Article

Clancy, D.J., Gems, D., Harshman, L.G., Oldham, S., Stocker, H., Hafen, E., Leevers, S.J., and Partridge, L. (2001). Extension of life-span by loss of CHICO, a Drosophila insulin receptor substrate protein. Science 292, 104-106. Abstract

Cohen, E., Bieschke, J., Perciavalle, R.M., Kelly, J.W., and Dillin, A. (2006). Opposing activities protect against age-onset proteotoxicity. Science 313, 1604-1610. Abstract Article

Cornils, A., Gloeck, M., Chen, Z., Zhang, Y., and Alcedo, J. (2011). Specific insulin-like peptides encode sensory information to regulate distinct developmental processes. Development 138, 1183-1193. Abstract Article

Daitoku, H., Hatta, M., Matsuzaki, H., Aratani, S., Ohshima, T., Miyagishi, M., Nakajima, T., and Fukamizu, A. (2004). Silent information regulator 2 potentiates Foxo1-mediated transcription through its deacetylase activity. Proc. Natl. Acad. Sci. U. S. A. 101, 10042-10047. Abstract Article

Datta, S.R., Brunet, A., and Greenberg, M.E. (1999). Cellular survival: a play in three Akts. Genes Dev. 13, 2905-2927. Abstract

David, D.C., Ollikainen, N., Trinidad, J.C., Cary, M.P., Burlingame, A.L., and Kenyon, C. (2010). Widespread protein aggregation as an inherent part of aging in C. elegans. PLoS Biol. 8, e1000450. Abstract Article

de Lencastre, A., Pincus, Z., Zhou, K., Kato, M., Lee, S.S., and Slack, F.J. (2010). MicroRNAs both promote and antagonize longevity in C. elegans. Curr. Biol. 20, 2159-2168. Abstract Article

Dillin, A., Crawford, D.K., and Kenyon, C. (2002). Timing requirements for insulin/IGF-1 signaling in C. elegans. Science 298, 830-834. Abstract Article

Dlakic, M. (2002). A new family of putative insulin receptor-like proteins in C. elegans. Curr. Biol. 12, R155-157. Abstract

Dong, M.Q., Venable, J.D., Au, N., Xu, T., Park, S.K., Cociorva, D., Johnson, J.R., Dillin, A., and Yates, J.R., 3rd (2007). Quantitative mass spectrometry identifies insulin signaling targets in C. elegans. Science 317, 660-663. Abstract Article

Dorman, J.B., Albinder, B., Shroyer, T., and Kenyon, C. (1995). The age-1 and daf-2 genes function in a common pathway to control the lifespan of Caenorhabditis elegans. Genetics 141, 1399-1406. Abstract

Downes, C.P., Perera, N., Ross, S., and Leslie, N.R. (2007). Substrate specificity and acute regulation of the tumour suppressor phosphatase, PTEN. Biochem. Soc. Symp. 74, 69-80. Abstract

Duhon, S.A., and Johnson, T.E. (1995). Movement as an index of vitality: comparing wild type and the age-1 mutant of Caenorhabditis elegans. J. Gerontol. A Biol. Sci. Med. Sci. 50, B254-261. Abstract Article

Dumas, K.J., Delaney, C.E., Flibotte, S., Moerman, D.G., Csankovszki, G., and Hu, P.J. (2013a). Unexpected role for dosage compensation in the control of dauer arrest, insulin-like signaling, and FoxO transcription factor activity in Caenorhabditis elegans. Genetics 194, 619-629. Abstract Article

Dumas, K.J., Guo, C., Shih, H.J., and Hu, P.J. (2013b). Influence of steroid hormone signaling on life span control by Caenorhabditis elegans insulin-like signaling. G3 3, 841-850. Abstract Article 
Dumas, K.J., Guo, C., Wang, X., Burkhart, K.B., Adams, E.J., Alam, H., and Hu, P.J. (2010). Functional divergence of dafachronic acid pathways in the control of C. elegans development and lifespan. Dev. Biol. 340, 605-612. Abstract Article

Duret, L., Guex, N., Peitsch, M.C., and Bairoch, A. (1998). New insulin-like proteins with atypical disulfide bond pattern characterized in Caenorhabditis elegans by comparative sequence analysis and homology modeling. Genome Res. 8, 348-353. Abstract Article

Essers, M.A., de Vries-Smits, L.M., Barker, N., Polderman, P.E., Burgering, B.M., and Korswagen, H.C. (2005). Functional interaction between $\beta$-catenin and FOXO in oxidative stress signaling. Science 308, 1181-1184. Abstract Article

Essers, M.A., Weijzen, S., de Vries-Smits, A.M., Saarloos, I., de Ruiter, N.D., Bos, J.L., and Burgering, B.M. (2004). FOXO transcription factor activation by oxidative stress mediated by the small GTPase Ral and JNK. EMBO J. 23, 4802-4812. Abstract Article

Evans, E.A., Kawli, T., and Tan, M.W. (2008). Pseudomonas aeruginosa suppresses host immunity by activating the DAF-2 insulin-like signaling pathway in Caenorhabditis elegans. PLoS Pathog. 4, e1000175. Abstract Article

Felix, M.A., Ashe, A., Piffaretti, J., Wu, G., Nuez, I., Belicard, T., Jiang, Y., Zhao, G., Franz, C.J., Goldstein, L.D., et al. (2011). Natural and experimental infection of Caenorhabditis nematodes by novel viruses related to nodaviruses. PLoS Biol. 9, e1000586. Abstract Article

Flatt, T., Min, K.J., D'Alterio, C., Villa-Cuesta, E., Cumbers, J., Lehmann, R., Jones, D.L., and Tatar, M. (2008). Drosophila germ-line modulation of insulin signaling and lifespan. Proc. Natl. Acad. Sci. U. S. A. 105, 6368-6373. Abstract Article

Francis, R., Barton, M.K., Kimble, J., and Schedl, T. (1995). gld-1, a tumor suppressor gene required for oocyte development in Caenorhabditis elegans. Genetics 139, 579-606. Abstract

Friedman, D.B., and Johnson, T.E. (1988a). A mutation in the age-1 gene in Caenorhabditis elegans lengthens life and reduces hermaphrodite fertility. Genetics 118, 75-86. Abstract Article

Friedman, D.B., and Johnson, T.E. (1988b). Three mutants that extend both mean and maximum life span of the nematode, Caenorhabditis elegans, define the age-1 gene. J. Gerontol. 43, B102-109. Abstract Article

Fu, W., Ma, Q., Chen, L., Li, P., Zhang, M., Ramamoorthy, S., Nawaz, Z., Shimojima, T., Wang, H., Yang, Y., et al. (2009). MDM2 acts downstream of p53 as an E3 ligase to promote FOXO ubiquitination and degradation. J. Biol. Chem. 284, 13987-14000. Abstract Article

Fukuyama, M., Rougvie, A.E., and Rothman, J.H. (2006). C. elegans DAF-18/PTEN mediates nutrient-dependent arrest of cell cycle and growth in the germline. Curr. Biol. 16, 773-779. Abstract Article

Furnari, F.B., Huang, H.J., and Cavenee, W.K. (1998). The phosphoinositol phosphatase activity of PTEN mediates a serum-sensitive G1 growth arrest in glioma cells. Cancer Res. 58, 5002-5008. Abstract

Furuyama, T., Nakazawa, T., Nakano, I., and Mori, N. (2000). Identification of the differential distribution patterns of mRNAs and consensus binding sequences for mouse DAF-16 homologues. Biochem. J. 349, 629-634. Abstract

Fuss, B., Becker, T., Zinke, I., and Hoch, M. (2006). The cytohesin Steppke is essential for insulin signalling in Drosophila. Nature 444, 945-948. Abstract Article

Garigan, D., Hsu, A.L., Fraser, A.G., Kamath, R.S., Ahringer, J., and Kenyon, C. (2002). Genetic analysis of tissue aging in Caenorhabditis elegans. A role for heat-shock factor and bacterial proliferation. Genetics 161, 1101-1112. Abstract

Garsin, D.A., Villanueva, J.M., Begun, J., Kim, D.H., Sifri, C.D., Calderwood, S.B., Ruvkun, G., and Ausubel, F.M. (2003). Long-lived C. elegans daf-2 mutants are resistant to bacterial pathogens. Science 300, 1921. Abstract Article 
Gems, D., Sutton, A.J., Sundermeyer, M.L., Albert, P.S., King, K.V., Edgley, M.L., Larsen, P.L., and Riddle, D.L. (1998). Two pleiotropic classes of daf-2 mutation affect larval arrest, adult behavior, reproduction and longevity in Caenorhabditis elegans. Genetics 150, 129-155. Abstract

Gerisch, B., Rottiers, V., Li, D., Motola, D.L., Cummins, C.L., Lehrach, H., Mangelsdorf, D.J., and Antebi, A. (2007). A bile acid-like steroid modulates Caenorhabditis elegans lifespan through nuclear receptor signaling. Proc. Natl. Acad. Sci. U. S. A. 104, 5014-5019. Abstract Article

Gerisch, B., Weitzel, C., Kober-Eisermann, C., Rottiers, V., and Antebi, A. (2001). A hormonal signaling pathway influencing C. elegans metabolism, reproductive development, and life span. Dev. Cell 1, 841-851. Abstract Article

Ghazi, A., Henis-Korenblit, S., and Kenyon, C. (2007). Regulation of Caenorhabditis elegans lifespan by a proteasomal E3 ligase complex. Proc. Natl. Acad. Sci. U. S. A. 104, 5947-5952. Abstract Article

Ghazi, A., Henis-Korenblit, S., and Kenyon, C. (2009). A transcription elongation factor that links signals from the reproductive system to lifespan extension in Caenorhabditis elegans. PLoS Genet. 5, e1000639. Abstract Article

Gil, E.B., Malone Link, E., Liu, L.X., Johnson, C.D., and Lees, J.A. (1999). Regulation of the insulin-like developmental pathway of Caenorhabditis elegans by a homolog of the PTEN tumor suppressor gene. Proc. Natl. Acad. Sci. U. S. A. 96, 2925-2930. Abstract Article

Gottlieb, S., and Ruvkun, G. (1994). daf-12, daf-16 and daf-23: genetically interacting genes controlling Dauer formation in Caenorhabditis elegans. Genetics 137, 107-120. Abstract

Goudeau, J., Bellemin, S., Toselli-Mollereau, E., Shamalnasab, M., Chen, Y., and Aguilaniu, H. (2011). Fatty acid desaturation links germ cell loss to longevity through NHR-80/HNF4 in C. elegans. PLoS Biol. 9, e1000599. Abstract Article

Greer, E.L., Dowlatshahi, D., Banko, M.R., Villen, J., Hoang, K., Blanchard, D., Gygi, S.P., and Brunet, A. (2007a). An AMPK-FOXO pathway mediates longevity induced by a novel method of dietary restriction in C. elegans. Curr. Biol. 17, 1646-1656. Abstract Article

Greer, E.L., Oskoui, P.R., Banko, M.R., Maniar, J.M., Gygi, M.P., Gygi, S.P., and Brunet, A. (2007b). The energy sensor AMP-activated protein kinase directly regulates the mammalian FOXO3 transcription factor. J. Biol. Chem. 282, 30107-30119. Abstract Article

Hafner, M., Schmitz, A., Grune, I., Srivatsan, S.G., Paul, B., Kolanus, W., Quast, T., Kremmer, E., Bauer, I., and Famulok, M. (2006). Inhibition of cytohesins by SecinH3 leads to hepatic insulin resistance. Nature 444, 941-944. Abstract Article

Halaschek-Wiener, J., Khattra, J.S., McKay, S., Pouzyrev, A., Stott, J.M., Yang, G.S., Holt, R.A., Jones, S.J., Marra, M.A., Brooks-Wilson, A.R., et al. (2005). Analysis of long-lived C. elegans daf-2 mutants using serial analysis of gene expression. Genome Res. 15, 603-615. Abstract

Hamilton, B., Dong, Y., Shindo, M., Liu, W., Odell, I., Ruvkun, G., and Lee, S.S. (2005). A systematic RNAi screen for longevity genes in C. elegans. Genes Dev. 19, 1544-1555. Abstract Article

Hansen, M., Taubert, S., Crawford, D., Libina, N., Lee, S.J., and Kenyon, C. (2007). Lifespan extension by conditions that inhibit translation in Caenorhabditis elegans. Aging Cell 6, 95-110. Abstract Article

Hardie, D.G., Ross, F.A., and Hawley, S.A. (2012). AMPK: a nutrient and energy sensor that maintains energy homeostasis. Nat. Rev. Mol. Cell Biol. 13, 251-262. Abstract Article

Hellerer, T., Axang, C., Brackmann, C., Hillertz, P., Pilon, M., and Enejder, A. (2007). Monitoring of lipid storage in Caenorhabditis elegans using coherent anti-Stokes Raman scattering (CARS) microscopy. Proc. Natl. Acad. Sci. U. S. A. 104, 14658-14663. Abstract Article

Henderson, S.T., and Johnson, T.E. (2001). daf-16 integrates developmental and environmental inputs to mediate aging in the nematode Caenorhabditis elegans. Curr. Biol. 11, 1975-1980. Abstract Article 
Henis-Korenblit, S., Zhang, P., Hansen, M., McCormick, M., Lee, S.J., Cary, M., and Kenyon, C. (2010). Insulin/IGF-1 signaling mutants reprogram ER stress response regulators to promote longevity. Proc. Natl. Acad. Sci. U. S. A. 107, 9730-9735. Abstract Article

Herndon, L.A., Schmeissner, P.J., Dudaronek, J.M., Brown, P.A., Listner, K.M., Sakano, Y., Paupard, M.C., Hall, D.H., and Driscoll, M. (2002). Stochastic and genetic factors influence tissue-specific decline in ageing C. elegans. Nature 419, 808-814. Abstract Article

Hertweck, M., Gobel, C., and Baumeister, R. (2004). C. elegans SGK-1 is the critical component in the Akt/PKB kinase complex to control stress response and life span. Dev. Cell 6, 577-588. Abstract Article

Hollander, M.C., Blumenthal, G.M., and Dennis, P.A. (2011). PTEN loss in the continuum of common cancers, rare syndromes and mouse models. Nat. Rev. Cancer 11, 289-301. Abstract Article

Holzenberger, M., Dupont, J., Ducos, B., Leneuve, P., Geloen, A., Even, P.C., Cervera, P., and Le Bouc, Y. (2003). IGF-1 receptor regulates lifespan and resistance to oxidative stress in mice. Nature 421, 182-187. Abstract Article

Honda, Y., and Honda, S. (1999). The daf-2 gene network for longevity regulates oxidative stress resistance and Mn-superoxide dismutase gene expression in Caenorhabditis elegans. FASEB J. 13, 1385-1393. Abstract

Honda, Y., and Honda, S. (2002). Oxidative stress and life span determination in the nematode Caenorhabditis elegans. Ann. N. Y. Acad. Sci. 959, 466-474. Abstract Article

Honjoh, S., Yamamoto, T., Uno, M., and Nishida, E. (2009). Signalling through RHEB-1 mediates intermittent fasting-induced longevity in C. elegans. Nature 457, 726-730. Abstract Article

Hristova, M., Birse, D., Hong, Y., and Ambros, V. (2005). The Caenorhabditis elegans heterochronic regulator LIN-14 is a novel transcription factor that controls the developmental timing of transcription from the insulin/insulin-like growth factor gene ins-33 by direct DNA binding. Mol. Cell. Biol. 25, 11059-11072. Abstract Article

Hsin, H., and Kenyon, C. (1999). Signals from the reproductive system regulate the lifespan of C. elegans. Nature 399, 362-366. Abstract Article

Hsu, A.L., Feng, Z., Hsieh, M.Y., and Xu, X.Z. (2009). Identification by machine vision of the rate of motor activity decline as a lifespan predictor in C. elegans. Neurobiol. Aging 30,1498-1503. Abstract Article

Hsu, A.L., Murphy, C.T., and Kenyon, C. (2003). Regulation of aging and age-related disease by DAF-16 and heat-shock factor. Science 300, 1142-1145. Abstract Article

Hsu, S.Y., Nakabayashi, K., Nishi, S., Kumagai, J., Kudo, M., Sherwood, O.D., and Hsueh, A.J. (2002). Activation of orphan receptors by the hormone relaxin. Science 295, 671-674. Abstract Article

$\mathrm{Hu}$, P.J. (2007). Dauer, WormBook, ed. The C. elegans Research Community, WormBook, doi/10.1895/wormbook.1.144.1, http://www.wormbook.org.

Hu, P.J., Xu, J., and Ruvkun, G. (2006). Two membrane-associated tyrosine phosphatase homologs potentiate $C$. elegans AKT-1/PKB signaling. PLoS Genet. 2, e99. Abstract Article

Hua, Q.X., Nakagawa, S.H., Wilken, J., Ramos, R.R., Jia, W., Bass, J., and Weiss, M.A. (2003). A divergent INS protein in Caenorhabditis elegans structurally resembles human insulin and activates the human insulin receptor. Genes Dev. 17, 826-831. Abstract Article

Huang, C., Xiong, C., and Kornfeld, K. (2004). Measurements of age-related changes of physiological processes that predict lifespan of Caenorhabditis elegans. Proc. Natl. Acad. Sci. U. S. A. 101, 8084-8089. Abstract Article

Huang, H., Regan, K.M., Wang, F., Wang, D., Smith, D.I., van Deursen, J.M., and Tindall, D.J. (2005). Skp2 inhibits FOXO1 in tumor suppression through ubiquitin-mediated degradation. Proc. Natl. Acad. Sci. U. S. A. 102, 1649-1654. Abstract Article 
Hughes, S.E., Evason, K., Xiong, C., and Kornfeld, K. (2007). Genetic and pharmacological factors that influence reproductive aging in nematodes. PLoS Genet. 3, e25. Abstract Article

Hunt-Newbury, R., Viveiros, R., Johnsen, R., Mah, A., Anastas, D., Fang, L., Halfnight, E., Lee, D., Lin, J., Lorch, A., et al. (2007). High-throughput in vivo analysis of gene expression in Caenorhabditis elegans. PLoS Biol. 5, e237. Abstract Article

Jenkins, N.L., McColl, G., and Lithgow, G.J. (2004). Fitness cost of extended lifespan in Caenorhabditis elegans. Proc. Biol. Sci. 271, 2523-2526. Abstract Article

Jia, K., Albert, P.S., and Riddle, D.L. (2002). DAF-9, a cytochrome P450 regulating C. elegans larval development and adult longevity. Development 129, 221-231. Abstract

Jia, K., Chen, D., and Riddle, D.L. (2004). The TOR pathway interacts with the insulin signaling pathway to regulate C. elegans larval development, metabolism and life span. Development 131, 3897-3906. Abstract Article

Johnson, T.E. (1990). Increased life-span of age-1 mutants in Caenorhabditis elegans and lower Gompertz rate of aging. Science 249, 908-912. Abstract Article

Jones, K.T., Greer, E.R., Pearce, D., and Ashrafi, K. (2009). Rictor/TORC2 regulates Caenorhabditis elegans fat storage, body size, and development through sgk-1. PLoS Biol. 7, e60. Abstract Article

Kahn, N.W., Rea, S.L., Moyle, S., Kell, A., and Johnson, T.E. (2008). Proteasomal dysfunction activates the transcription factor SKN-1 and produces a selective oxidative-stress response in Caenorhabditis elegans. Biochem. J. 409, 205-213. Abstract Article

Kao, G., Nordenson, C., Still, M., Ronnlund, A., Tuck, S., and Naredi, P. (2007). ASNA-1 positively regulates insulin secretion in C. elegans and mammalian cells. Cell 128, 577-587. Abstract Article

Karp, X., and Greenwald, I. (2013). Control of cell-fate plasticity and maintenance of multipotency by DAF-16/FoxO in quiescent Caenorhabditis elegans. Proc. Natl. Acad. Sci. U. S. A. 110, 2181-2186. Abstract Article

Kato, S., Ding, J., Pisck, E., Jhala, U.S., and Du, K. (2008). COP1 functions as a FoxO1 ubiquitin E3 ligase to regulate FoxO1-mediated gene expression. J. Biol. Chem. 283, 35464-35473. Abstract Article

Kauffman, A.L., Ashraf, J.M., Corces-Zimmerman, M.R., Landis, J.N., and Murphy, C.T. (2010). Insulin signaling and dietary restriction differentially influence the decline of learning and memory with age. PLoS Biol. 8, e1000372. Abstract Article

Kawano, T., Ito, Y., Ishiguro, M., Takuwa, K., Nakajima, T., and Kimura, Y. (2000). Molecular cloning and characterization of a new insulin/IGF-like peptide of the nematode Caenorhabditis elegans. Biochem. Biophys. Res. Commun. 273, 431-436. Abstract Article

Kenyon, C., Chang, J., Gensch, E., Rudner, A., and Tabtiang, R. (1993). A C. elegans mutant that lives twice as long as wild type. Nature 366, 461-464. Abstract

Keowkase, R., Aboukhatwa, M., and Luo, Y. (2010). Fluoxetine protects against amyloid- $\beta$ toxicity, in part via daf-16 mediated cell signaling pathway, in Caenorhabditis elegans. Neuropharmacology 59, 358-365. Abstract Article

Kerry, S., TeKippe, M., Gaddis, N.C., and Aballay, A. (2006). GATA transcription factor required for immunity to bacterial and fungal pathogens. PLoS One 1, e77. Abstract Article

Kido, Y., Nakae, J., and Accili, D. (2001). Clinical review 125: The insulin receptor and its cellular targets. J. Clin. Endocrinol. Metab. 86, 972-979. Abstract Article

Kimura, K.D., Riddle, D.L., and Ruvkun, G. (2011). The C. elegans DAF-2 insulin-like receptor is abundantly expressed in the nervous system and regulated by nutritional status. Cold Spring Harb. Symp. Quant. Biol. 76, 113-120. Abstract Article 
Kimura, K.D., Tissenbaum, H.A., Liu, Y., and Ruvkun, G. (1997). daf-2, an insulin receptor-like gene that regulates longevity and diapause in Caenorhabditis elegans. Science 277, 942-946. Abstract Article

Klass, M., and Hirsh, D. (1976). Non-ageing developmental variant of Caenorhabditis elegans. Nature 260, 523-525. Abstract Article

Klass, M.R. (1983). A method for the isolation of longevity mutants in the nematode Caenorhabditis elegans and initial results. Mech. Ageing Dev. 22, 279-286. Abstract Article

Kornfeld, K., Hom, D.B., and Horvitz, H.R. (1995). The ksr-1 gene encodes a novel protein kinase involved in Ras-mediated signaling in C. elegans. Cell 83, 903-913. Abstract Article

Kwon, E.S., Narasimhan, S.D., Yen, K., and Tissenbaum, H.A. (2010). A new DAF-16 isoform regulates longevity. Nature 466, 498-502. Abstract Article

Lamitina, S.T., and Strange, K. (2005). Transcriptional targets of DAF-16 insulin signaling pathway protect $C$. elegans from extreme hypertonic stress. Am. J. Physiol. Cell Physiol. 288, C467-474. Abstract Article

Landis, J.N., and Murphy, C.T. (2010). Integration of diverse inputs in the regulation of Caenorhabditis elegans DAF-16/FOXO. Dev. Dyn. 239, 1405-1412. Abstract Article

Lapierre, L.R., Gelino, S., Melendez, A., and Hansen, M. (2011). Autophagy and lipid metabolism coordinately modulate life span in germline-less C. elegans. Curr. Biol. 21, 1507-1514. Abstract Article

Lapierre, L.R., Melendez, A., and Hansen, M. (2012). Autophagy links lipid metabolism to longevity in C. elegans. Autophagy 8, 144-146. Abstract Article

Larsen, P.L., Albert, P.S., and Riddle, D.L. (1995). Genes that regulate both development and longevity in Caenorhabditis elegans. Genetics 139, 1567-1583. Abstract

LaRue, B.L., and Padilla, P.A. (2011). Environmental and genetic preconditioning for long-term anoxia responses requires AMPK in Caenorhabditis elegans. PLoS One 6, e16790. Abstract Article

Lawrence, M.C., McKern, N.M., and Ward, C.W. (2007). Insulin receptor structure and its implications for the IGF-1 receptor. Curr. Opin. Struct. Biol. 17, 699-705. Abstract Article

Lee, B.H., Liu, J., Wong, D., Srinivasan, S., and Ashrafi, K. (2011). Hyperactive neuroendocrine secretion causes size, feeding, and metabolic defects of C. elegans Bardet-Biedl syndrome mutants. PLoS Biol. 9, e1001219. Abstract Article

Lee, H., Cho, J.S., Lambacher, N., Lee, J., Lee, S.J., Lee, T.H., Gartner, A., and Koo, H.S. (2008). The Caenorhabditis elegans AMP-activated protein kinase AAK-2 is phosphorylated by LKB1 and is required for resistance to oxidative stress and for normal motility and foraging behavior. J. Biol. Chem. 283, 14988-14993. Abstract Article

Lee, R.C., Feinbaum, R.L., and Ambros, V. (1993). The C. elegans heterochronic gene lin-4 encodes small RNAs with antisense complementarity to lin-14. Cell 75, 843-854. Abstract

Lee, R.Y., Hench, J., and Ruvkun, G. (2001). Regulation of C. elegans DAF-16 and its human ortholog FKHRL1 by the daf-2 insulin-like signaling pathway. Curr. Biol. 11, 1950-1957. Abstract

Lee, S.J., Murphy, C.T., and Kenyon, C. (2009). Glucose shortens the life span of C. elegans by downregulating DAF-16/FOXO activity and aquaporin gene expression. Cell Metab. 10, 379-391. Abstract Article

Lee, S.S., Kennedy, S., Tolonen, A.C., and Ruvkun, G. (2003). DAF-16 target genes that control C. elegans life-span and metabolism. Science 300, 644-647. Abstract Article

Lehtinen, M.K., Yuan Z., Boag P. R., Yang Y.,Villén J., Becker, E. B.E., DiBacco, S., de la Iglesia, N., Gygi, S., Blackwell, T. K, and Bonni, A. (2006). A conserved MST-FOXO signaling pathway mediates oxidative-stress responses and extends life span. Cell 125, 987-1001. Abstract Article 
Li, J., Ebata, A., Dong, Y., Rizki, G., Iwata, T., and Lee, S.S. (2008). Caenorhabditis elegans HCF-1 functions in longevity maintenance as a DAF-16 regulator. PLoS Biol. 6, e233. Abstract Article

Li, J., Tewari, M., Vidal, M., and Lee, S.S. (2007a). The 14-3-3 protein FTT-2 regulates DAF-16 in Caenorhabditis elegans. Dev. Biol. 301, 82-91. Abstract Article

Li, W., Gao, B., Lee, S.M., Bennett, K., and Fang, D. (2007b). RLE-1, an E3 ubiquitin ligase, regulates C. elegans aging by catalyzing DAF-16 polyubiquitination. Dev. Cell 12, 235-246. Abstract Article

Li, W., Kennedy, S.G., and Ruvkun, G. (2003). daf-28 encodes a C. elegans insulin superfamily member that is regulated by environmental cues and acts in the DAF-2 signaling pathway. Genes Dev. 17, 844-858. Abstract Article

Li, X., Matilainen, O., Jin, C., Glover-Cutter, K.M., Holmberg, C.I., and Blackwell, T.K. (2011). Specific SKN-1/Nrf stress responses to perturbations in translation elongation and proteasome activity. PLoS Genet. 7, e1002119. Abstract Article

Libina, N., Berman, J.R., and Kenyon, C. (2003). Tissue-specific activities of C. elegans DAF-16 in the regulation of lifespan. Cell 115, 489-502. Abstract Article

Lin, C.H., Tomioka, M., Pereira, S., Sellings, L., Iino, Y., and van der Kooy, D. (2010). Insulin signaling plays a dual role in Caenorhabditis elegans memory acquisition and memory retrieval. J. Neurosci. 30, 8001-8011. Abstract

Lin, K., Dorman, J.B., Rodan, A., and Kenyon, C. (1997). daf-16: An HNF-3/forkhead family member that can function to double the life-span of Caenorhabditis elegans. Science 278, 1319-1322. Abstract Article

Lin, K., Hsin, H., Libina, N., and Kenyon, C. (2001). Regulation of the Caenorhabditis elegans longevity protein DAF-16 by insulin/IGF-1 and germline signaling. Nat. Genet. 28, 139-145. Abstract Article

Lithgow, G.J., White, T.M., Hinerfeld, D.A., and Johnson, T.E. (1994). Thermotolerance of a long-lived mutant of Caenorhabditis elegans. J. Gerontol. 49, B270-276. Abstract Article

Lithgow, G.J., White, T.M., Melov, S., and Johnson, T.E. (1995). Thermotolerance and extended life-span conferred by single-gene mutations and induced by thermal stress. Proc. Natl. Acad. Sci. U. S. A. 92, 7540-7544. Abstract

Liu, J., Kimura, A., Baumann, C.A., and Saltiel, A.R. (2002). APS facilitates c-Cbl tyrosine phosphorylation and GLUT4 translocation in response to insulin in 3T3-L1 adipocytes. Mol. Cell. Biol. 22, 3599-3609. Abstract Article

Liu, T., Zimmerman, K.K., and Patterson, G.I. (2004). Regulation of signaling genes by TGF- $\beta$ during entry into dauer diapause in C. elegans. BMC Dev. Biol. 4, 11. Abstract

Luo, R.Z., Beniac, D.R., Fernandes, A., Yip, C.C., and Ottensmeyer, F.P. (1999). Quaternary structure of the insulin-insulin receptor complex. Science 285, 1077-1080. Abstract Article

Luo, S., Kleemann, G.A., Ashraf, J.M., Shaw, W.M., and Murphy, C.T. (2010). TGF- $\beta$ and insulin signaling regulate reproductive aging via oocyte and germline quality maintenance. Cell 143, 299-312. Abstract

Luo, S., and Murphy, C.T. (2011). Caenorhabditis elegans reproductive aging: Regulation and underlying mechanisms. Genesis 49, 53-65. Abstract Article

Luo, S., Shaw, W.M., Ashraf, J., and Murphy, C.T. (2009). TGF- $\beta$ Sma/Mab signaling mutations uncouple reproductive aging from somatic aging. PLoS Genet. 5, e1000789. Abstract Article

Luzi, L., Confalonieri, S., Di Fiore, P.P., and Pelicci, P.G. (2000). Evolution of Shc functions from nematode to human. Curr. Opin. Genet. Dev. 10, 668-674. Abstract Article

Mair, W., Morantte, I., Rodrigues, A.P., Manning, G., Montminy, M., Shaw, R.J., and Dillin, A. (2011). Lifespan extension induced by AMPK and calcineurin is mediated by CRTC-1 and CREB. Nature 470, 404-408. Abstract Article 
Mak, H.Y., and Ruvkun, G. (2004). Intercellular signaling of reproductive development by the C. elegans DAF-9 cytochrome P450. Development 131, 1777-1786. Abstract Article

Malone, E.A., Inoue, T., and Thomas, J.H. (1996). Genetic analysis of the roles of daf-28 and age-1 in regulating Caenorhabditis elegans dauer formation. Genetics 143, 1193-1205. Abstract

Manning, B.D., and Cantley, L.C. (2007). AKT/PKB signaling: navigating downstream. Cell 129, 1261-1274. Abstract Article

Matsuzaki, H., Daitoku, H., Hatta, M., Tanaka, K., and Fukamizu, A. (2003). Insulin-induced phosphorylation of FKHR (Foxo1) targets to proteasomal degradation. Proc. Natl. Acad. Sci. U. S. A. 100, 11285-11290. Abstract Article

McCormick, M., Chen, K., Ramaswamy, P., and Kenyon, C. (2011). New genes that extend Caenorhabditis elegans' lifespan in response to reproductive signals. Aging Cell 11, 192-202. Abstract Article

McElwee, J., Bubb, K., and Thomas, J.H. (2003). Transcriptional outputs of the Caenorhabditis elegans forkhead protein DAF-16. Aging Cell 2, 111-121. Abstract Article

McElwee, J.J., Schuster, E., Blanc, E., Thomas, J.H., and Gems, D. (2004). Shared transcriptional signature in Caenorhabditis elegans Dauer larvae and long-lived daf-2 mutants implicates detoxification system in longevity assurance. J. Biol. Chem. 279, 44533-44543. Abstract Article

Melendez, A., Talloczy, Z., Seaman, M., Eskelinen, E.L., Hall, D.H., and Levine, B. (2003). Autophagy genes are essential for dauer development and life-span extension in C. elegans. Science 301, 1387-1391. Abstract

Mendoza, M.C., Du, F., Iranfar, N., Tang, N., Ma, H., Loomis, W.F., and Firtel, R.A. (2005). Loss of SMEK, a novel, conserved protein, suppresses MEK1 null cell polarity, chemotaxis, and gene expression defects. Mol. Cell. Biol. 25, 7839-7853. Abstract Article

Meyer, B. J. (2005). X-Chromosome dosage compensation, WormBook, ed. The C. elegans Research Community, WormBook, doi/10.1895/wormbook.1.8.1, http://www.wormbook.org.

Meyer, B.J. (2010). Targeting X chromosomes for repression. Curr. Opin. Genet. Dev. 20, 179-189. Abstract Article

Michaelson, D., Korta, D.Z., Capua, Y., and Hubbard, E.J. (2010). Insulin signaling promotes germline proliferation in C. elegans. Development 137, 671-680. Abstract Article

Mihaylova, M.M., Vasquez, D.S., Ravnskjaer, K., Denechaud, P.D., Yu, R.T., Alvarez, J.G., Downes, M., Evans, R.M., Montminy, M., and Shaw, R.J. (2011). Class IIa histone deacetylases are hormone-activated regulators of FOXO and mammalian glucose homeostasis. Cell 145, 607-621. Abstract Article

Mihaylova, V.T., Borland, C.Z., Manjarrez, L., Stern, M.J., and Sun, H. (1999). The PTEN tumor suppressor homolog in Caenorhabditis elegans regulates longevity and dauer formation in an insulin receptor-like signaling pathway. Proc. Natl. Acad. Sci. U. S. A. 96, 7427-7432. Abstract Article

Mizuno, T., Fujiki, K., Sasakawa, A., Hisamoto, N., and Matsumoto, K. (2008). Role of the Caenorhabditis elegans Shc adaptor protein in the c-Jun N-terminal kinase signaling pathway. Mol. Cell. Biol. 28, 7041-7049. Abstract Article

Morley, J.F., Brignull, H.R., Weyers, J.J., and Morimoto, R.I. (2002). The threshold for polyglutamine-expansion protein aggregation and cellular toxicity is dynamic and influenced by aging in Caenorhabditis elegans. Proc. Natl. Acad. Sci. U. S. A.. Abstract Article

Morley, J.F., and Morimoto, R.I. (2004). Regulation of longevity in Caenorhabditis elegans by heat shock factor and molecular chaperones. Mol. Biol. Cell 15, 657-664. Abstract Article

Morris, J.Z., Tissenbaum, H.A., and Ruvkun, G. (1996). A phosphatidylinositol-3-OH kinase family member regulating longevity and diapause in Caenorhabditis elegans. Nature 382, 536-539. Abstract Article 
Moss, E.G., Lee, R.C., and Ambros, V. (1997). The cold shock domain protein LIN-28 controls developmental timing in C. elegans and is regulated by the lin-4 RNA. Cell 88, 637-646. Abstract Article

Motola, D.L., Cummins, C.L., Rottiers, V., Sharma, K.K., Li, T., Li, Y., Suino-Powell, K., Xu, H.E., Auchus, R.J., Antebi, A., et al. (2006). Identification of ligands for DAF-12 that govern dauer formation and reproduction in $C$. elegans. Cell 124, 1209-1223. Abstract Article

Motta, M.C., Divecha, N., Lemieux, M., Kamel, C., Chen, D., Gu, W., Bultsma, Y., McBurney, M., and Guarente, L. (2004). Mammalian SIRT1 represses forkhead transcription factors. Cell 116, 551-563. Abstract Article

Munoz, M.J., and Riddle, D.L. (2003). Positive selection of Caenorhabditis elegans mutants with increased stress resistance and longevity. Genetics 163, 171-180. Abstract

Murakami, H., Bessinger, K., Hellmann, J., and Murakami, S. (2005). Aging-dependent and -independent modulation of associative learning behavior by insulin/insulin-like growth factor-1 signal in Caenorhabditis elegans. J. Neurosci. 25, 10894-10904. Abstract

Murakami, S., and Johnson, T.E. (1996). A genetic pathway conferring life extension and resistance to UV stress in Caenorhabditis elegans. Genetics 143, 1207-1218. Abstract

Murphy, C.T. (2006). The search for DAF-16/FOXO transcriptional targets: Approaches and discoveries. Exp. Gerontol. 41, 910-921. Abstract Article

Murphy, C.T., Lee, S.J., and Kenyon, C. (2007). Tissue entrainment by feedback regulation of insulin gene expression in the endoderm of Caenorhabditis elegans. Proc. Natl. Acad. Sci. U. S. A. 104, 19046-19050. Abstract Article

Murphy, C.T., McCarroll, S.A., Bargmann, C.I., Fraser, A., Kamath, R.S., Ahringer, J., Li, H., and Kenyon, C. (2003). Genes that act downstream of DAF-16 to influence the lifespan of Caenorhabditis elegans. Nature 424, 277-283. Abstract Article

Myers, M.P., Pass, I., Batty, I.H., Van der Kaay, J., Stolarov, J.P., Hemmings, B.A., Wigler, M.H., Downes, C.P., and Tonks, N.K. (1998). The lipid phosphatase activity of PTEN is critical for its tumor supressor function. Proc. Natl. Acad. Sci. U. S. A. 95, 13513-13518. Abstract Article

Nakdimon, I., Walser, M., Frohli, E., and Hajnal, A. (2012). PTEN negatively regulates MAPK signaling during Caenorhabditis elegans vulval development. PLoS Genet. 8, e1002881. Abstract Article

Nanji, M., Hopper, N.A., and Gems, D. (2005). LET-60 RAS modulates effects of insulin/IGF-1 signaling on development and aging in Caenorhabditis elegans. Aging Cell 4, 235-245. Abstract Article

Narasimhan, S.D., Yen, K., Bansal, A., Kwon, E.S., Padmanabhan, S., and Tissenbaum, H.A. (2011). PDP-1 links the TGF- $\beta$ and IIS pathways to regulate longevity, development, and metabolism. PLoS Genet. 7, e1001377. Abstract Article

Narbonne, P., and Roy, R. (2006). Inhibition of germline proliferation during C. elegans dauer development requires PTEN, LKB1 and AMPK signalling. Development 133, 611-619. Abstract Article

Narbonne, P., and Roy, R. (2009). Caenorhabditis elegans dauers need LKB1/AMPK to ration lipid reserves and ensure long-term survival. Nature 457, 210-214. Abstract Article

Nasrin, N., Ogg, S., Cahill, C.M., Biggs, W., Nui, S., Dore, J., Calvo, D., Shi, Y., Ruvkun, G., and Alexander-Bridges, M.C. (2000). DAF-16 recruits the CREB-binding protein coactivator complex to the insulin-like growth factor binding protein 1 promoter in HepG2 cells. Proc. Natl. Acad. Sci. U. S. A. 97, 10412-10417. Abstract Article

Neumann-Haefelin, E., Qi, W., Finkbeiner, E., Walz, G., Baumeister, R., and Hertweck, M. (2008). SHC-1/p52Shc targets the insulin/IGF-1 and JNK signaling pathways to modulate life span and stress response in C. elegans. Genes Dev. 22, 2721-2735. Abstract Article 
Niu, W., Lu, Z.J., Zhong, M., Sarov, M., Murray, J.I., Brdlik, C.M., Janette, J., Chen, C., Alves, P., Preston, E., et al. (2011). Diverse transcription factor binding features revealed by genome-wide ChIP-seq in C. elegans. Genome Res. 21, 245-254. Abstract Article

O'Rourke, E.J., Soukas, A.A., Carr, C.E., and Ruvkun, G. (2009). C. elegans major fats are stored in vesicles distinct from lysosome-related organelles. Cell Metab. 10, 430-435. Abstract Article

Obsil, T., and Obsilova, V. (2008). Structure/function relationships underlying regulation of FOXO transcription factors. Oncogene 27, 2263-2275. Abstract Article

Oda, S., Tomioka, M., and Iino, Y. (2011). Neuronal plasticity regulated by the insulin-like signaling pathway underlies salt chemotaxis learning in Caenorhabditis elegans. J. Neurophysiol. 106, 301-308. Abstract Article

Ogg, S., Paradis, S., Gottlieb, S., Patterson, G.I., Lee, L., Tissenbaum, H.A., and Ruvkun, G. (1997). The Fork head transcription factor DAF-16 transduces insulin-like metabolic and longevity signals in C. elegans. Nature 389, 994-999. Abstract Article

Ogg, S., and Ruvkun, G. (1998). The C. elegans PTEN homolog, DAF-18, acts in the insulin receptor-like metabolic signaling pathway. Mol. Cell 2, 887-893. Abstract Article

Oh, S.W., Mukhopadhyay, A., Dixit, B.L., Raha, T., Green, M.R., and Tissenbaum, H.A. (2006). Identification of direct DAF-16 targets controlling longevity, metabolism and diapause by chromatin immunoprecipitation. Nat. Genet. 38, 251-257. Abstract Article

Oh, S.W., Mukhopadhyay, A., Svrzikapa, N., Jiang, F., Davis, R.J., and Tissenbaum, H.A. (2005). JNK regulates lifespan in Caenorhabditis elegans by modulating nuclear translocation of forkhead transcription factor/DAF-16. Proc. Natl. Acad. Sci. U. S. A. 102, 4494-4499. Abstract Article

Ohkura, K., Suzuki, N., Ishihara, T., and Katsura, I. (2003). SDF-9, a protein tyrosine phosphatase-like molecule, regulates the L3/dauer developmental decision through hormonal signaling in C. elegans. Development 130, 3237-3248. Abstract Article

Oliveira, R.P., Porter Abate, J., Dilks, K., Landis, J., Ashraf, J., Murphy, C.T., and Blackwell, T.K. (2009). Condition-adapted stress and longevity gene regulation by Caenorhabditis elegans SKN-1/Nrf. Aging Cell 8, 524-541. Abstract Article

Olsen, P.H., and Ambros, V. (1999). The lin-4 regulatory RNA controls developmental timing in Caenorhabditis elegans by blocking LIN-14 protein synthesis after the initiation of translation. Dev. Biol. 216, 671-680. Abstract Article

Ookuma, S., Fukuda, M., and Nishida, E. (2003). Identification of a DAF-16 transcriptional target gene, scl-1, that regulates longevity and stress resistance in Caenorhabditis elegans. Curr. Biol. 13, 427-431. Abstract Article

Padmanabhan, S., Mukhopadhyay, A., Narasimhan, S.D., Tesz, G., Czech, M.P., and Tissenbaum, H.A. (2009). A PP2A regulatory subunit regulates C. elegans insulin/IGF-1 signaling by modulating AKT-1 phosphorylation. Cell 136, 939-951. Abstract Article

Paek, J., Lo, J.Y., Narasimhan, S.D., Nguyen, T.N., Glover-Cutter, K., Robida-Stubbs, S., Suzuki, T., Yamamoto, M., Blackwell, T.K., and Curran, S.P. (2012). Mitochondrial SKN-1/Nrf mediates a conserved starvation response. Cell Metab. 16, 526-537. Abstract Article

Pan, C.L., Peng, C.Y., Chen, C.H., and McIntire, S. (2011). Genetic analysis of age-dependent defects of the Caenorhabditis elegans touch receptor neurons. Proc. Natl. Acad. Sci. U. S. A. 108, 9274-9279. Abstract Article

Panowski, S.H., Wolff, S., Aguilaniu, H., Durieux, J., and Dillin, A. (2007). PHA-4/Foxa mediates diet-restriction-induced longevity of C. elegans. Nature 447, 550-555. Abstract Article

Paradis, S., Ailion, M., Toker, A., Thomas, J.H., and Ruvkun, G. (1999). A PDK1 homolog is necessary and sufficient to transduce AGE-1 PI3 kinase signals that regulate diapause in Caenorhabditis elegans. Genes Dev. 13, 1438-1452. Abstract 
Paradis, S., and Ruvkun, G. (1998). Caenorhabditis elegans Akt/PKB transduces insulin receptor-like signals from AGE-1 PI3 kinase to the DAF-16 transcription factor. Genes Dev. 12, 2488-2498. Abstract Article

Patel, D.S., Fang, L.L., Svy, D.K., Ruvkun, G., and Li, W. (2008a). Genetic identification of HSD-1, a conserved steroidogenic enzyme that directs larval development in Caenorhabditis elegans. Development 135, 2239-2249. Abstract Article

Patel, D.S., Garza-Garcia, A., Nanji, M., McElwee, J.J., Ackerman, D., Driscoll, P.C., and Gems, D. (2008b). Clustering of genetically defined allele classes in the Caenorhabditis elegans DAF-2 insulin/IGF-1 receptor. Genetics 178, 931-946. Abstract Article

Pearce, L.R., Komander, D., and Alessi, D.R. (2010). The nuts and bolts of AGC protein kinases. Nat. Rev. Mol. Cell Biol. 11, 9-22. Abstract Article

Perez, C.L., and Van Gilst, M.R. (2008). A ${ }^{13} \mathrm{C}$ isotope labeling strategy reveals the influence of insulin signaling on lipogenesis in C. elegans. Cell Metab. 8, 266-274. Abstract Article

Pierce, S.B., Costa, M., Wisotzkey, R., Devadhar, S., Homburger, S.A., Buchman, A.R., Ferguson, K.C., Heller, J., Platt, D.M., Pasquinelli, A.A., et al. (2001). Regulation of DAF-2 receptor signaling by human insulin and ins-1, a member of the unusually large and diverse C. elegans insulin gene family. Genes Dev. 15, 672-686. Abstract Article

Pinero Gonzalez, J., Carrillo Farnes, O., Vasconcelos, A.T., and Gonzalez Perez, A. (2009). Conservation of key members in the course of the evolution of the insulin signaling pathway. Biosystems 95, 7-16. Abstract Article

Pinkston-Gosse, J., and Kenyon, C. (2007). DAF-16/FOXO targets genes that regulate tumor growth in Caenorhabditis elegans. Nat. Genet. 39, 1403-1409. Abstract Article

Ravichandran, K.S. (2001). Signaling via Shc family adapter proteins. Oncogene 20, 6322-6330. Abstract Article

Ren, P., Lim, C.S., Johnsen, R., Albert, P.S., Pilgrim, D., and Riddle, D.L. (1996). Control of C. elegans larval development by neuronal expression of a TGF- $\beta$ homolog. Science 274, 1389-1391. Abstract Article

Riddle, D.L., Swanson, M.M., and Albert, P.S. (1981). Interacting genes in nematode dauer larva formation. Nature 290, 668-671. Abstract Article

Rizki, G., Iwata, T.N., Li, J., Riedel, C.G., Picard, C.L., Jan, M., Murphy, C.T., and Lee, S.S. (2011). The evolutionarily conserved longevity determinants HCF-1 and SIR-2.1/SIRT1 collaborate to regulate DAF-16/FOXO. PLoS Genet. 7, e1002235. Abstract Article

Robida-Stubbs, S., Glover-Cutter, K., Lamming, D.W., Mizunuma, M., Narasimhan, S.D., Neumann-Haefelin, E., Sabatini, D.M., and Blackwell, T.K. (2012). TOR signaling and rapamycin influence longevity by regulating SKN-1/Nrf and DAF-16/FoxO. Cell Metab. 15, 713-724. Abstract Article

Rodriguez-Escudero, I., Oliver, M.D., Andres-Pons, A., Molina, M., Cid, V.J., and Pulido, R. (2011). A comprehensive functional analysis of PTEN mutations: implications in tumor- and autism-related syndromes. Hum. Mol. Genet. 20, 4132-4142. Abstract Article

Rouault, J.P., Kuwabara, P.E., Sinilnikova, O.M., Duret, L., Thierry-Mieg, D., and Billaud, M. (1999). Regulation of dauer larva development in Caenorhabditis elegans by daf-18, a homologue of the tumour suppressor PTEN. Curr. Biol. 9, 329-332. Abstract Article

Ruaud, A.F., Katic, I., and Bessereau, J.L. (2011). Insulin/Insulin-like growth factor signaling controls non-Dauer developmental speed in the nematode Caenorhabditis elegans. Genetics 187, 337-343. Abstract Article

Sarbassov, D.D., Guertin, D.A., Ali, S.M., and Sabatini, D.M. (2005). Phosphorylation and regulation of Akt/PKB by the rictor-mTOR complex. Science 307, 1098-1101. Abstract Article

Saul, N., Pietsch, K., Menzel, R., Sturzenbaum, S.R., and Steinberg, C.E. (2009). Catechin induced longevity in $C$. elegans: From key regulator genes to disposable soma. Mech. Aging Dev. 130, 477-486. Abstract Article 
Schackwitz, W.S., Inoue, T., and Thomas, J.H. (1996). Chemosensory neurons function in parallel to mediate a pheromone response in C. elegans. Neuron 17, 719-728. Abstract Article

Schaedel, O.N., Gerisch, B., Antebi, A., and Sternberg, P.W. (2012). Hormonal signal amplification mediates environmental conditions during development and controls an irreversible commitment to adulthood. PLoS Biol. 10, e1001306. Abstract Article

Schuster, E., McElwee, J.J., Tullet, J.M., Doonan, R., Matthijssens, F., Reece-Hoyes, J.S., Hope, I.A., Vanfleteren, J.R., Thornton, J.M., and Gems, D. (2010). DamID in C. elegans reveals longevity-associated targets of DAF-16/FoxO. Mol. Syst. Biol. 6, 399. Abstract Article

Scott, B.A., Avidan, M.S., and Crowder, C.M. (2002). Regulation of hypoxic death in C. elegans by the insulin/IGF receptor homolog DAF-2. Science 296, 2388-2391. Abstract Article

Shaw, L.M. (2011). The insulin receptor substrate (IRS) proteins: At the intersection of metabolism and cancer. Cell Cycle 10, 1750-1756. Abstract Article

Shaw, W.M., Luo, S., Landis, J., Ashraf, J., and Murphy, C.T. (2007). The C. elegans TGF- $\beta$ Dauer pathway regulates longevity via insulin signaling. Curr. Biol. 17, 1635-1645. Abstract Article

Sheaffer, K.L., Updike, D.L., and Mango, S.E. (2008). The Target of Rapamycin pathway antagonizes pha-4/FoxA to control development and aging. Curr. Biol. 18, 1355-1364. Abstract Article

Shen, Y., Wollam, J., Magner, D., Karalay, O., and Antebi, A. (2012). A steroid receptor-microRNA switch regulates life span in response to signals from the gonad. Science 338, 1472-1476. Abstract Article

Siddle, K. (2011). Signalling by insulin and IGF receptors: supporting acts and new players. J. Mol. Endocrinol. 47, R1-10. Abstract Article

Solari, F., Bourbon-Piffaut, A., Masse, I., Payrastre, B., Chan, A.M., and Billaud, M. (2005). The human tumour suppressor PTEN regulates longevity and dauer formation in Caenorhabditis elegans. Oncogene 24, 20-27. Abstract Article

Soukas, A.A., Kane, E.A., Carr, C.E., Melo, J.A., and Ruvkun, G. (2009). Rictor/TORC2 regulates fat metabolism, feeding, growth, and life span in Caenorhabditis elegans. Genes Dev. 23, 496-511. Abstract Article

Stein, G.M., and Murphy, C.T. (2012). The intersection of aging, longevity pathways, and learning and memory in C. elegans. Front. Genet. 3, 259. Abstract Article

Suh, Y., Atzmon, G., Cho, M.O., Hwang, D., Liu, B., Leahy, D.J., Barzilai, N., and Cohen, P. (2008). Functionally significant insulin-like growth factor I receptor mutations in centenarians. Proc. Natl. Acad. Sci. U. S. A. 105, 3438-3442. Abstract Article

Sun, X.J., Rothenberg, P., Kahn, C.R., Backer, J.M., Araki, E., Wilden, P.A., Cahill, D.A., Goldstein, B.J., and White, M.F. (1991). Structure of the insulin receptor substrate IRS-1 defines a unique signal transduction protein. Nature 352, 73-77. Abstract

Sun, X.J., Wang, L.M., Zhang, Y., Yenush, L., Myers, M.G., Jr., Glasheen, E., Lane, W.S., Pierce, J.H., and White, M.F. (1995). Role of IRS-2 in insulin and cytokine signalling. Nature 377, 173-177. Abstract

Sundaram, M., and Han, M. (1995). The C. elegans ksr-1 gene encodes a novel Raf-related kinase involved in Ras-mediated signal transduction. Cell 83, 889-901. Abstract Article

Sundaram M.V. (2013). Canonical RTK-Ras-ERK signaling and related alternative pathways, WormBook, ed. The C. elegans Research Community, WormBook, doi/10.1895/wormbook.1.80.2, http://www.wormbook.org.

Szewczyk, N.J., Peterson, B.K., Barmada, S.J., Parkinson, L.P., and Jacobson, L.A. (2007). Opposed growth factor signals control protein degradation in muscles of Caenorhabditis elegans. EMBO J. 26, 935-943. Abstract Article 
Takahashi, Y., Daitoku, H., Hirota, K., Tamiya, H., Yokoyama, A., Kako, K., Nagashima, Y., Nakamura, A., Shimada, T., Watanabe, S., et al. (2011). Asymmetric arginine dimethylation determines life span in C. elegans by regulating forkhead transcription factor DAF-16. Cell Metab. 13, 505-516. Abstract Article

Tan, K.T., Luo, S.C., Ho, W.Z., and Lee, Y.H. (2011). Insulin/IGF-1 receptor signaling enhances biosynthetic activity and fat mobilization in the initial phase of starvation in adult male C. elegans. Cell Metab. 14, 390-402. Abstract Article

Taniguchi, C.M., Emanuelli, B., and Kahn, C.R. (2006). Critical nodes in signalling pathways: insights into insulin action. Nat. Rev. Mol. Cell Biol. 7, 85-96. Abstract Article

Tank, E.M., Rodgers, K.E., and Kenyon, C. (2011). Spontaneous age-related neurite branching in Caenorhabditis elegans. J. Neurosci. 31, 9279-9288. Abstract Article

Tatar, M., Kopelman, A., Epstein, D., Tu, M.P., Yin, C.M., and Garofalo, R.S. (2001). A mutant Drosophila insulin receptor homolog that extends life-span and impairs neuroendocrine function. Science 292, 107-110. Abstract Article

Tazearslan, C., Huang, J., Barzilai, N., and Suh, Y. (2011). Impaired IGF1R signaling in cells expressing longevity-associated human IGF1R alleles. Aging Cell 10, 551-554. Abstract Article

Teixeira-Castro, A., Ailion, M., Jalles, A., Brignull, H.R., Vilaca, J.L., Dias, N., Rodrigues, P., Oliveira, J.F., Neves-Carvalho, A., Morimoto, R.I., et al. (2011). Neuron-specific proteotoxicity of mutant ataxin-3 in C. elegans: rescue by the DAF-16 and HSF-1 pathways. Hum. Mol. Genet. 20, 2996-3009. Abstract Article

Teleman, A.A., Chen, Y.W., and Cohen, S.M. (2005). Drosophila Melted modulates FOXO and TOR activity. Dev. Cell 9, 271-281. Abstract Article

Tepper, R.G., Ashraf, J., Kaletsky, R., Kleemann, G.A., Murphy, C.T., and Bussemaker, H.J. (2013). PQM-1 complements DAF-16 as a key transcriptional regulator of DAF-2-mediated development and longevity. Cell 154, 676-690. Abstract Article

Therrien, M., Chang, H.C., Solomon, N.M., Karim, F.D., Wassarman, D.A., and Rubin, G.M. (1995). KSR, a novel protein kinase required for RAS signal transduction. Cell 83, 879-888. Abstract Article

Tissenbaum, H.A., and Guarente, L. (2001). Increased dosage of a sir-2 gene extends lifespan in Caenorhabditis elegans. Nature 410, 227-230. Abstract Article

Toker, A., and Newton, A.C. (2000). Cellular signaling: pivoting around PDK-1. Cell 103, 185-188. Abstract

Tomioka, M., Adachi, T., Suzuki, H., Kunitomo, H., Schafer, W.R., and Iino, Y. (2006). The insulin/PI 3-kinase pathway regulates salt chemotaxis learning in Caenorhabditis elegans. Neuron 51, 613-625. Abstract Article

Torayama, I., Ishihara, T., and Katsura, I. (2007). Caenorhabditis elegans integrates the signals of butanone and food to enhance chemotaxis to butanone. J. Neurosci. 27, 741-750. Abstract Article

Troemel, E.R., Chu, S.W., Reinke, V., Lee, S.S., Ausubel, F.M., and Kim, D.H. (2006). p38 MAPK regulates expression of immune response genes and contributes to longevity in C. elegans. PLoS Genet. 2, e183. Abstract Article

Tsai, W.C., Bhattacharyya, N., Han, L.Y., Hanover, J.A., and Rechler, M.M. (2003). Insulin inhibition of transcription stimulated by the forkhead protein Foxo1 is not solely due to nuclear exclusion. Endocrinology 144, 5615-5622. Abstract Article

Tullet, J.M., Hertweck, M., An, J.H., Baker, J., Hwang, J.Y., Liu, S., Oliveira, R.P., Baumeister, R., and Blackwell, T.K. (2008). Direct inhibition of the longevity-promoting factor SKN-1 by insulin-like signaling in C. elegans. Cell 132, 1025-1038. Abstract Article

Ullrich, A., Bell, J.R., Chen, E.Y., Herrera, R., Petruzzelli, L.M., Dull, T.J., Gray, A., Coussens, L., Liao, Y.C., Tsubokawa, M., et al. (1985). Human insulin receptor and its relationship to the tyrosine kinase family of oncogenes. Nature 313, 756-761. Abstract Article 
Ullrich, A., Gray, A., Tam, A.W., Yang-Feng, T., Tsubokawa, M., Collins, C., Henzel, W., Le Bon, T., Kathuria, S., Chen, E., et al. (1986). Insulin-like growth factor I receptor primary structure: comparison with insulin receptor suggests structural determinants that define functional specificity. EMBO J. 5, 2503-2512. Abstract

van der Horst, A., Tertoolen, L.G., de Vries-Smits, L.M., Frye, R.A., Medema, R.H., and Burgering, B.M. (2004). FOXO4 is acetylated upon peroxide stress and deacetylated by the longevity protein hSir2(SIRT1). J. Biol. Chem. 279, 28873-28879. Abstract Article

Vellai, T., McCulloch, D., Gems, D., and Kovacs, A.L. (2006). Effects of sex and insulin/insulin-like growth factor-1 signaling on performance in an associative learning paradigm in Caenorhabditis elegans. Genetics 174, 309-316. Abstract Article

Vellai, T., Takacs-Vellai, K., Zhang, Y., Kovacs, A.L., Orosz, L., and Muller, F. (2003). Genetics: Influence of TOR kinase on lifespan in C. elegans. Nature 426, 620. Abstract

Vereshchagina, N., Ramel, M.C., Bitoun, E., and Wilson, C. (2008). The protein phosphatase PP2A-B' subunit Widerborst is a negative regulator of cytoplasmic activated Akt and lipid metabolism in Drosophila. J. Cell Sci. 121, 3383-3392. Abstract Article

Viswanathan, M., and Guarente, L. (2011). Regulation of Caenorhabditis elegans lifespan by sir-2.1 transgenes. Nature 477, E1-2. Abstract Article

Vowels, J.J., and Thomas, J.H. (1992). Genetic analysis of chemosensory control of dauer formation in Caenorhabditis elegans. Genetics 130, 105-123. Abstract

Walker, D.W., McColl, G., Jenkins, N.L., Harris, J., and Lithgow, G.J. (2000). Evolution of lifespan in C. elegans. Nature 405, 296-297. Abstract Article

Walker, G.A., Walker, D.W., and Lithgow, G.J. (1998). Genes that determine both thermotolerance and rate of aging in Caenorhabditis elegans. Ann. N. Y. Acad. Sci. 851, 444-449. Abstract Article

Walker, G.A., White, T.M., McColl, G., Jenkins, N.L., Babich, S., Candido, E.P., Johnson, T.E., and Lithgow, G.J. (2001). Heat shock protein accumulation is upregulated in a long-lived mutant of Caenorhabditis elegans. J. Gerontol. A Biol. Sci. Med. Sci. 56, B281-287. Abstract Article

Wang, D., and Ruvkun, G. (2004). Regulation of Caenorhabditis elegans RNA interference by the daf-2 insulin stress and longevity signaling pathway. Cold Spring Harb. Symp. Quant. Biol. 69, 429-431. Abstract Article

Wang, J., and Kim, S.K. (2003). Global analysis of dauer gene expression in Caenorhabditis elegans. Development 130, 1621-1634. Abstract Article

Wang, M.C., Bohmann, D., and Jasper, H. (2005). JNK extends life span and limits growth by antagonizing cellular and organism-wide responses to insulin signaling. Cell 121, 115-125. Abstract Article

Wang, Y., Oh, S.W., Deplancke, B., Luo, J., Walhout, A.J., and Tissenbaum, H.A. (2006). C. elegans 14-3-3 proteins regulate life span and interact with SIR-2.1 and DAF-16/FOXO. Mech. Ageing Dev. 127, 741-747. Abstract Article

White, M.F. (1998). The IRS-signalling system: a network of docking proteins that mediate insulin action. Mol. Cell. Biochem. 182, 3-11. Abstract

Wightman, B., Ha, I., and Ruvkun, G. (1993). Posttranscriptional regulation of the heterochronic gene lin-14 by lin-4 mediates temporal pattern formation in C. elegans. Cell 75, 855-862. Abstract Article

Willcox, B.J., Donlon, T.A., He, Q., Chen, R., Grove, J.S., Yano, K., Masaki, K.H., Willcox, D.C., Rodriguez, B., and Curb, J.D. (2008). FOXO3A genotype is strongly associated with human longevity. Proc. Natl. Acad. Sci. U. S. A. 105, 13987-13992. Abstract Article

Wilson, A.C., Cleary, M.A., Lai, J.S., LaMarco, K., Peterson, M.G., and Herr, W. (1993). Combinatorial control of transcription: the herpes simplex virus VP16-induced complex. Cold Spring Harb. Symp. Quant. Biol. 58, 167-178. Abstract Article 
Wolff, S., Ma, H., Burch, D., Maciel, G.A., Hunter, T., and Dillin, A. (2006). SMK-1, an essential regulator of DAF-16-mediated longevity. Cell 124, 1039-1053. Abstract Article

Wolkow, C.A., Kimura, K.D., Lee, M.S., and Ruvkun, G. (2000). Regulation of C. elegans life-span by insulinlike signaling in the nervous system. Science 290, 147-150. Abstract Article

Wolkow, C.A., Munoz, M.J., Riddle, D.L., and Ruvkun, G. (2002). IRS and p55 orthologous adaptor proteins function in the C. elegans daf-2/insulin-like signaling pathway. J. Biol. Chem. 277, 49591-49597. Abstract Article

Wollam, J., Magomedova, L., Magner, D.B., Shen, Y., Rottiers, V., Motola, D.L., Mangelsdorf, D.J., Cummins, C.L., and Antebi, A. (2011). The Rieske oxygenase DAF-36 functions as a cholesterol 7-desaturase in steroidogenic pathways governing longevity. Aging Cell 10, 879-884. Abstract Article

Xiao, R., Zhang, B., Dong, Y., Gong, J., Xu, T., Liu, J., and Xu, X.Z. (2013). A genetic program promotes $C$. elegans longevity at cold temperatures via a thermosensitive TRP channel. Cell 152, 806-817. Abstract Article

Yamagata, K., Daitoku, H., Takahashi, Y., Namiki, K., Hisatake, K., Kako, K., Mukai, H., Kasuya, Y., and Fukamizu, A. (2008). Arginine methylation of FOXO transcription factors inhibits their phosphorylation by Akt. Mol. Cell 32, 221-231. Abstract Article

Yamawaki, T.M., Arantes-Oliveira, N., Berman, J.R., Zhang, P., and Kenyon, C. (2008). Distinct activities of the germline and somatic reproductive tissues in the regulation of Caenorhabditis elegans" longevity. Genetics 178, 513-526. Abstract Article

Yamawaki, T.M., Berman, J.R., Suchanek-Kavipurapu, M., McCormick, M., Gaglia, M.M., Lee, S.J., and Kenyon, C. (2010). The somatic reproductive tissues of $C$. elegans promote longevity through steroid hormone signaling. PLoS Biol. 8, e1000468. Abstract Article

Yanase, S., Yasuda, K., and Ishii, N. (2002). Adaptive responses to oxidative damage in three mutants of Caenorhabditis elegans (age-1, mev-1 and daf-16) that affect life span. Mech. Ageing Dev. 123, 1579-1587. Abstract Article

Yang, J., Cron, P., Good, V.M., Thompson, V., Hemmings, B.A., and Barford, D. (2002). Crystal structure of an activated Akt/protein kinase B ternary complex with GSK3-peptide and AMP-PNP. Nat. Struct. Biol. 9, 940-944. Abstract Article

Yochem, J.K. (2006). Nomarski images for learning the anatomy, with tips for mosaic analysis, WormBook, ed. The C. elegans Research Community, WormBook, doi/10.1895/wormbook.1.100.1, http://www.wormbook.org.

Zaghloul, N.A., and Katsanis, N. (2009). Mechanistic insights into Bardet-Biedl syndrome, a model ciliopathy. J. Clin. Invest. 119, 428-437. Abstract Article

Zhang, T., Hwang, H.Y., Hao, H., Talbot, C., Jr., and Wang, J. (2012). Caenorhabditis elegans RNA-processing protein TDP-1 regulates protein homeostasis and life span. J. Biol. Chem. 287, 8371-8382. Abstract Article

Zhang, W., Hietakangas, V., Wee, S., Lim, S.C., Gunaratne, J., and Cohen, S.M. (2013). ER stress potentiates insulin resistance through PERK-mediated FOXO phosphorylation. Genes Dev. 27, 441-449. Article

Zhang, W., Thompson, B.J., Hietakangas, V., and Cohen, S.M. (2011). MAPK/ERK signaling regulates insulin sensitivity to control glucose metabolism in Drosophila. PLoS Genet. 7, e1002429. Abstract Article

Zhang, Y., Xu, J., Puscau, C., Kim, Y., Wang, X., Alam, H., and Hu, P.J. (2008). Caenorhabditis elegans EAK-3 inhibits dauer arrest via nonautonomous regulation of nuclear DAF-16/FoxO activity. Dev. Biol. 315, 290-302. Abstract Article

All WormBook content, except where otherwise noted, is licensed under a Creative Commons Attribution License. 